TRANSACTIONS OF THE

AMERICAN MATHEMATICAL SOCIETY

Volume 364, Number 6, June 2012, Pages 3071-3135

S 0002-9947(2012)05441-9

Article electronically published on February 3, 2012

\title{
LIE ALGEBROIDS AND CARTAN'S METHOD OF EQUIVALENCE
}

\author{
ANTHONY D. BLAOM
}

\begin{abstract}
Cartan's general equivalence problem is recast in the language of Lie algebroids. The resulting formalism, being coordinate and model-free, allows for a full geometric interpretation of Cartan's method of equivalence via reduction and prolongation. We show how to construct certain normal forms (Cartan algebroids) for objects of finite-type, and are able to interpret these directly as 'infinitesimal symmetries deformed by curvature'.

Details are developed for transitive structures, but rudiments of the theory include intransitive structures (intransitive symmetry deformations). Detailed illustrations include subriemannian contact structures and conformal geometry.
\end{abstract}

c'est la dissymétrie qui crée le phénomène — Pierre Curie, 1894.

\section{Contents}

1. A new setting for Cartan's method of equivalence 3072

2. An outline of Cartan's method à la Lie algebroids 3076

3. Preliminary notions $\quad 3081$

4. Cartan algebroids 3085

5. Examples of infinitesimal geometric structures 3089

6. Generators, associated operators, and Bianchi identities 3096

7. Elementary reduction and image reduction 3101

8. Prolongation and torsion $\quad 3103$

9. $\Theta$-reduction 3106

10. Application: Subriemannian contact three-manifolds 3112

11. Advanced prolongation theory 3117

12. Application: Conformal structures $\quad 3122$

Appendix A. Cartan groupoids and Lie pseudogroups $\quad 3129$

\begin{tabular}{ll|l|}
\hline Appendix B. Miscellany & 3131 \\
\hline
\end{tabular}

References

Received by the editors November 27, 2008 and, in revised form, August 9, 2010.

2010 Mathematics Subject Classification. Primary 53C15, 58H15; Secondary 53B15, 53C07, 53C05, 58H05, 53A55, 53A30, 58A 15.

Key words and phrases. Lie algebroid, Cartan algebroid, equivalence, geometric structure, Cartan geometry, Cartan connection, deformation, differential invariant, pseudogroup, connection theory, G-structure, conformal, prolongation, reduction, subriemannian.

(C)2012 American Mathematical Society Reverts to public domain 28 years from publication 


\section{A neW setting for Cartan's method of Equivalence}

This paper has its origins in attempts to understand, in as invariant a language as possible, Élie Cartan's assertion that finite-type geometric structures are 'symmetries deformed by curvature'. Having identified in [1] a model of geometric structures well-suited to this viewpoint - namely Lie algebroids equipped with a suitably compatible linear connection, called Cartan algebroids - we turn to the problem of realizing this model in practice. To do so requires us to revisit Cartan's method of equivalence, and even to reformulate the basic 'problem of equivalence' that it addresses. The advantages of this reformulation are both theoretical and computational, as we shall demonstrate.

The introduction to this paper is in two parts. In this first section we recast Cartan's equivalence problem in the language of Lie algebroids and formulate specific objectives for the paper. We describe the basic geometric objects with which the paper will be concerned: certain infinitesimal models of geometric structures, and Cartan algebroids, which amount to normal forms thereof.

In Section 2 we outline those elements of Cartan's method which allow us, in the Lie algebroid setting, to associate, with any given object of finite-type, an intrinsically defined Cartan algebroid. Limitations of the method and an outline of the remainder of the paper will also be given there.

1.1. The equivalence problem. Cartan's method of equivalence is a procedure for determining when two objects are equivalent under a change of coordinates. The method applies to an astonishing variety of objects: differential equations, polynomials and variational problems; tensor structures on smooth manifolds, such as Riemannian, conformal, symplectic, complex and almost Kähler structures; differential operators and associated smooth manifold structures, such as affine and projective structures; and so on. For an introduction to the method and a survey of applications see [15, 8, 10, 2, 14].

What makes the method so general is that the equivalence problem is formulated in terms of certain secondary data of universal form, this data encoding essential information about the objects, rather than in terms of the objects themselves. In Cartan's original approach the secondary data is a collection of one-forms (a coframe), defined pointwise up to extra 'group parameters'. In subsequent reformulations, the secondary data is a $G$-structure (see, e.g., [17]) or an exterior differential system (see, e.g., 2]). While in practice the construction of the secondary data can seem ad hoc, it can often be given the following interpretation: it is a geometrization of the PDE's one would write down to find the symmetries (self-equivalences) of the given object, whether or not these symmetries actually exist.

In our Lie algebroid reformulation of Cartan's method, the secondary data here called an infinitesimal geometric structure - can generally be understood as a geometrization of the PDE's one writes down to find the infinitesimal symmetries of the given object. This geometrization is coordinate-free and does not depend on any choice of group, group quotient, or other fixed model. We motivate the formal definition with the following example.

1.2. The 1-symmetries of a Riemannian metric. Consider the problem of finding the infinitesimal symmetries of a Riemannian metric $\sigma$, i.e., its Killing fields, defined on a smooth $n$-dimensional manifold $M$. Call the 1 -jet of a vector field $V$ on $M$, evaluated at some point $m \in M$, a 1-symmetry of $\sigma$, whenever $\sigma$ has, at 
$m \in M$, a vanishing Lie derivative along $V$. Then the collection of all 1-symmetries is a subbundle $\mathfrak{g} \subset J^{1}(T M)$ and $V$ is a Killing field (i.e., genuine symmetry) if and only if its first-order prolongation $J^{1} V$ is a section of $\mathfrak{g}$. The sections of $J^{1}(T M)$ of the form $J^{1} V$ for some $V$ are called holonomic. Whence:

(1) The Killing fields of $\sigma$ are in one-to-one correspondence with the holonomic sections of $\mathfrak{g}$.

Moreover, as we show later, $\sigma$ can be recovered from $\mathfrak{g}$ up to a constant factor, so that little is lost by restricting our attention to $\mathfrak{g}$.

The important observation to make here is that $\mathfrak{g}$ is a Lie algebroid over $M$, as is the tangent bundle $T M$, and its first jet $J^{1}(T M)$. In fact, adopting the natural language associated with these objects, we have:

(2) The bundle $\mathfrak{g} \subset J^{1}(T M)$ of 1-symmetries of a Riemannian metric $\sigma$ is the isotropy subalgebroid of $\sigma$ under the representation of $J^{1}(T M)$ on $\operatorname{Sym}^{2}(T M)$ determined by the adjoint representation of $J^{1}(T M)$ on $T M$.

The terms 'isotropy' and 'adjoint representation' are natural generalizations to Lie algebroids of familiar Lie algebra notions. The adjoint representation of a Lie algebroid is described in in Section 3. Isotropy subalgebroids are defined in Section 5.

A Lie algebroid over a smooth manifold $M$ is a vector bundle $\mathfrak{g}$ on $M$, together with a Lie bracket on its space of sections, and a vector bundle morphism $\#: \mathfrak{g} \rightarrow T M$ is called the anchor, satisfying certain conditions making Lie algebroids generalizations of both tangent bundles (\#:TM $T M$ the identity) and Lie algebras ( $M$ a single point). The bundle of $k$-jets of sections of any Lie algebroid is itself a Lie algebroid.

Lie algebroids over $M$ also generalize the infinitesimal actions of Lie algebras on $M$ (see 1.7 below), in which case the image of the anchor $\#: \mathfrak{g} \rightarrow T M$ is the distribution tangent to the foliation of $M$ by orbits. The image of the anchor of an arbitrary Lie algebroid is always tangent to some foliation, the leaves of which are accordingly called orbits; a Lie algebroid with surjective anchor is called transitive.

1.3. Infinitesimal geometric structures and their symmetries. Infinitesimal geometric structures, as defined below, generalize the vector bundle of 1-symmetries of a Riemannian metric:

Definition. Let $\mathfrak{t}$ be any Lie algebroid over $M$ (the tangent bundle $T M$ in the simplest case). Then an infinitesimal geometric structure on $\mathfrak{t}$ is any subalgebroid $\mathfrak{g} \subset J^{1} \mathfrak{t}$.

In practice, the infinitesimal geometric structure associated with a given object - whether it be a tensor, an operator, or whatever - is constructed as the isotropy subalgebroid of an appropriate Lie algebroid representation. Further examples, both transitive and intransitive, are given in Section 5. Infinitesimal geometric structures are this paper's principal objects of investigation.

The structure kernel of $\mathfrak{g}$ is the kernel $\mathfrak{h} \subset T^{*} M \otimes T M$ of the restriction $a: \mathfrak{g} \rightarrow \mathfrak{t}$ of the projection $J^{1} \mathfrak{t} \rightarrow \mathfrak{t}$, this morphism being the anchor of $\mathfrak{g}$ when $\mathfrak{t}=T M$. This is an analogue of the structure group $G$ of a $G$-structure. The structure kernel of $\mathfrak{g}$ is a subalgebroid whenever it has constant rank.

The image of $\mathfrak{g}$ is the image of $a$. If $a: \mathfrak{g} \rightarrow \mathfrak{t}$ is surjective, we call $\mathfrak{g}$ a surjective infinitesimal geometric structure. If $\mathfrak{t}=T M$, then 'surjective' is synonymous with 
'transitive'. For example, the bundle $\mathfrak{g} \subset J^{1}(T M)$ of 1-symmetries of a Riemannian metric is surjective. We will see that every Poisson structure has an associated infinitesimal geometric structure $\mathfrak{g} \subset J^{1}\left(T^{*} M\right)$ which is surjective but generally not transitive. A simple example of an infinitesimal geometric structure failing to be surjective or transitive is the joint isotropy subalgebroid of a Riemannian metric and a vector field $V$ with non-degenerate energy $\frac{1}{2}\|V\|^{2}$.

Structures sometimes viewed as transitive are actually intransitive, when the notion is invariantly formulated. For example, almost complex structures are generically intransitive structures.

Associated with any $G$-structure on $M$ is a corresponding infinitesimal geometric structure on $T M$, but this structure is always surjective (equivalently, transitive).

Here now, in Lie algebroid language, is an analogue of Cartan's problem of equivalence:

Equivalence Problem. Given smooth manifolds $M_{1}$ and $M_{2}$ and infinitesimal geometric structures $\mathfrak{g}_{1} \subset J^{1}\left(T M_{1}\right)$ and $\mathfrak{g}_{2} \subset J^{1}\left(T M_{2}\right)$, decide whether there exists a diffeomorphism $\phi: M_{1} \rightarrow M_{2}$, with associated tangent map $T \phi: T M_{1} \rightarrow$ $T M_{2}$, such that the corresponding Lie algebroid isomorphism $J^{1}(T \phi): J^{1}\left(T M_{1}\right) \rightarrow$ $J^{1}\left(T M_{2}\right)$ maps $\mathfrak{g}_{1}$ isomorphically onto $\mathfrak{g}_{2}$.

Remark. If we want to formulate a more general equivalence problem, replacing $T M_{1}$ and $T M_{2}$ with general Lie algebroids $\mathfrak{t}_{1}$ and $\mathfrak{t}_{2}$, then we are forced to allow for arbitrary Lie algebroid morphisms $\mathfrak{t}_{1} \rightarrow \mathfrak{t}_{2}$ instead of the tangent maps $T \phi: T M_{1} \rightarrow$ $T M_{2}$, or we must restrict the class of infinitesimal geometric structures considered so that morphisms of 'coordinate change type' can be defined. However, for the restricted purposes of the the present paper, further elaboration on this point will be unnecessary.

1.4. Cartan's method. Having formulated the equivalence problem in terms of appropriate secondary data, Cartan's method attempts to put the data into an appropriate normal form. In the original one-form approach, for example, this normal form is a coframe, on a possibly larger space, but with group parameters eliminated. See, e.g., 8] or [15].

The normalizing algorithm involves two fundamental operations, known as reduction and prolongation. If the secondary data is regarded as a system of PDE's, then reduction amounts to identifying coordinate changes that decouple certain equations from the others, these latter being rendered redundant. Prolongation means increasing the number of variables by introducing derivatives of the independent variables as new independent variables; new equations are added to account for the equality of mixed partial derivatives.

When the normalizing procedure succeeds, the normal form obtained delivers certain basic invariants, and certain 'derived' invariants can be constructed from these. One may test for the equivalence of two objects by comparing the values taken on by the invariants. (Specifically, one compares the associated 'classifying manifold'; see, e.g., [15, Chapter 8].) Objects for which the normalizing procedure succeeds have, at most, finite-dimensional symmetry, and so are called finite-type objects.

The basic invariants of a finite-type object may be understood as obstructions to the existence of a maximal set of symmetries (self-equivalences); they are embodied in the 'curvature' of the associated normal form, when this normal form 
is understood as a 'symmetry deformed by curvature'. In common practice, this interpretation is often obscured, however.

For objects of infinite-type, the normalizing procedure fails to terminate and an altogether different criterion for equivalence must be applied. Infinite-type objects can be identified by applying Cartan's 'involutivity' test and are described extensively in [2, 10. They will not be studied here.

1.5. The symmetries of infinitesimal geometric structures. Analogous to the Killing fields of a Riemannian structure are the symmetries 1 of an infinitesimal geometric structure $\mathfrak{g} \subset J^{1} \mathfrak{t}$. These are those sections $V$ of $\mathfrak{t}$ whose prolongations $J^{1} V \subset J^{1} \mathfrak{t}$ are sections of $\mathfrak{g}$. Evidently, the symmetries of $\mathfrak{g}$ are in one-to-one correspondence with the holonomic sections of $\mathfrak{g} \subset J^{1} \mathfrak{t}$ - those sections that are prolongations of something. Symmetries are necessarily sections of the image of $\mathfrak{g}$ and are closed under the Lie algebroid bracket.

We will not be presenting a complete solution to the equivalence problem here. Rather, our main focus is the following:

Obstruction Problem. Given an infinitesimal geometric structure $\mathfrak{g} \subset J^{1} \mathfrak{t}$, find the obstructions to the existence of symmetries of $\mathfrak{g}$, in the sense defined above.

We now turn our attention to the normal forms that we shall be constructing out of infinitesimal geometric structures. We begin by describing such a normal form explicitly in the case of Riemannian geometry, and by showing concretely how curvature invariants enter as the obstruction to symmetry.

1.6. A normal form for Riemannian geometry. Let $\mathfrak{g} \subset J^{1}(T M)$ be the bundle of 1-symmetries of a Riemannian metric $\sigma$, as described in 1.2 above. Note that the Levi-Cevita connection $\nabla$ associated with $\sigma$ may be regarded as a splitting of a canonical exact sequence,

$$
0 \rightarrow T^{*} M \otimes T M \rightarrow J^{1}(T M) \rightarrow T M \rightarrow 0
$$

We have a corresponding exact sequence,

$$
0 \longrightarrow \mathfrak{h} \longrightarrow \mathfrak{g} \longrightarrow T M \longrightarrow 0,
$$

where $\mathfrak{h} \subset T^{*} M \otimes T M$ denotes the $\mathfrak{o}(n)$-bundle of all $\sigma$-skew-symmetric tangent space endomorphisms, which is $\nabla$-invariant. By the $\nabla$-invariance of $\sigma$, the splitting subbundle of $J^{1}(T M)$ lies inside $\mathfrak{g}$ and we obtain an identification $\mathfrak{g} \cong T M \oplus \mathfrak{h}$. On $\mathfrak{g}$, define a linear connection $\nabla^{(1)}$ by

$$
\nabla_{U}^{(1)}(V \oplus \phi)=\left(\nabla_{U} V+\phi(U)\right) \oplus\left(\nabla_{U} \phi+\operatorname{curv} \nabla(U, V)\right)
$$

Then we will eventually prove that:

(1) A section of $X \subset \mathfrak{g}$ is holonomic if and only if it is $\nabla^{(1)}$-parallel.

In particular, by 1.2(1), the curvature of $\nabla^{(1)}$ is the local obstruction to maximal symmetry, i.e., to the existence, locally, of a set of Killing fields of maximal dimension.

If curv $\nabla^{(1)}=0$, and we assume for simplicity that $M$ is simply connected, then $\mathfrak{g} \cong \mathfrak{g}_{0} \times M$, where $\mathfrak{g}_{0}$ is the $\frac{1}{2} n(n+1)$-dimensional vector space of $\nabla^{(1)}$-parallel sections, which is naturally isomorphic to the Lie algebra of Killing fields.

\footnotetext{
${ }^{1}$ The notions of structure kernel, image, surjectivity, and symmetry all make sense for arbitrary subsets $\mathfrak{g} \subset J^{1} \mathfrak{t}$. No further comment will accompany their application in this extended context.
} 
With the help of the Bianchi identities for linear connections, one computes

$$
\operatorname{curv} \nabla^{(1)}\left(U_{1}, U_{2}\right)(V \oplus \phi)=0 \oplus\left(-\left(\nabla_{V} \operatorname{curv} \nabla+\phi \cdot \operatorname{curv} \nabla\right)\left(U_{1}, U_{2}\right)\right),
$$

implying that $\nabla^{(1)}$ is flat if and only if curv $\nabla$ is both $\nabla$-invariant and $\mathfrak{h}$-invariant. Now $\mathfrak{h}$-invariance implies, by purely algebraic arguments, that curv $\nabla$ has only a scalar component; $\nabla$-invariance then implies constant scalar curvature. Whence from (1) one recovers the standard criterion for maximal local homogeneity of a Riemannian manifold.

1.7. Cartan algebroids: Symmetries deformed by curvature. A linear connection $\nabla$ on a Lie algebroid $\mathfrak{g}$ is a Cartan connection if it is suitably compatible with the Lie algebroid structure [1]. The pair $(\mathfrak{g}, \nabla)$ is then a Cartan algebroid. The formal definition and basic properties are reviewed in Section 4

In the Riemannian example above, the pair $\left(\mathfrak{g}, \nabla^{(1)}\right)$ is a Cartan algebroid, and we saw that $\mathfrak{g} \cong \mathfrak{g}_{0} \times M$, for some Lie algebra $\mathfrak{g}_{0}$, when $\nabla^{(1)}$ is globally flat. In fact, whenever $\mathfrak{g}_{0}$ is any Lie algebra, acting smoothly on a manifold $M$, then the trivial bundle $\mathfrak{g}_{0} \times M$ inherits the structure of a Lie algebroid, called an action (or transformation) algebroid, and the trivial flat connection is a Cartan connection. Conversely, any Cartan algebroid with a flat Cartan connection is locally an action algebroid (Theorem 4.6, Section 4). It is in this sense that Cartan algebroids are infinitesimal symmetries deformed by curvature. Moreover, the orbits of the Cartan algebroid may be regarded as deformations of orbits of a symmetry.

In 1 we described how Cartan algebroids may be viewed as infinitesimal, modelfree, and possibly intransitive versions of classical Cartan geometries, and mentioned other alternative models contained in the literature. Recently, Crampin [6] has delineated the relationship between transitive Cartan algebroids and adjoint tractor bundles [4, which like Cartan algebroids are infinitesimal objects, but unlike them are based on a transitive model fixed a priori.

One consequence of choosing a model-free approach is worth repeating here. Generally, 'curvature' has referred to the local deviation from an underlying model - typically $\mathbb{R}^{n}$ or a homogeneous space $G / H$ - which is fixed. In a model-free approach, such as the one described here, all potential models are created equal, and curvature merely measures the local deviation from some maximally symmetric space. From this point of view, Euclidean space, hyperbolic space, and spheres, are all 'flat' Riemannian manifolds. If Cartan's method determines that a space is flat in the weaker sense, then the particular flat space one has at hand is simply part of the method's output. In model-based approaches model 'mutation' (or something similar) may be needed to detect all possible cases [16.

In Appendix $\mathrm{A}$ we explain how flat Cartan algebroids may be regarded as infinitesimal analogues of Lie pseudogroups of transformations, and discuss the global analogues of arbitrary Cartan algebroids, Cartan groupoids, which may be regarded as 'curved' Lie pseudogroups.

\section{An outline of Cartan's method À la Lie Algebroids}

In this second introductory section we outline the construction of Cartan algebroids from infinitesimal geometric structures. 
2.1. Cartan connections via generators. To solve the obstruction problem formulated in Section 1, we attempt to reduce it to a special case solved in the theorem below. To formulate the result, recall that the linear connections $\nabla$ on a vector bundle $\mathfrak{t}$ are in one-to-one correspondence with the splittings $s: \mathfrak{t} \rightarrow J^{1} \mathfrak{t}$ of a natural exact sequence

$$
0 \rightarrow T^{*} M \otimes \mathfrak{t} \hookrightarrow J^{1} \mathfrak{t} \rightarrow \mathfrak{t} \rightarrow 0 .
$$

Now suppose $\mathfrak{t}$ is a Lie algebroid and $\mathfrak{g} \subset J^{1} \mathfrak{t}$ an infinitesimal geometric structure. Then we call $\nabla$ a generator of $\mathfrak{g} \subset J^{1} \mathfrak{t}$ if $s\left(\mathfrak{t}_{1}\right) \subset \mathfrak{g}$, where $\mathfrak{t}_{1} \subset \mathfrak{t}$ denotes the image of $\mathfrak{g}$. Generators are certain 'preferred connections', which generally exist, but need not be unique. For example, the Levi-Cevita connection is a generator for the bundle $\mathfrak{g} \subset J^{1}(T M)$ of 1-symmetries of a Riemannian metric $\sigma$ (but so is any linear connection $\nabla$ on $M$ such that $\bar{\nabla} \sigma=0$, where $\left.\bar{\nabla}_{U} V:=\nabla_{V} U+[U, V]\right)$. Generators are indispensable in explicit computations.

The following crucial observation is not difficult to establish. (For a proof see Section 6.)

Theorem. Let $\mathfrak{g} \subset J^{1} \mathfrak{t}$ be an infinitesimal geometric structure and assume the projection $a: \mathfrak{g} \rightarrow \mathfrak{t}$ has constant rank. Then $\mathfrak{g}$ has a unique generator $\nabla$ if and only if it is surjective and has structure kernel $\mathfrak{h}=0$. In that case $\nabla$ is a Cartan connection on $\mathfrak{t}$ whose parallel sections are precisely the symmetries of $\mathfrak{g}$.

In particular, curv $\nabla$ is then the local obstruction to maximal symmetry.

When geometric structures do not satisfy the hypotheses of Theorem 2.1, one tries to correct this with an appropriate sequence of prolongation and reduction operations described next.

2.2. Prolongation. The prolongation of an infinitesimal geometric structure $\mathfrak{g} \subset$ $J^{1} \mathfrak{t}$ is a natural 'lift' of $\mathfrak{g}$ to a subset $\mathfrak{g}^{(1)} \subset J^{1} \mathfrak{g}$ : Noting that $J^{1} \mathfrak{g}$ is a subbundle of $J^{1}\left(J^{1} \mathfrak{t}\right)$, and the existence of a natural inclusion $J^{2} \mathfrak{t} \subset J^{1}\left(J^{1} \mathfrak{t}\right)$, we define

$$
\mathfrak{g}^{(1)}:=J^{1} \mathfrak{g} \cap J^{2} \mathfrak{t} .
$$

It turns out that $\mathfrak{g}^{(1)}$ is an infinitesimal geometric structure on $\mathfrak{g}$ whenever $\mathfrak{g}^{(1)}$ has constant rank. Most importantly, there is a one-to-one correspondence between symmetries of $\mathfrak{g}$ and symmetries of $\mathfrak{g}^{(1)}$, furnished by prolongation of sections:

Proposition. A section $W \subset \mathfrak{t}$ is a symmetry of $\mathfrak{g}$ if and only if $J^{1} W \subset \mathfrak{g}$ is a symmetry of $\mathfrak{g}^{(1)}$.

We prove this proposition in Section 8 ,

As an example, consider once again the bundle $\mathfrak{g} \subset J^{1}(T M)$ of 1 -symmetries of a Riemannian metric $\sigma$. Then it is possible to show that $\mathfrak{g}^{(1)} \subset J^{1} \mathfrak{g}$ is surjective with a trivial structure kernel. Replacing $\mathfrak{g}$ and $\mathfrak{t}$ in Theorem 2.1 with $\mathfrak{g}^{(1)}$ and $\mathfrak{g}$, one establishes the existence of the Cartan connection $\nabla^{(1)}$ given explicitly in 1.6 above, as the unique generator of $\mathfrak{g}^{(1)}$. (For details, see 9.6.)

When $\mathfrak{g}$ is transitive one can characterize the prolongation as the joint isotropy of the projection $a: \mathfrak{g} \rightarrow \mathfrak{t}$, viewed as a section $a \subset \mathfrak{g}^{*} \otimes \mathfrak{t}$ (the tautological one-form) and its exterior derivative $d a \subset \operatorname{Alt}^{2}(\mathfrak{g}) \otimes \mathfrak{t}$ (the torsion). This is explained further in Section 8. The isotropy characterization greatly simplifies computations in the transitive case. The isotropy characterization is deduced from general considerations outlined in Section 8 and these should be useful in tackling intransitive cases as well. An in-depth analysis of the transitive case, suitable for making concrete computations, is given in Section 11. 
2.3. Reduction. Let $\mathfrak{g} \subset J^{1} \mathfrak{t}$ be an infinitesimal geometric structure. By a reduction of $\mathfrak{g}$ we shall mean any subalgebroid $\mathfrak{g}^{\prime} \subset \mathfrak{g}$ with the same symmetries as $\mathfrak{g}$; it suffices to check that symmetries of $\mathfrak{g}$ are symmetries of $\mathfrak{g}^{\prime}$. In contrast to prolongation, there is no unique way to construct reductions. Notice, however, that if $\mathfrak{g}^{\prime} \subset \mathfrak{g}$ is a reduction and $\mathfrak{g}^{\prime \prime} \subset \mathfrak{g}$ merely a subalgebroid satisfying $\mathfrak{g}^{\prime} \subset \mathfrak{g}^{\prime \prime} \subset \mathfrak{g}$, then $\mathfrak{g}^{\prime \prime}$ is automatically a reduction of $\mathfrak{g}$ also. We say $\mathfrak{g}^{\prime \prime}$ is a cruder reduction than $\mathfrak{g}^{\prime}$.

We now describe the most important reduction techniques: elementary reduction and $\Theta$-reduction.

2.4. Elementary reduction. Returning to Cartan's method described above, we emphasize that transitivity is not a hypothesis of Theorem 2.1 (and that Cartan algebroids can be intransitive). Rather, one requires surjectivity. If an infinitesimal geometric structure $\mathfrak{g} \subset J^{1} \mathfrak{t}$ is not surjective, we may attempt to make it so by passing to the elementary reduction $\mathfrak{g}_{1}$ of $\mathfrak{g}$. By definition,

$$
\mathfrak{g}_{1}:=\mathfrak{g} \cap J^{1} \mathfrak{t}_{1},
$$

where $\mathfrak{t}_{1} \subset \mathfrak{t}$ denotes the image of $\mathfrak{g}$. Assuming $\mathfrak{t}_{1} \subset \mathfrak{t}$ and $\mathfrak{g}_{1} \subset \mathfrak{g}$ have constant rank, they are subalgebroids. In particular, $\mathfrak{g}_{1} \subset J^{1} \mathfrak{t}_{1}$ becomes an infinitesimal geometric structure. Moreover, one easily proves:

Proposition. If the elementary reduction $\mathfrak{g}_{1}$ of $\mathfrak{g}$ has constant rank, then it is a reduction of $\mathfrak{g}$ in the sense above. If $\mathfrak{g}$ is surjective, then $\mathfrak{g}_{1}=\mathfrak{g}$. Conversely, if $\mathfrak{g}_{1}=\mathfrak{g}$, then $\mathfrak{g}$ is contained in $J^{1} \mathfrak{t}_{1}$ and is surjective, as an infinitesimal geometric structure on $\mathfrak{t}_{1}$.

Because surjectivity is built into the definition of $G$-structures, elementary reduction never appears in that setting. Elementary reduction is described further in Section 7, together with a cruder alternative called image reduction.

2.5. $\Theta$-reduction. If an infinitesimal geometric structure $\mathfrak{g} \subset J^{1} \mathfrak{t}$ is already surjective but has a non-trivial structure kernel, then, with a view to applying Theorem 2.1. one can try to shrink the structure kernel by prolonging (see above). However, the prolongation $\mathfrak{g}^{(1)}$ generally fails to be surjective itself. While one might attempt to correct this by turning to the elementary reduction of $\mathfrak{g}^{(1)}$, there is an alternative that is computationally more attractive. One anticipates the lack of surjectivity of $\mathfrak{g}^{(1)}$ by first replacing $\mathfrak{g}$ by its $\Theta$-reduction. By definition, this is the image of $\mathfrak{g}^{(1)} \subset J^{1} \mathfrak{g}$, i.e., the set $\mathfrak{g}_{1}^{(1)}:=p\left(\mathfrak{g}^{(1)}\right) \subset \mathfrak{g}$, where $p: J^{1} \mathfrak{g} \rightarrow \mathfrak{g}$ is the natural projection. The point is that $\Theta$-reductions can be computed without directly computing the larger space $\mathfrak{g}^{(1)}$. In Section 9 we prove:

Proposition. If $\mathfrak{g}_{1}^{(1)}$ and $\mathfrak{g}^{(1)}$ have constant rank, then the $\Theta$-reduction $\mathfrak{g}_{1}^{(1)}$ of $\mathfrak{g}$ is a reduction of $\mathfrak{g}$ in the sense of [2.3. One has $\mathfrak{g}_{1}^{(1)}=\mathfrak{g}$ if and only if $\mathfrak{g}^{(1)}$ is surjective.

Classically, 'reduction' has usually meant reduction by torsion, a notion we define in 8.5 for arbitrary surjective infinitesimal geometric structures. If $\mathfrak{t}=T M$, then reduction by torsion coincides with $\Theta$-reduction. More generally, however, torsion reduction is a cruder reduction technique. In some cases $\Theta$-reduction is not only more efficient but also more computationally convenient; conformal geometry (Section 12) is a case in point. While $\Theta$-reductions are always defined, we have 
restricted their detailed analysis to the case of surjective structures over transitive Lie algebroids 2 This analysis appears in Section 11.

2.6. A specific normalizing algorithm and its limitations. We now offer a specific algorithm for constructing a Cartan algebroid out of an infinitesimal geometric structure of finite-type. First, we define two auxiliary procedures.

By Proposition 2.4 the following procedure, which we shall call surjectify $\mathfrak{g}$, forces $\mathfrak{g}$ to be surjective:

do while $\mathfrak{g}$ is not surjective end do.

replace $\mathfrak{g}$ with $\mathfrak{g}_{1}$ (elementary reduction)

Next, we let strongly surjectify $\mathfrak{g}$ denote the following procedure making $\mathfrak{g}$ and $\mathfrak{g}^{(1)}$ simultaneously surjective (by Propositions 2.4 and 2.5):

do while $\mathfrak{g}^{(1)}$ is not surjective

surjectify $\mathfrak{g}$

replace $\mathfrak{g}$ with $\mathfrak{g}_{1}^{(1)}$ ( $\Theta$-reduction)

surjectify $\mathfrak{g}$

end do.

To describe an implementation of this procedure it evidently suffices to describe $\Theta$-reduction in the special surjective case.

One might attempt to normalize an infinitesimal geometric structure using elementary reduction and prolongation alone. In practice, however, it is generally easier to apply the following algorithm:

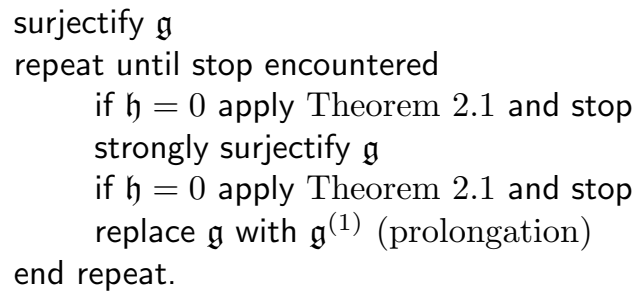

Notice that prolongation is delayed as long as possible. We now describe the ways in which the above algorithm can fail.

First, an execution of surjectify $\mathfrak{g}$ or strongly surjectify $\mathfrak{g}$ could fail because $\mathfrak{g}$, at some iteration of these procedures' do-while loops, loses the constancy of its rank. While prolongation of $\mathfrak{g}$ might resolve this kind of singularity (by recovering rank constancy), this requires a prolongation theory for 'variable rank Lie algebroids' (or Lie pseudoalgebras) which is not provided here. Similarly, $\mathfrak{g}$ might lose rank constancy at some iteration of the repeat-until loop of the main algorithm.

Even if all singularities are successfully resolved, it may happen that no stop is ever encountered in the repeat-until loop, which then becomes perpetual. However, such cases can be detected by applying (a Lie algebroid version of) Cartan's involutivity test; they occur for objects of infinite-type which are not described here.

\footnotetext{
${ }^{2}$ Note added in proof: The transitivity hypothesis in 11.1 and 11.2 can actually be dropped. To see this, work directly with the characterization of $\mathfrak{g}^{(1)}$ based on the maps $\omega_{1}$ and $\omega_{2}$ defined in 8.2 instead of the isotropy characterization discussed above. Details will appear elsewhere.
} 
Another possibility for failure concerns $\Theta$-reduction. In practice, it seems rather complicated to implement without making the added assumption that the base algebroid $\mathfrak{t}$ of $\mathfrak{g} \subset J^{1} \mathfrak{t}$ is transitive. One way to handle intransitivity and singularities might be to restrict, in some way, the infinitesimal geometric structure $\mathfrak{g}$ to each orbit of $\mathfrak{t}$. This restriction will sit over a transitive base (this being a Lie algebroid over the orbit) but is not simply the pullback in the category of Lie algebroids, for one wants an infinitesimal geometric structure over the base, not merely a Lie algebroid. Also, one needs to understand how conclusions regarding the restricted structure combine with transverse information to solve the original problem. Fortunately, a splitting theory for Lie algebroids exists 7 and this possibly reduces the transverse problem to the case of an isolated singularity (zero-dimensional orbit). None of this is explored here either.

If the Cartan algorithm above succeeds it ends in an application of Theorem 2.1, this delivering a Cartan algebroid whose parallel sections are in natural one-to-one correspondence with the symmetries of $\mathfrak{g}$. We then say that $\mathfrak{g}$ admits an associated Cartan algebroid.

2.7. Paper outline. In Section 3 we review basic Lie algebroid notions and establish attendant notation. In particular, we describe the generalizations of linear (Koszul) connections afforded by Lie algebroids, these connections amounting to deformations of Lie algebroid representations, which are also defined. We recall the definition of the adjoint representation of $J^{1} \mathfrak{g}$ on a Lie algebroid $\mathfrak{g}$, and write down the bracket on $J^{1} \mathfrak{g}$ explicitly. We introduce the notion of associated connections, which are ubiquitous throughout, and developed further in Section 6 .

Section 4 summarizes features of Cartan algebroids established in 1, stating, in particular, the result that Cartan algebroids are deformations of infinitesimal symmetries (Theorem 4.6).

Section 5 gives many examples of infinitesimal geometric structures, arising as the isotropy subalgebroids associated with various structures in differential geometry. We also explain how to associate an infinitesimal geometric structure with a Cartan algebroid or a classical $G$-structure. From our description of affine structures, it will be clear how one may associate an infinitesimal geometric structure with an arbitrary (but suitably regular) differential operator on $M$. We demonstrate in practice how one computes the image of an infinitesimal geometric structure, without resorting to local coordinate calculations.

Associated with an infinitesimal geometric structure $\mathfrak{g} \subset J^{1} t$, with structure kernel $\mathfrak{h}$ and image $\mathfrak{t}_{1}$, is an exact sequence

$$
0 \rightarrow \mathfrak{h} \rightarrow \mathfrak{g} \rightarrow \mathfrak{t}_{1} \rightarrow 0
$$

A generator $\nabla$ of $\mathfrak{g}$, as defined in 2.1 above, amounts to a splitting of this sequence, determining an identification $\mathfrak{g} \cong \mathfrak{t}_{1} \oplus \mathfrak{h}$, which is very useful in computations. Section 6 characterizes the linear connections $\nabla$ on $\mathfrak{t}$ arising in this way and writes down the Lie algebroid structure induced on $\mathfrak{t}_{1} \oplus \mathfrak{h}$. Additionally, we express the geometric Bianchi identities in terms of generators and discuss the invariant differential operators associated with a structure. This section can be lightly scanned on a first reading.

Section 7 shows how to explicitly compute an elementary reduction of $\mathfrak{g}$ once a generator is known. A simple application to functions on a Riemannian threemanifold illustrates the technique. 
Sections 8 and 9 are important theoretical parts of the paper, describing prolongation, torsion reduction, and $\Theta$-reduction, and the relationships between these. An immediate application is Theorem 9.4, which furnishes general conditions under which an infinitesimal geometric structure $\mathfrak{g} \subset J^{1} \mathfrak{t}$ is its own associated Cartan algebroid, Riemannian geometry being a case in point. Keen to illustrate the result in this and other concrete examples, we include additional detail in the special case $\mathfrak{t}=T M$ (Theorem 9.5) that is not established until Section 11. The reader preferring a more linear presentation may skip to Section 11 immediately after 9.4 . returning to 9.5 and the remainder of the paper thereafter.

We have not attempted substantially novel applications of Cartan's method in the present work. In particular, our applications to intransitive phenomena are fairly superficial. We hope to correct this deficiency elsewhere. For the purposes of making comparisons with other approaches, we give a detailed application to subriemannian contact three-manifolds in Section 10. An analysis using $G$-structures is to be found in [9, 14]. In addition to constructing obstructions to symmetry, we go on to construct the invariant differential operators.

In Section 11 we return to prolongation, explaining in detail how to 'prolong' a generator, and hence how to compute prolongations in practice. This analysis includes the general case $\mathfrak{t} \neq T M$, but we must assume $\mathfrak{t}$ is transitive to avoid complications. A detailed section on conformal geometry, Section 12, illustrates the more general prolongation results.

\section{PReliminary notions}

For an introduction to Lie groupoids and algebroids, see [3] or [12]. All constructions in this paper are made in the $C^{\infty}$ category.

Notation. We use $\operatorname{Alt}^{k}(V) \cong \Lambda^{k}\left(V^{*}\right)$ and $\operatorname{Sym}^{k}(V) \cong \mathrm{S}^{k}\left(V^{*}\right)$ to denote the spaces of $\mathbb{R}$-valued alternating and symmetric $k$-linear maps on a vector space $V$. Similar notation applies to the tensor algebra of a vector bundle $E$ over $M$. If $\sigma$ is a section of $E$, then this is indicated by writing $\sigma \in \Gamma(E)$ or $\sigma \subset E$. Thus $\sigma \subset \operatorname{Alt}^{2}(T M) \otimes E$ means $\sigma$ is an $E$-valued differential two-form on $M$.

3.1. Lie algebroids. A Lie algebroid over $M$ consists of a vector bundle $\mathfrak{g}$ over $M$, a Lie bracket $[\cdot, \cdot \cdot]$ on the space of sections $\Gamma(\mathfrak{g})$, together with a vector bundle morphism \#: $\mathfrak{g} \rightarrow T M$, called the anchor. One requires that the bracket satisfy a Leibniz identity,

$$
[X, f Y]=f[X, Y]+d f(\# X) Y,
$$

where $f$ is an arbitrary smooth function. The tangent bundle $T M$, equipped with the Jacobi-Lie bracket on vector fields, and identity map as anchor, is a Lie algebroid; in the general case one demands that the induced map of sections $\#: \Gamma(\mathfrak{g}) \rightarrow \Gamma(T M)$ be a Lie algebra homomorphism.

A Lie algebroid is transitive if its anchor is surjective. The image of the anchor is an integrable distribution. The leaves of the integrating foliation, which is possibly singular, are the orbits of the Lie algebroid. A subbundle $\mathfrak{h} \subset \mathfrak{g}$ is a subalgebroid if $\Gamma(\mathfrak{h}) \subset \Gamma(\mathfrak{g})$ is a subalgebra. Lie algebras are simply the Lie algebroids over a single point.

Linear connections on $M$ may be interpreted as representations of the tangent bundle $T M$, deformed by curvature. This point of view leads to the more general 
notion of a $\mathfrak{g}$-connection, defined in 3.3 below. Proposition 3.3 gives an 'analytic' characterization of these connections, generalizing the usual one, which the reader may take as an alternative definition.

3.2. The definition of $\mathfrak{g l}(E)$ for a vector bundle $E$. A representation of a Lie algebra $\mathfrak{g}$ is a vector space $E$, together with a Lie algebra homomorphism $\mathfrak{g} \rightarrow \mathfrak{g l}(E):=\operatorname{Hom}(E, E)$. Now turning to the generalization of $\mathfrak{g l}(E)$ relevant to Lie algebroid representations, let $E$ be a vector bundle over the same base $M$, and consider the exact sequence

$$
0 \rightarrow T^{*} M \otimes E \hookrightarrow J^{1} E \rightarrow E \rightarrow 0
$$

Here

$$
T^{*} M \otimes E \hookrightarrow J^{1} E
$$

is the inclusion which, as a map on sections, send\& $3 d f \otimes \sigma$ to $f J^{1} \sigma-J^{1}(f \sigma)$. Applying $\operatorname{Hom}(\cdot, E)$ to the sequence, and identifying $\operatorname{Hom}\left(T^{*} M \otimes E, E\right)$ with $T M \otimes$ $\operatorname{Hom}(E, E)$, we obtain a second exact sequence

$$
0 \rightarrow \operatorname{Hom}(E, E) \hookrightarrow \operatorname{Hom}\left(J^{1} E, E\right) \stackrel{\#}{\rightarrow} T M \otimes \operatorname{Hom}(E, E) \rightarrow 0 .
$$

Noticing that there is natural inclusion $T M \hookrightarrow T M \otimes \operatorname{Hom}(E, E)$ via the map $v \mapsto v \otimes \mathrm{id}$, we define $\mathfrak{g l}(E) \subset \operatorname{Hom}\left(J^{1} E, E\right)$ to be the preimage of $T M$ under the surjective arrow \#, and obtain a third exact sequence

$$
0 \rightarrow \operatorname{Hom}(E, E) \hookrightarrow \mathfrak{g l}(E) \stackrel{\#}{\rightarrow} T M \rightarrow 0
$$

Proposition. Regard each section $D$ of $\operatorname{Hom}\left(J^{1} E, E\right)$ as a differential operator $D: \Gamma(E) \rightarrow \Gamma(E)$. Then:

(1) A section $D \subset \operatorname{Hom}\left(J^{1} E, E\right)$ lies in $\mathfrak{g l}(E)$ if and only if there exists a vector field $V$ such that

$$
D(f \sigma)=f D \sigma+d f(V) \sigma
$$

for all sections $\sigma$ of $E$ and functions $f$; in that case $V=\# D$.

(2) The operator commutator bracket,

$$
\left[D_{1}, D_{2}\right]_{\mathfrak{g l}(E)} \sigma:=D_{1} D_{2} \sigma-D_{2} D_{1} \sigma
$$

makes $\mathfrak{g l}(E)$ into a Lie algebroid with anchor \#.

Remark. $\mathfrak{g l}(E)$ is in fact a realization of the Lie algebroid of the Lie groupoid $\mathrm{GL}(E)$ of isomorphisms between fibers of the vector bundle $E$, i.e., 'relative frames' on $E$. So elements of $\mathfrak{g l}(E)$ have the interpretation of 'infinitesimal moving frames'. For details, see [12] (where $\mathfrak{g l}(E)$ is denoted $\mathcal{D}(E)$ ).

3.3. Lie algebroid representations and $\mathfrak{g}$-connections. A representation of a Lie algebroid $\mathfrak{g}$ on $E$ is a morphism $\mathfrak{g} \rightarrow \mathfrak{g l}(E)$ of Lie algebroids. When $M$ is a single point one recovers the usual representations of a Lie algebra. Deforming the representation notion we arrive at the following:

Definition. Let $\mathfrak{g}$ be any Lie algebroid over $M$. A $\mathfrak{g}$-connection on a vector bundle $E$ over $M$ is a vector bundle morphism

$$
\nabla: \mathfrak{g} \rightarrow \mathfrak{g l}(E)
$$

\footnotetext{
${ }^{3}$ An opposite sign convention is adopted in our paper [1] and elsewhere. The present convention avoids unpleasant minus signs in 3.6.2) and 3.7)(4).
} 
that is not required to be a Lie algebroid morphism, but is nevertheless required to respect the anchors, $\# \nabla=\nabla \#$.

Suppose $X$ is a section of $\mathfrak{g}$. When the section $\nabla(X)$ of $\mathfrak{g l}(E) \subset \operatorname{Hom}\left(J^{1} E, E\right)$ is to be viewed as a differential operator, we instead write $\nabla_{X}$, i.e., $\nabla_{X} V:=$ $\nabla(X)\left(J^{1} V\right)$. In view of the preceding characterization of the sections of $\mathfrak{g l}(E)$ as derivations, we have the Leibniz identity

$$
\nabla_{X}(f \sigma)=f \nabla_{X} \sigma+d f(\# X) \sigma ; \quad X \subset \mathfrak{g}, \sigma \subset E .
$$

Conversely:

Proposition. Every vector bundle morphism $\nabla: \mathfrak{g} \rightarrow \operatorname{Hom}\left(J^{1} E, E\right)$ that is Leibniz in the above sense is a $\mathfrak{g}$-connection.

If $\nabla$ is a $\mathfrak{g}$-connection, then the formula

$$
\operatorname{curv} \nabla(X, Y):=[\nabla(X), \nabla(Y)]_{\mathfrak{g l}(E)}-\nabla\left([X, Y]_{\mathfrak{g}}\right),
$$

defining the Lie algebroid curvature of the map $\nabla: \mathfrak{g} \rightarrow \mathfrak{g l}(E)$, takes a familiar form:

$$
\operatorname{curv} \nabla(X, Y) Z=\nabla_{X} \nabla_{Y} Z-\nabla_{Y} \nabla_{X} Z-\nabla_{[X, Y]_{\mathfrak{g}}} Z \text {. }
$$

The $\mathfrak{g}$-connection $\nabla$ is a $\mathfrak{g}$-representation when curv $\nabla=0$.

Example. If $\mathfrak{g}$ is a Lie algebroid and $E \subset \mathfrak{g}$ is a subalgebroid contained in the kernel of its anchor, then a canonical representation $\rho$ of $\mathfrak{g}$ on $E$ is well defined by $\rho_{X} Y:=[X, Y]_{\mathfrak{g}}$. Important cases in point are the kernel of the anchor itself, and the structure kernel of an infinitesimal geometric structure, when these have constant rank.

3.4. Linear connections. Using the language of the preceding discussion, a linear connection $\nabla$ on $E$ is just a $T M$-connection on $E$, becoming a $T M$-representation when $\nabla$ is flat. It is an elementary fact that the linear connections on $E$ are in one-to-one correspondence with the splittings $s: E \rightarrow J^{1} E$ of the exact sequence

$$
0 \rightarrow T^{*} M \otimes E \hookrightarrow J^{1} E \rightarrow E \rightarrow 0 .
$$

The splitting associated with a linear connection $\nabla$ on $E$ is, as a map on sections, given by

$$
s \sigma=J^{1} \sigma+\nabla \sigma ; \quad \sigma \subset E .
$$

Here $\nabla \sigma \subset T^{*} M \otimes E$ is defined by $(\nabla \sigma)(V):=\nabla_{V} \sigma$.

3.5. Jet bundles. If $\mathfrak{g}$ is any Lie algebroid over $M$ with anchor \#, then the vector bundle $J^{k} \mathfrak{g}$ of $k$-jets of sections of $\mathfrak{g}$ is another Lie algebroid. Its anchor is the composite

$$
J^{k} \mathfrak{g} \rightarrow \mathfrak{g} \stackrel{\#}{\rightarrow} T M .
$$

Its bracket is uniquely determined by requiring that $k$ th-order prolongation

$$
J^{k}: \Gamma(\mathfrak{g}) \rightarrow \Gamma\left(J^{k} \mathfrak{g}\right)
$$

be a morphism of Lie algebras (but see also 3.7 below). One may view $J^{k}$ as a functor from the category of Lie algebroids to itself $[\mathbf{5}$.

As this paper will demonstrate, many naturally occurring Lie algebroids in differential geometry can be constructed from $T M$ using just two operations: application of the prolongation functor $J^{1}(\cdot)$; and passage to a subalgebroid. 
3.6. The adjoint representation. The generalization of Lie algebra adjoint representations to a Lie algebroid $\mathfrak{g}$ is not a self-representation; it is rather a representation of $J^{1} \mathfrak{g}$ on $\mathfrak{g}$. This representation is well defined by

$$
\operatorname{ad}_{J^{1} X}^{\mathfrak{g}} Y=[X, Y]_{\mathfrak{g}} .
$$

Using the identity

$$
\left[J^{1} X, J^{1} Y\right]_{J^{1} \mathfrak{g}}=J^{1}[X, Y]_{\mathfrak{g}},
$$

one shows that $\operatorname{ad}^{\mathfrak{g}}$ is indeed a representation (and not merely a $J^{1} \mathfrak{g}$-connection).

We note that

$$
\operatorname{ad}_{\phi}^{\mathfrak{g}} X=\phi(\# X) ; \quad X \subset \mathfrak{g},
$$

for all sections $\phi \subset T^{*} M \otimes \mathfrak{g} \subset J^{1} \mathfrak{g}$. If $a: \mathfrak{g} \rightarrow \mathfrak{h}$ is a morphism of Lie algebroids, then one has the identity

$$
a\left(\operatorname{ad}_{\xi}^{\mathfrak{g}} X\right)=\operatorname{ad}_{\left(J^{1} a\right) \xi}^{\mathfrak{h}}(a X) ; \quad \xi \subset J^{1} \mathfrak{g}, X \subset \mathfrak{g} .
$$

3.7. The bracket on $J^{1}(\cdot)$ of a Lie algebroid. Recall that the bracket on $J^{1} \mathfrak{g}$ is implicitly defined by the requirement of [3.6(1). With the help of the adjoint representation, we now describe this bracket concretely.

Although the exact sequence

$$
0 \rightarrow T^{*} M \otimes \mathfrak{g} \hookrightarrow J^{1} \mathfrak{g} \rightarrow \mathfrak{g} \rightarrow 0
$$

possesses no canonical splitting, the corresponding exact sequence of section spaces,

$$
0 \rightarrow \Gamma\left(T^{*} M \otimes \mathfrak{g}\right) \hookrightarrow \Gamma\left(J^{1} \mathfrak{g}\right) \rightarrow \Gamma(\mathfrak{g}) \rightarrow 0,
$$

is split by $J^{1}: \Gamma(\mathfrak{g}) \rightarrow \Gamma\left(J^{1} \mathfrak{g}\right)$, delivering a canonical identification

$$
\Gamma\left(J^{1} \mathfrak{g}\right) \cong \Gamma(\mathfrak{g}) \oplus \Gamma\left(T^{*} M \otimes \mathfrak{g}\right) .
$$

Under this identification, the Lie algebra $\Gamma\left(J^{1} \mathfrak{g}\right)$ is a semidirect product described in the Proposition below.

In addition to having the adjoint representation of $J^{1} \mathfrak{g}$ on $\mathfrak{g}$, we have a representation of $J^{1} \mathfrak{g}$ on $T M$, given by the composite

$$
\begin{gathered}
J^{1} \mathfrak{g} \stackrel{J^{1} \#}{\longrightarrow} J^{1}(T M) \stackrel{\operatorname{ad}^{T M}}{\longrightarrow} \mathfrak{g l}(T M), \\
\text { i.e., } \quad J^{1} X \cdot V=[\# X, V] ; \quad X \subset \mathfrak{g}, V \subset T M .
\end{gathered}
$$

So we can construct a natural representation of $J^{1} \mathfrak{g}$ on $T^{*} M \otimes \mathfrak{g}$; it is given by

$$
\left(J^{1} X \cdot \phi\right) V=[X, \phi(V)]_{\mathfrak{g}}-\phi\left([\# X, V]_{T M}\right) ; \quad V \subset T M .
$$

On the other hand, $T^{*} M \otimes \mathfrak{g}$ is the structure kernel of $J^{1} \mathfrak{g}$ so that $J^{1} \mathfrak{g}$ acts on $T^{*} M \otimes \mathfrak{g}$ via bracket; see the example in 3.3 . A consequence of the following result is that these two representations coincide.

Proposition. The subalgebroid $T^{*} M \otimes \mathfrak{g} \subset J^{1} \mathfrak{g}$ inherits the bracket

$$
\left[\phi_{1}, \phi_{2}\right]_{T^{*} M \otimes \mathfrak{g}}(V)=\phi_{1}\left(\# \phi_{2} V\right)-\phi_{2}\left(\# \phi_{1} V\right) ; \quad V \subset T M,
$$

where \# denotes the anchor of $\mathfrak{g}$. Sections of $J^{1} \mathfrak{g}$ are of the form $J^{1} X+\phi$ for uniquely determined sections $X \subset \mathfrak{g}$ and $\phi \subset T^{*} M \otimes \mathfrak{g}$. The bracket on $J^{1} \mathfrak{g}$ is given by

$$
\left[J^{1} X+\phi, J^{1} Y+\psi\right]_{J^{1} \mathfrak{g}}=J^{1}[X, Y]_{\mathfrak{g}}+J^{1} X \cdot \psi-J^{1} Y \cdot \phi+[\phi, \psi]_{T^{*} M \otimes \mathfrak{g}},
$$

where the action of $J^{1} \mathfrak{g}$ on $T^{*} M \otimes \mathfrak{g}$ is defined by (3). 
To prove the proposition one uses 3.6(1) and the fact that sections of $T^{*} M \otimes \mathfrak{g}$ are finitely generated by those of the form $d f \otimes X=f J^{1} X-J^{1}(f X)$.

3.8. Dual connections, torsion, and associated connections. Let $\mathfrak{g}$ be a Lie algebroid and $\nabla$ a $\mathfrak{g}$-connection on itself. We define the dual of $\nabla$ to be the $\mathfrak{g}$ connection $\nabla^{*}$ on $\mathfrak{g}$ defined by

$$
\nabla_{X}^{*} Y:=\nabla_{Y} X+[X, Y]
$$

One has 'duality' in the sense that $\nabla^{* *}=\nabla$.

The torsion of $\nabla$ is the section, tor $\nabla$, of $\operatorname{Alt}^{2}(\mathfrak{g}) \otimes \mathfrak{g}$ measuring the difference between $\nabla$ and its dual:

$$
\text { tor } \nabla(X, Y):=\nabla_{X} Y-\nabla_{X}^{*} Y=\nabla_{X} Y-\nabla_{Y} X-[X, Y] \text {. }
$$

The torsion or curvature of $\nabla$ can be expressed in terms of the torsion and curvature of $\nabla^{*}$ (and, by duality, vice versa):

Proposition. Let $\nabla$ be a $\mathfrak{g}$-connection on $\mathfrak{g}$, and $\nabla^{*}$ its dual. Then:

$$
\begin{gathered}
\operatorname{tor} \nabla=-\operatorname{tor} \nabla^{*}, \\
\left\{\begin{aligned}
\operatorname{curv} \nabla(X, Y) Z= & \left(\nabla_{Z}^{*} \operatorname{tor} \nabla^{*}\right)(X, Y)-\operatorname{curv} \nabla^{*}(Z, X) Y \\
+ & \operatorname{curv} \nabla^{*}(Z, Y) X ; \quad X, Y, Z \subset \mathfrak{g} .
\end{aligned}\right.
\end{gathered}
$$

We now introduce two important connections generalizing the dual of a linear connection on $T M$. They are examples of associated connections, defined more generally in 6.3

Let $\nabla$ be an arbitrary linear (i.e., $T M$-) connection on a Lie algebroid $\mathfrak{g}$. The associated $\mathfrak{g}$-connection on $\mathfrak{g}$ is defined by

$$
\bar{\nabla}_{X} Y=\nabla_{\# Y} X+[X, Y]_{\mathfrak{g}} ; \quad X, Y \subset \mathfrak{g} .
$$

The associated $\mathfrak{g}$-connection on $T M$ is defined by

$$
\bar{\nabla}_{X} V=\# \nabla_{V} X+[\# X, V]_{T M} ; \quad X \subset \mathfrak{g}, V \subset T M .
$$

The anchor \#: $\mathfrak{g} \rightarrow T M$ is equivariant with respect to these connections: $\bar{\nabla}_{X} \# Y=$ $\# \bar{\nabla}_{X} Y$. If $\mathfrak{g}=T M$ both connections coincide with the dual of $\nabla$.

\section{Cartan algebroids}

This section summarizes some results of 1 where Cartan algebroids were introduced. We also define the cocurvature of an arbitrary linear connection on a Lie algebroid, a notion to reappear in the context of generators of infinitesimal geometric structures. Some further remarks linking flat Cartan algebroids with Lie pseudogroups appear in Appendix A.

As mentioned in Section 1, Cartan algebroids may be regarded as deformations of action algebroids (see Theorem 4.6 below). We recall the definition of the latter first. 
4.1. Action algebroids. Let $\mathfrak{g}_{0}$ be a finite-dimensional Lie algebra with bracket $[\cdot, \cdot]_{\mathfrak{g}_{0}}$ acting smoothly on a manifold $M$. That is, prescribe some Lie algebra homomorphism $\rho: \mathfrak{g}_{0} \rightarrow \Gamma(T M)$. We may regard such actions as abstract infinitesimal symmetries. The trivial bundle $\mathfrak{g}:=\mathfrak{g}_{0} \times M$ over $M$ has a natural Lie algebroid structure. This is the associated action (or transformation) algebroid.

The anchor of this Lie algebroid is the 'action map' \#: $\mathfrak{g}_{0} \times M \rightarrow T M$ sending $(\xi, m)$ to $\rho \xi(m)$. The Lie bracket on $\Gamma\left(\mathfrak{g}_{0} \times M\right)$ is a natural extension of the bracket on $\mathfrak{g}_{0}$, regarded as the subspace $\mathfrak{g}_{0} \subset \Gamma\left(\mathfrak{g}_{0} \times M\right)$ of constant sections. To describe it, let $\tau(\cdot, \cdot)$ denote the naive extension of this bracket, i.e., the one that is bilinear with respect to all smooth functions (and consequently not Leibniz):

$$
\tau(X, Y)(m):=[X(m), Y(m)]_{\mathfrak{g}_{0}} ; \quad X, Y \in \Gamma\left(\mathfrak{g}_{0} \times M\right) .
$$

Also, let $\nabla$ denote the canonical flat connection on $\mathfrak{g}_{0} \times M$. Then the Lie bracket on $\Gamma\left(\mathfrak{g}_{0} \times M\right)$ is defined by

$$
[X, Y]:=\nabla_{\# X} Y-\nabla_{\# Y} X+\tau(X, Y) .
$$

Notice that if $\bar{\nabla}$ denotes the associated $\mathfrak{g}$-connection on $\mathfrak{g}$, then tor $\bar{\nabla}=\tau$.

4.2. Cartan connections. Let $\nabla$ be a linear connection on a Lie algebroid $\mathfrak{g}$. Then $\nabla$ is a Cartan connection if the corresponding splitting

$$
\begin{gathered}
s_{\nabla}: \mathfrak{g} \rightarrow J^{1} \mathfrak{g}, \\
s_{\nabla} \sigma:=J^{1} \sigma+\nabla \sigma
\end{gathered}
$$

of the exact sequence of Lie algebroids,

$$
0 \rightarrow T^{*} M \otimes \mathfrak{g} \hookrightarrow J^{1} \mathfrak{g} \rightarrow \mathfrak{g} \rightarrow 0,
$$

is a Lie algebroid morphism.

A Cartan algebroid is a Lie algebroid equipped with a Cartan connection. A morphism of Cartan algebroids is simply a connection-preserving morphism of the underlying Lie algebroids.

It follows immediately from the definition that the $\mathfrak{g}$-connections $\bar{\nabla}$ on $\mathfrak{g}$ and $T M$, associated with a Cartan connection $\nabla$, are flat, i.e, are $\mathfrak{g}$-representations. In particular, every Cartan algebroid $\mathfrak{g}$ has a canonical self-representation. For a converse statement, see the Corollary below.

4.3. Cocurvature. Associated with an arbitrary linear connection $\nabla$ on $\mathfrak{g}$ is what we call its cocurvature. This is a section of $\operatorname{Alt}^{2}(\mathfrak{g}) \otimes T^{*} M \otimes \mathfrak{g}$, denoted cocurv $\nabla$, measuring the lack of 'compatibility' of $\nabla$ with the Lie algebroid structure of $\mathfrak{g}$ :

$$
\begin{aligned}
\operatorname{cocurv} \nabla(X, Y) V & :=\nabla_{V}[X, Y]-\left[\nabla_{V} X, Y\right]-\left[X, \nabla_{V} Y\right] \\
& +\nabla_{\bar{\nabla}_{X} V} Y-\nabla_{\bar{\nabla}_{Y} V} X .
\end{aligned}
$$

Here $\bar{\nabla}$ denotes the associated $\mathfrak{g}$-connection on $T M$; see 3.8 above. The name 'cocurvature' comes from (5) below.

Define $s_{\nabla}$ as in (11) above. Then the description of the bracket on $J^{1} \mathfrak{g}$ in 3.7 establishes the result (2) below. The other conclusions of the following Proposition are then straightforward.

\section{Proposition.}

(2) $\operatorname{cocurv} \nabla(X, Y)=\operatorname{curv} s_{\nabla}(X, Y):=\left[s_{\nabla} X, s_{\nabla} Y\right]_{J^{1} \mathfrak{g}}-s_{\nabla}[X, Y]_{\mathfrak{g}}$.

(3) $\nabla$ is a Cartan connection if and only if cocurv $\nabla=0$. 
(4) For any sections $X, Y, Z \subset \mathfrak{g}$ and $V \subset T M$ one has

$$
\begin{aligned}
\operatorname{cocurv} \nabla(X, Y) \# Z & =-\operatorname{curv} \bar{\nabla}(X, Y) Z, \\
\# \operatorname{cocurv} \nabla(X, Y) V & =-\operatorname{curv} \bar{\nabla}(\# X, \# Y) V,
\end{aligned}
$$

where $\bar{\nabla}$ denotes the associated $\mathfrak{g}$-connection on $\mathfrak{g}$ in the first formula, and on $T M$ in the second.

(5) In particular, if $\mathfrak{g}=T M$, then

$$
\operatorname{cocurv} \nabla=-\operatorname{curv} \bar{\nabla}
$$

where $\bar{\nabla}$ denotes the dual linear connection on $T M$.

As simple consequences of (4) we have:

\section{Corollary.}

(6) Suppose $\mathfrak{g}$ is transitive. Then $\nabla$ is a Cartan connection on $\mathfrak{g}$ if and only if the associated $\mathfrak{g}$-connection $\bar{\nabla}$ on $\mathfrak{g}$ is flat.

(7) Suppose $\mathfrak{g}$ has an injective anchor. Then $\nabla$ is a Cartan connection if and only if the associated $\mathfrak{g}$-connection $\bar{\nabla}$ on $T M$ is flat.

Although we shall make no use of the fact here, it is worth remarking that a Cartan connection $\nabla$ on a transitive Lie algebroid $\mathfrak{g}$ is uniquely determined by the corresponding self-representation $\bar{\nabla}$; see [1, Proposition 6.1].

4.4. Basic examples of Cartan algebroids. We now list some elementary examples of Cartan algebroids. Example (7) explains the choice of the name 'Cartan algebroid'.

(1) Every action algebroid $\mathfrak{g}_{0} \times M$, equipped with its canonical flat connection $\nabla$, is a Cartan algebroid. Locally this is the only flat example. See 4.6 below.

(2) As we sketch in Appendix $\$$ every Lie pseudogroup of transformations in $M$ has a flat Cartan algebroid as its infinitesimalization.

(3) According to Proposition 4.3(5) a linear connection $\nabla$ on $T M$ is a Cartan connection if and only if its dual $\nabla^{*}$ is flat, i.e., is an infinitesimal parallelism on $M$. By duality, every Cartan connection on $T M$ arises as the dual of some infinitesimal parallelism. See also 5.4.

(4) If $M$ is a Lie group, then the flat linear connection $\nabla$ on $T M$ corresponding to the left (or right) trivialization of $T M$ is, as a special case of (3), a Cartan connection on $T M$.

(5) The distribution $D$ tangent to some regular foliation $\mathcal{F}$ on $M$ is a subalgebroid of $T M$. According to Corollary 4.3(7), a linear connection $\nabla$ on $D$ is a Cartan connection if and only if the $D$-connection $\bar{\nabla}$ on $T M$, defined by $\bar{\nabla}_{U} V=$ $\nabla_{V} U+[U, V], U \subset D, V \subset T M$, is flat.

(6) In 5.8 elementary arguments will show that every torsion-free linear connection $\nabla$ on $T M$ determines a Cartan connection on $J^{1}(T M)$. The parallel sections of this Cartan connection are the prolonged infinitesimal isometries of $\nabla$.

(7) Let $M$ be a classical Cartan geometry modeled on some homogeneous space $G_{0} / H_{0}$ (see, e.g., [16]). If $\pi: P \rightarrow M$ denotes the associated principal $H_{0^{-}}$ bundle, then according to [1], the vector bundle $T P / H_{0}$ is a Lie algebroid supporting a Cartan connection determined by the classical Cartan connection on $P$. (Note, however, that flatness of the former is not sufficient for flatness of the latter; see [1] and our comments on curvature in 1.7]) 
4.5. The symmetric part of a Cartan algebroid. An arbitrary Cartan algebroid $\mathfrak{g}$ has a canonical subalgebroid isomorphic to an action Lie algebroid. Indeed, let $\nabla$ denote the Cartan connection and let $\mathfrak{g}_{0} \subset \Gamma(\mathfrak{g})$ be the subspace of $\nabla$ parallel sections, which is finite-dimensional. Then vanishing cocurvature ensures that $\mathfrak{g}_{0} \subset \Gamma(\mathfrak{g})$ is a Lie subalgebra, and we obtain an action of $\mathfrak{g}_{0}$ on $M$ given by

$$
\begin{gathered}
\mathfrak{g}_{0} \times M \rightarrow T M, \\
(X, m) \mapsto \# X(m) .
\end{gathered}
$$

Equipping the action algebroid $\mathfrak{g}_{0} \times M$ with its canonical flat connection, we obtain a morphism of Cartan algebroids,

$$
\begin{gathered}
\mathfrak{g}_{0} \times M \rightarrow \mathfrak{g}, \\
(X, m) \mapsto X(m) .
\end{gathered}
$$

Assuming $M$ is connected, this morphism is injective because $\nabla$-parallel sections vanishing at a point vanish everywhere. We call the image of the monomorphism (11) the symmetric part of $\mathfrak{g}$.

4.6. Curvature as the local obstruction to symmetry. A Cartan algebroid $\mathfrak{g}$ is globally flat if it is isomorphic to an action algebroid $\mathfrak{g}_{0} \times M$, equipped with its canonical flat connection - or, equivalently, if it coincides with its symmetric part. We call $\mathfrak{g}$ flat if every point of $M$ has an open neighborhood $U$ on which the restriction $\left.\mathfrak{g}\right|_{U}$ is globally flat 4

The following theorem shows that a Cartan algebroid may be viewed as an infinitesimal symmetry deformed by curvature.

Theorem ([1]). Let $\mathfrak{g}$ be a Cartan algebroid with Cartan connection $\nabla$, defined over a connected manifold $M$. Then $\mathfrak{g}$ is flat if and only if curv $\nabla=0$. When $M$ is simply-connected, flatness already implies global flatness.

In the globally flat case the bracket on the Lie algebra $\mathfrak{g}_{0}$ of $\nabla$-parallel sections is given by

$$
[\xi, \eta]_{\mathfrak{g}_{0}}=\operatorname{tor} \bar{\nabla}(\xi, \eta)
$$

where $\bar{\nabla}$ denotes the associated representation of $\mathfrak{g}$ on itself:

$$
\bar{\nabla}_{X} Y=\nabla_{\# Y} X+[X, Y] \text {. }
$$

Proof. The necessity of vanishing curvature is immediate. To establish the assertions in the first paragraph it suffices to show that 4.5)(1) is an isomorphism whenever $\operatorname{curv} \nabla=0$ and $M$ is simply-connected. Indeed, in that case $\nabla$ determines a trivialization of the bundle $\mathfrak{g}$ in which constant sections correspond to the $\nabla$-parallel sections of $\mathfrak{g}$ - that is, to elements of $\mathfrak{g}_{0}$. In particular, $\mathfrak{g}_{0} \times M$ and $\mathfrak{g}$ will have the same rank, implying the monomorphism 4.5(1) is an isomorphism.

The formula (1) holds in the globally flat case because it holds for any action algebroid, as is readily established.

Example. Every Lie group possesses a dual pair of flat linear connections $\nabla, \nabla^{*}$ corresponding to the left and right trivializations of the tangent bundle (see 4.4(4) above). Conversely, whenever a simply-connected manifold $M$ supports a linear connection $\nabla$ on $T M$ such that $\nabla$ and its dual $\nabla^{*}$ are simultaneously flat, then $\nabla$ is a flat Cartan connection on $T M$ and the theorem above delivers an isomorphism

\footnotetext{
${ }^{4}$ In [1] we used symmetric and locally symmetric in place of globally flat and flat, respectively.
} 
$T M \cong \mathfrak{g}_{0} \times M$, where $\mathfrak{g}_{0}$ is the Lie algebra of $\nabla$-parallel vector fields. This isomorphism amounts to a $\mathfrak{g}_{0}$-valued Mauer-Cartan form on $M$, integrating to a Lie group structure under a suitable completeness hypothesis. The Lie bracket on $\mathfrak{g}_{0}$ is given by $[U, V]=-\operatorname{tor} \nabla(U, V)$.

For the application of Theorem 4.6 to examples 4.4(5) and 4.4(7), see [1.

\section{ExAmples of Infinitesimal GeOMEtric STRUCTURES}

In this section we describe the infinitesimal geometric structures associated with Riemannian structures, vector fields on a Riemannian manifold, parallelisms, almost complex structures, Poisson structures, subgeometries of an Abelian Lie group, affine structures, projective structures, classical $G$-structures, and Cartan algebroids.

Subriemannian contact structures and conformal structures are described separately in Sections 10 and 12, Conformal parallelisms are described in 9.7

5.1. Isotropy. Most infinitesimal geometric structures occurring in nature are best understood as isotropy (or joint isotropy) subalgebroids, of certain jet-bundle representations. In the case of Riemannian geometry it is the isotropy of the Riemannian metric $\sigma \subset \operatorname{Sym}^{2}(T M)$, i.e., of a section of some vector bundle; in conformal geometry, it is the isotropy of a rank-one subbundle $\langle\sigma\rangle \subset \operatorname{Sym}^{2}(T M)$; in projective geometry, it is the isotropy of an affine subbundle of $J^{1}(T M)^{*} \otimes T^{*} M \otimes T M$. The following definition of isotropy is general enough to cover all these possibilities.

Let $\rho: \mathfrak{g} \rightarrow \mathfrak{g l}(E)$ denote some representation of a Lie algebroid $\mathfrak{g}$. Let $\Sigma \subset E$ denote any affine subbundle of $E$ (a single section of $E$ in the simplest case) and $\Sigma_{0} \subset E$ the corresponding vector subbundle parallel to $\Sigma$ (resp., the zero section). Assume that either $\Sigma_{0}$ is $\mathfrak{g}$-invariant or $\Sigma=\Sigma_{0}$. Then the isotropy of $\Sigma$ is the collection of all elements $x \in \mathfrak{g}$ for which

$$
\sigma \subset \Sigma \Longrightarrow \rho_{x} \sigma \in \Sigma_{0},
$$

for arbitrary local sections $\sigma \subset E$; here $\rho_{x} \sigma:=\rho(x)\left(J^{1} \sigma(m)\right)$, where $m \in M$ is the base point of $x$.

The isotropy of $\Sigma$ is a subset of $\mathfrak{g}$ intersecting fibers in subspaces whose dimensions may vary, i.e., is a 'variable-rank subbundle'; sections of this bundle are closed under the bracket of $\mathfrak{g}$. When this rank is constant the isotropy is a bona fide subbundle and consequently a subalgebroid, called the isotropy subalgebroid of $\Sigma$. A section $X$ of $\mathfrak{g}$ is then a section of the isotropy if and only if $\rho_{X} \sigma$ is a section of $\Sigma_{0}$ for all sections $\sigma \subset \Sigma$.

5.2. Riemannian structures. The adjoint representation of $J^{1}(T M)$ on $T M$ determines a representation of $J^{1}(T M)$ on $\operatorname{Sym}^{2}(T M)$ : a section $X \subset J^{1}(T M)$ acts on a section $\sigma \subset \operatorname{Sym}^{2}(T M)$ according to

$$
(X \cdot \sigma)\left(V_{1}, V_{2}\right):=\mathcal{L}_{\# X}\left(\sigma\left(V_{1}, V_{2}\right)\right)-\sigma\left(\operatorname{ad}_{X}^{T M} V_{1}, V_{2}\right)-\sigma\left(V_{1}, \operatorname{ad}_{X}^{T M} V_{2}\right) .
$$

Since this simply means $J^{1} V \cdot \sigma=\mathcal{L}_{V} \sigma$, the isotropy $\mathfrak{g} \subset J^{1}(T M)$ of a Riemannian metric $\sigma \subset \operatorname{Sym}^{2}(T M)$ is indeed its associated bundle of 1-symmetries, as described in 1.2

According to (1), the subalgebroid $T^{*} M \otimes T M \subset J^{1}(T M)$ acts on $\operatorname{Sym}^{2}(T M)$ via

$$
(\phi \cdot \sigma)\left(V_{1}, V_{2}\right)=-\sigma\left(\phi V_{1}, V_{2}\right)-\sigma\left(V_{1}, \phi V_{2}\right) ; \quad \phi \subset T^{*} M \otimes T M .
$$


The structure kernel of $\mathfrak{g}$ is the isotropy $\mathfrak{h} \subset T^{*} M \otimes T M$ of $\sigma$ under this restricted representation. So $\mathfrak{h}$ is the bundle of $\sigma$-skew-symmetric endomorphisms of tangent spaces, a Lie algebra bundle modeled on $\mathfrak{o}(n), n:=\operatorname{dim} M$. In particular, all conformally equivalent metrics give the same structure kernel.

One way to see that $\mathfrak{g}$ is surjective (i.e., transitive) is to apply the algebraic lemma B.1 in Appendix B to the morphism $X \mapsto X \cdot \sigma: J^{1}(T M) \rightarrow \operatorname{Sym}^{2}(T M)$, whose kernel is $\mathfrak{g}$. Due to the surjectivity of the restriction $\phi \mapsto \phi \cdot \sigma: T^{*} M \otimes T M \rightarrow$ $\operatorname{Sym}^{2}(T M)$ of this morphism, the lemma delivers an exact sequence

$$
0 \rightarrow \mathfrak{h} \rightarrow \mathfrak{g} \rightarrow T M \rightarrow 0 .
$$

Thus $\mathfrak{g} \subset J^{1}(T M)$ is surjective and has constant rank (making it a subalgebroid and thus an infinitesimal geometric structure).

The lemma just applied is very useful in determining the image and structure kernel of infinitesimal geometric structures defined by isotropy. Less trivial applications of Lemma B.1 are made in 5.3 and 5.5 below. Minimal comment will accompany subsequent applications.

The symmetries of $\mathfrak{g}$ (in the sense of 1.3) are the vector fields along which $\sigma$ has vanishing Lie derivative, i.e., its Killing fields. A generator of $\mathfrak{g}$ (see 2.1) is a linear connection $\nabla$ on $T M$ such that $\sigma$ is $\bar{\nabla}$-parallel, where $\bar{\nabla}$ denotes the dual of $\nabla$. The Levi-Cevita connection is thus the unique torsion-free generator of $\mathfrak{g}$.

From $\mathfrak{g}$ one can recover the metric $\sigma$ up to a positive constant (not merely its conformal class). In the simply-connected case, slightly more is true:

Proposition. Let $\mathfrak{h} \subset T^{*} M \otimes T M$ denote the $\mathfrak{o}(n)$-bundle associated with an arbitrary conformal structure. Then on simply-connected open subsets of $M$, every surjective infinitesimal geometric structure $\mathfrak{g} \subset J^{1}(T M)$ having structure kernel $\mathfrak{h}$ is the isotropy subalgebroid of some Riemannian structure $\sigma$ in the given conformal class. This structure is uniquely determined up to a constant.

Proof. Suppose $\mathfrak{g} \subset J^{1}(T M)$ has structure kernel $\mathfrak{h}$ and let $L \subset \operatorname{Sym}^{2}(T M)$ be the line bundle determined by the conformal structure. That is, $L$ is the bundle of $\mathfrak{h}$-invariant elements of $\operatorname{Sym}^{2}(T M)$. The non-vanishing elements of $L$ are either positive or negative definite. By Lemma B.2 $L$ has a non-vanishing $\mathfrak{g}$-invariant section $\sigma$, unique up to constant. Changing the sign of $\sigma$ if necessary, we obtain the sought after metric.

The application of Cartan's method to Riemannian structures is given in 9.6

In analogy with the Riemannian case, the isotropy subset $\mathfrak{g} \subset J^{1}(T M)$ of an arbitrary tensor on $M$ is an infinitesimal geometric structure, whenever this isotropy has constant rank. Moreover, in many cases, this structure encodes all useful information (i.e., some analogue of the preceding proposition applies).

For structures defined by more than one tensor one considers the joint isotropy, defined by intersecting the individual isotropies. For example, for almost Kähler structures, one considers the joint isotropy of the symplectic and almost complex structures. Here is another example:

5.3. Vector fields on a Riemannian manifold. Let $V$ be a nowhere vanishing vector field on a Riemannian manifold $M$ with metric $\sigma$. The vector fields on $M$ that are simultaneously infinitesimal isometries of $\sigma \subset \operatorname{Sym}^{2}(T M)$ and $V \subset T M$ are the symmetries of the joint isotropy of $\sigma$ and $V$, with respect to representations of $J^{1}(T M)$ on $\operatorname{Sym}^{2}(T M)$ and $T M$, respectively. Denoting the isotropy of $\sigma$ alone 
by $\mathfrak{g} \subset J^{1}(T M)$ as above, $\mathfrak{g}$ acts on $T M$ by restricted adjoint action and the joint isotropy is the isotropy $\mathfrak{g}_{V} \subset \mathfrak{g}$ of $V$.

The structure kernel of $\mathfrak{g}_{V}$ is the $\mathfrak{o}(n-1)$-bundle of skew-symmetric tangent space endomorphisms infinitesimally fixing $V$ (mapping $V$ to 0 ).

Proposition. The image of $\mathfrak{g}_{V}$ is the distribution $D \subset T M$ tangent to the level sets of $\frac{1}{2}\|V\|^{2}$.

In particular, $\mathfrak{g}$ has constant rank (is an infinitesimal geometric structure) if and only if $V$ has constant length or $\frac{1}{2}\|V\|^{2}$ is free of critical points; $\mathfrak{g}_{V}$ is surjective (transitive) in the former case only.

Proof. Applying Lemma B.1 to the morphism $X \mapsto X \cdot V: \mathfrak{g} \rightarrow T M$, of which $\mathfrak{g}_{V}$ is the kernel, we deduce that $D$ is the kernel of the morphism $\Theta$ making the following diagram commute:

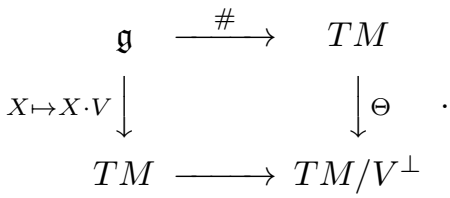

Let $\nabla$ be any generator of $\mathfrak{g}$ (e.g., the Levi-Cevita connection) and $s: T M \rightarrow$ $\mathfrak{g}$ the corresponding splitting of (2) above. Then $\Theta(U)=s U \cdot V \bmod V^{\perp}=$ $\bar{\nabla}_{U} V \bmod V^{\perp}$, where $\bar{\nabla}$ is the dual connection. Or, identifying $T M / V^{\perp}$ with the trivial line bundle $\mathbb{R} \times M$, using $V$, we have $\Theta(U)=\sigma\left(V, \bar{\nabla}_{U} V\right)=\frac{1}{2} \mathcal{L}_{U}(\sigma(V, V))=$ $d\left(\frac{1}{2}\|V\|^{2}\right)(U)$. Here we have used $\bar{\nabla} \sigma=0$, which holds because $\nabla$ is a generator.

5.4. Parallelism. The simplest non-trivial example of an infinitesimal geometric structure is a transitive infinitesimal geometric structure $\mathfrak{g} \subset J^{1}(T M)$ having a trivial structure kernel. In other words, $\mathfrak{g}$ is a subalgebroid of $J^{1}(T M)$ mapped isomorphically onto $T M$ by the anchor $\#: J^{1}(T M) \rightarrow T M$. According to Theorem 2.1. $\mathfrak{g}$ has a unique generator $\nabla$ that is a Cartan connection on $T M$. From our observations in 4.4(3), the dual connection $\bar{\nabla}$ is flat, i.e., an infinitesimal parallelism on $M$, and conversely all infinitesimal parallelisms arise in this way.

When $M$ is simply-connected the Lie algebroid morphism $\bar{\nabla}: T M \rightarrow \mathfrak{g l}(T M)$ integrates to a Lie groupoid morphism $M \times M \rightarrow \mathrm{GL}(T M)$, i.e., to an absolute parallelism on $M$ (a trivialization of the tangent bundle).

When $\bar{\nabla}$ comes from an absolute parallelism, viewed instead as some nondegenerate $V$-valued 1-form $\omega$ on $M(\operatorname{dim} V=\operatorname{dim} M)$, then $\mathfrak{g} \subset J^{1}(T M)$ is the isotropy subalgebroid of $\omega \subset T^{*} M \otimes V$, and a symmetry of $\mathfrak{g}$ is a vector field along which $\omega$ has vanishing Lie derivative.

The infinitesimal geometric structure associated with a conformal parallelism is described in 9.7

5.5. Almost complex structures. The infinitesimal geometric structure associated with an almost complex structure $\mathbf{J}$ on $M$ is generically intransitive. Indeed, let $\mathfrak{g} \subset J^{1}(T M)$ denote the isotropy of $\mathbf{J} \subset T^{*} M \otimes T M$ under the representation of $J^{1}(T M)$ on $T^{*} M \otimes T M$ induced by the adjoint representation, and define

$$
\left[T^{*} M \otimes T M, \mathbf{J}\right]:=\bigcup_{m \in M}\left\{\phi \mathbf{J}(m)-\mathbf{J}(m) \phi \mid \phi \in T_{m}^{*} M \otimes T_{m} M\right\} ;
$$


let $N \subset \operatorname{Alt}^{2}(T M) \otimes T M$ denote the Nijenhuis torsion of $\mathbf{J}$ :

$$
N(U, V)=\frac{1}{4}([\mathbf{J} U, \mathbf{J} V]-[U, V]-\mathbf{J}[U, \mathbf{J} V]-\mathbf{J}[\mathbf{J} U, V]) .
$$

Then:

Proposition. The structure kernel of $\mathfrak{g}$ is $T^{*} M \otimes_{\mathbb{C}} T M$ and the image of $\mathfrak{g}$ is the kernel of the morphism

$$
\begin{gathered}
\Theta: T M \rightarrow\left(T^{*} M \otimes T M\right) /\left[T^{*} M \otimes T M, \mathbf{J}\right], \\
\Theta(U)=-4 N(\mathbf{J} U, \cdot,) \bmod \left[T^{*} M \otimes T M, \mathbf{J}\right] .
\end{gathered}
$$

In particular, $\mathfrak{g}$ is transitive if and only if the section $N(U, \cdot) \subset T^{*} M \otimes T M$ lies in $\left[T^{*} M \otimes T M, \mathbf{J}\right]$ for all vector fields $U$.

Proof. First, note that

$$
\frac{1}{2}\left[\operatorname{ad}_{J^{1}(\mathbf{J} U)} \mathbf{J}, \mathbf{J}\right] V=-\mathbf{J}[\mathbf{J} U, \mathbf{J} V]-[\mathbf{J} U, V] ; \quad U, V \subset T M .
$$

Next observe that $\mathfrak{g}$ is the kernel of the morphism

$$
\begin{gathered}
\theta: J^{1}(T M) \rightarrow T^{*} M \otimes T M \\
\theta(X)=\operatorname{ad}_{X} \mathbf{J}, \\
\text { i.e., } \quad \theta(X) U=\operatorname{ad}_{X}(\mathbf{J} U)-\mathbf{J}\left(\operatorname{ad}_{X} U\right) .
\end{gathered}
$$

Applying Lemma B.1 to this morphism, we obtain a morphism $\Theta$ with the requisite kernel, and satisfying

$$
\begin{aligned}
\Theta(U) & =\operatorname{ad}_{J^{1} U} \mathbf{J} \bmod \left[T^{*} M \otimes T M, \mathbf{J}\right] \\
& =\operatorname{ad}_{J^{1} U} \mathbf{J}-\frac{1}{2}\left[\operatorname{ad}_{J^{1}(\mathbf{J} U)} \mathbf{J}, \mathbf{J}\right] \bmod \left[T^{*} M \otimes T M, \mathbf{J}\right] .
\end{aligned}
$$

By (11), we have

$$
\begin{aligned}
\left(\operatorname{ad}_{J^{1} U} \mathbf{J}-\frac{1}{2}\left[\operatorname{ad}_{J^{1}(\mathbf{J} U)} \mathbf{J}, \mathbf{J}\right]\right) V=[ & U, \mathbf{J} V]-\mathbf{J}[U, V] \\
& +\mathbf{J}[\mathbf{J} U, \mathbf{J} V]+[\mathbf{J} U, V]=-4 N(\mathbf{J} U, V) .
\end{aligned}
$$

5.6. Poisson structures. Although not of finite-type, Poisson structures furnish us with another interesting example of an infinitesimal geometric structure. Now generally the isotropy $\mathfrak{g} \subset J^{1}(T M)$ of a Poisson tensor on $M$ fails to have constant rank, and so fails to be an infinitesimal geometric structure on $T M$. However, one can define an infinitesimal geometric structure on the cotangent bundle $T^{*} M$, which the Poisson tensor makes into a Lie algebroid (see below). Although not transitive, this structure is surjective.

Let $\omega$ be a symplectic structure on $M$ and let $\#: T^{*} M \rightarrow T M$ denote the inverse of $v \mapsto \omega(v, \cdot)$. Since \# is an isomorphism, there is a unique bracket on $\Gamma\left(T^{*} M\right)$ making $T^{*} M$ into a Lie algebroid with anchor \#. This bracket is given by

$$
[\alpha, \beta]_{T^{*} M}=\mathcal{L}_{\# \alpha} \beta-\mathcal{L}_{\# \beta} \alpha+d(\Pi(\alpha, \beta)), \quad \alpha, \beta \in \Gamma\left(T^{*} M\right),
$$

where $\mathcal{L}$ denotes Lie derivative and $\Pi$ is the Poisson tensor. This tensor is defined by $\Pi(\alpha, \beta):=\omega(\# \alpha, \# \beta)$ and so satisfies

$$
\langle\alpha, \# \beta\rangle=\Pi(\alpha, \beta) \quad \alpha, \beta \in \Gamma\left(T^{*} M\right) .
$$


More generally, (11) defines a Lie algebroid structure on $T^{*} M$ for any Poisson manifold $(M, \Pi)$, with anchor \# defined by (2). The symplectic leaves of $\Pi$ are precisely the orbits of the Lie algebroid $T^{*} M$.

An infinitesimal isometry of a Poisson manifold $(M, \Pi)$ is a vector field $V$ on $M$ such that $\mathcal{L}_{V} \Pi=0$. Poisson manifolds have an abundance of infinitesimal isometries. In particular, every closed 1-form $\alpha$ on $M$ determines an infinitesimal isometry $\# \alpha$ tangent to the symplectic leaves known as a local Hamiltonian vector field, or a Hamiltonian vector field if $\alpha$ is exact.

It is not too difficult to establish the following result; see [1] for some details:

Proposition. Let $\mathfrak{g} \subset J^{1}\left(T^{*} M\right)$ denote the kernel of the vector bundle morphism $J^{1}\left(T^{*} M\right) \rightarrow \operatorname{Alt}^{2}(T M)$ whose corresponding map on sections sends $J^{1} \alpha$ to $d \alpha$. Then $\mathfrak{g}$ is a surjective infinitesimal geometric structure on $T^{*} M$, with structure kernel $\operatorname{Sym}^{2}(T M)$, whose symmetries are the closed one-forms on $M$.

$A$ linear connection $\nabla$ on $T^{*} M$ is a generator of $\mathfrak{g}$ if and only if the corresponding linear connection on TM is torsion-free. Such a generator is a Cartan connection on $T^{*} M$ if and only if

$$
\operatorname{curv} \nabla(V, \# \alpha) \beta-\operatorname{curv} \nabla(V, \# \beta) \alpha-\left(\nabla_{V}(\nabla \Pi)\right)(\alpha, \beta)=0,
$$

for all sections $\alpha, \beta \subset T^{*} M ; V \subset T M$.

If $M$ is the dual of a Lie algebra, equipped with its Lie-Poisson structure (see, e.g., [13, §10.1]), then the canonical flat linear connection $\nabla$ on $T^{*} M \cong M \times M^{*}$ is an example of a Cartan connection as described in the Proposition. Up to certain momentum map equivariance obstructions, this is locally the only flat example [1, Corollary 3.4].

5.7. Subgeometries of an Abelian Lie group. We now give a simple example of a geometric structure which has, in general, trivial isotropy. Let $E$ be an Abelian Lie group, $V$ its Lie algebra, and $M \subset E$ a codimension-one submanifold. Let $\omega$ be the $V$-valued one-form on $M$ obtained by restricting the Mauer-Cartan form on $E$. Then $d \omega=0$ and $\operatorname{dim} V=\operatorname{dim} M+1$.

Let $\omega(T M)$ denote the tangent bundle of $\mathrm{M}$, viewed as a subbundle of $V \times M$, so that $N:=(V \times M) / \omega(T M)$ is a model of the rank-one normal bundle of $M$ in $E$. A basic invariant of $M$ associated with its embedding into $E$ is the quadratic form $\mathrm{II} \subset \operatorname{Sym}^{2}(T M) \otimes N$ defined by

$$
\mathbb{I}\left(U_{1}, U_{2}\right):=(\nabla \omega)_{\mathrm{sym}}\left(U_{1}, U_{2}\right) \bmod \omega(T M),
$$

where $(\nabla \omega)_{\text {sym }}\left(U_{1}, U_{2}\right):=\left(\nabla_{U_{1}} \omega\right) U_{2}+\left(\nabla_{U_{2}} \omega\right) U_{1}$. Here $\nabla$ is an arbitrary linear connection on $M$, the particular choice not effecting the definition of II.

Proposition. The isotropy $\mathfrak{g} \subset J^{1}(T M)$ of $\omega$ has a trivial structure kernel, and has image ker II. In particular, if the quadratic form $\mathbb{I I}$ is non-degenerate, then $\mathfrak{g}=0$ and $\omega$ has no infinitesimal isometries.

Proof. Apply Lemma B.1 to the morphism $\theta: J^{1}(T M) \rightarrow T^{*} M \otimes V ; \theta(X):=X \cdot \omega$. Here $(X \cdot \omega)(U)=\mathcal{L}_{\# X}(\omega(U))-\omega\left(\operatorname{ad}_{X} U\right)$. Then the morphism $\Theta$ in that lemma 
is given by

$$
\begin{aligned}
\Theta(U) & =\mathcal{L}_{U} \omega \bmod \omega(T M) \\
& =\frac{1}{2}(\nabla \omega)_{\mathrm{sym}}(U, \cdot) \bmod \omega(T M), \text { because of the identity } \\
\left(\mathcal{L}_{U_{1}} \omega\right)\left(U_{2}\right) & =\omega\left(\nabla_{U_{2}} U_{1}\right)+\frac{1}{2} d \omega\left(U_{1}, U_{2}\right)+\frac{1}{2}(\nabla \omega)_{\mathrm{sym}}\left(U_{1}, U_{2}\right) .
\end{aligned}
$$

5.8. Affine structures. Any suitably non-degenerate, $k$ th-order, linear, differential operator on $M$ defines an infinitesimal geometric structure $\mathfrak{g} \subset J^{k+1}(T M) \subset$ $J^{1}\left(J^{k}(T M)\right)$. As a simple example, which will suffice to illustrate the general principle, we consider an affine structure on $M$, i.e., an arbitrary linear connection $\nabla$ on $M$, in which case $k=1$. The relevant non-degeneracy condition is that the isotropy of the torsion of $\nabla$ should have constant rank; see below.

View an affine structure $\nabla$ as a section of $J^{1}(T M)^{*} \otimes T^{*} M \otimes T M$, via

$$
\nabla\left(J^{1} W, V\right):=\nabla_{V} W ; \quad V, W \subset T M .
$$

In order to associate a natural isotropy subalgebroid with $\nabla$, we begin with two observations. First, $J^{1}\left(J^{1}(T M)\right)$ acts on $J^{1}(T M)^{*} \otimes T^{*} M \otimes T M$ because $J^{1}\left(J^{1}(T M)\right)$ acts on $J^{1}(T M)$ via adjoint action, and on $T M$ via the composite

$$
\begin{gathered}
J^{1}\left(J^{1}(T M)\right) \stackrel{p}{\rightarrow} J^{1}(T M) \stackrel{\operatorname{ad}^{T M}}{\longrightarrow} \mathfrak{g r}(T M), \\
\text { i.e., } \quad J^{1} X \cdot W=\operatorname{ad}_{p X}^{T M} W ; \quad X \subset J^{1}(T M), W \subset T M .
\end{gathered}
$$

Secondly, $J^{2}(T M)$ may be identified with a subalgebroid of $J^{1}\left(J^{1}(T M)\right)$ via the canonical embedding $J^{2}(T M) \hookrightarrow J^{1}\left(J^{1}(T M)\right)$ whose corresponding map on sections sends $J^{2} V$ to $J^{1}\left(J^{1} V\right)$. Combining the two observations, we obtain a natural action of $J^{2}(T M)$ on $J^{1}(T M)^{*} \otimes T^{*} M \otimes T M$.

Proposition. Let $\mathfrak{g} \subset J^{2}(T M)$ denote the isotropy of $\nabla \subset J^{1}(T M)^{*} \otimes T^{*} M \otimes T M$, and $\mathfrak{t} \subset J^{1}(T M)$ the isotropy of tor $\nabla \subset \operatorname{Alt}^{2}(T M) \otimes T M$. Then:

(1) The symmetries of $\mathfrak{g}$ are the prolonged infinitesimal isometries of $\nabla$.

(2) The image of $\mathfrak{g} \subset J^{1}\left(J^{1}(T M)\right)$ is $\mathfrak{t}$, and $\mathfrak{g}$ has a trivial structure kernel.

In particular, (2) implies that $\mathfrak{g} \subset J^{2}(T M)$ has constant rank (and is therefore an infinitesimal geometric structure on $J^{1}(T M)$ ) if and only if $\mathfrak{t} \subset J^{1}(T M)$ has constant rank.

Now $\mathfrak{t}=J^{1}(T M)$ if and only if $\nabla$ is torsion-free (because $\operatorname{id}_{T M}$ is a section of $\left.T^{*} M \otimes T M \subset J^{1}(T M)\right)$. Due to (2), $\mathfrak{g}$ is surjective if and only if $\nabla$ is torsion-free. Applying Theorem 2.1, we obtain:

Corollary. If tor $\nabla=0$, then the unique generator $\nabla^{(1)}$ of $\mathfrak{g}$ is a Cartan connection on $J^{1}(T M)$ whose parallel sections are the prolonged infinitesimal isometries of $\nabla$.

From an explicit formula for $\nabla^{(1)}$ one may completely characterize the obstructions to the existence of infinitesimal isometries; see 11.5.

Proof of the Proposition. Recall that a vector field $U$ on $M$ is an infinitesimal isometry of $\nabla$ if

$$
\left[U, \nabla_{V} W\right]-\nabla_{[U, V]} W-\nabla_{V}[U, W]=0 ; \quad V, W \subset T M,
$$


a condition that is second-order in $U$. Unravelling the definition of the representations defined above, we may write this condition as

$$
J^{1}\left(J^{1}(U)\right) \cdot \nabla=0 .
$$

It easily follows that $J^{1} U$ is a symmetry of $\mathfrak{g}$ whenever $U$ is an infinitesimal isometry of $\nabla$.

Suppose, conversely, that $X \subset J^{1}(T M)$ is a symmetry of $\mathfrak{g}$, i.e., that $J^{1} X$ lies in $\mathfrak{g}$. This means

$$
\begin{aligned}
& J^{1} X \subset J^{2}(T M) \\
& \text { and } J^{1} X \cdot \nabla=0 .
\end{aligned}
$$

It is well known that (4) is equivalent to $X \subset J^{1}(T M)$ being holonomic (see Lemma 8.1). So $X=J^{1} U$, where $U$ is an infinitesimal isometry, due to (5), which reads $J^{1}\left(J^{1} U\right) \cdot \nabla=0$. This completes the proof of (1).

Let $\xi$ be any section of $J^{2}(T M)$. It is easy to check that the section $\xi \cdot \nabla \subset$ $J^{1}(T M)^{*} \otimes T^{*} M \otimes T M$ is tensorial, i.e., drops to some section $(\xi \cdot \nabla)^{\vee} \subset T^{*} M \otimes$ $T^{*} M \otimes T M$. Noting that $\mathfrak{g}$ is then the kernel of the morphism

$$
\begin{aligned}
\xi & \mapsto(\xi \cdot \nabla)^{\vee}, \\
J^{2}(T M) & \rightarrow T^{*} M \otimes T^{*} M \otimes T M,
\end{aligned}
$$

whose domain $J^{2}(T M)$ fits into an exact sequence

$$
0 \rightarrow \operatorname{Sym}^{2}(T M) \otimes T M \hookrightarrow J^{2}(T M) \rightarrow J^{1}(T M) \rightarrow 0,
$$

one shows, by applying Lemma B.1 that $\mathfrak{g}$ fits into a corresponding exact sequence

$$
0 \rightarrow 0 \rightarrow \mathfrak{g} \stackrel{b}{\rightarrow} \mathfrak{t} \rightarrow 0 .
$$

Here $b$ is the restriction of the canonical projection $J^{2}(T M) \rightarrow J^{1}(T M)$. This establishes (2).

5.9. Projective structures. Recall that two linear connections $\nabla, \nabla^{\prime}$ are projectively equivalent if their geodesics coincide as unparameterized curves. Equivalently their difference $\nabla-\nabla^{\prime}$, which may be viewed as a section of

$$
T^{*} M \otimes T^{*} M \otimes T M \subset J^{1}(T M)^{*} \otimes T^{*} M \otimes T M,
$$

should take its values in the subbundle $\Sigma_{0}:=\left(\operatorname{Alt}^{2}(T M) \otimes T M\right) \oplus j_{\mathrm{S}}\left(T^{*} M\right)$ of

$$
T^{*} M \otimes T^{*} M \otimes T M \cong\left(\operatorname{Alt}^{2}(T M) \otimes T M\right) \oplus\left(\operatorname{Sym}^{2}(T M) \otimes T M\right) .
$$

Here $j_{\mathrm{S}}: T^{*} M \rightarrow \operatorname{Sym}^{2}(T M) \otimes T M$ is the embedding defined by

$$
j_{\mathrm{S}}(\alpha)\left(V_{1}, V_{2}\right):=\alpha\left(V_{1}\right) V_{2}+\alpha\left(V_{2}\right) V_{1} .
$$

A projective structure is a projective equivalence class of linear connections; since $\Sigma_{0}$ contains $\operatorname{Alt}^{2}(T M) \otimes T M$, each such class has a torsion-free representative $\nabla$.

Let $\nabla$ be a torsion-free linear connection on $T M$ and $\langle\nabla\rangle$ the corresponding projective structure. To specify the structure it suffices to specify the affine subbundle

$$
\nabla+\Sigma_{0} \subset J^{1}(T M)^{*} \otimes T^{*} M \otimes T M,
$$

which we also denote by $\langle\nabla\rangle$. As explained in 5.8 above, we have $J^{2}(T M) \subset$ $J^{1}\left(J^{1}(T M)\right)$ acting on $J^{1}(T M)^{*} \otimes T^{*} M \otimes T M$ and can therefore define the isotropy $\mathfrak{g} \subset J^{2}(T M)$ of $\langle\nabla\rangle$; see 5.1] Arguing as in the proof of Proposition 5.8(1), one shows that the symmetries of $\mathfrak{g}$ are the prolonged infinitesimal isometries of $\langle\nabla\rangle$. 
It is not hard to see that $\mathfrak{g}$ has $j_{\mathrm{S}}\left(T^{*} M\right) \cong T^{*} M$ as a structure kernel and in fact that

$$
\mathfrak{g}=\mathfrak{g}_{\nabla} \oplus j_{\mathrm{S}}\left(T^{*} M\right)
$$

where $\mathfrak{g}_{\nabla} \subset J^{2}(T M)$ denotes the isotropy of $\nabla$ (denoted $\mathfrak{g}$ in 5.8). The Cartan connection $\nabla^{(1)}$ on $J^{1}(T M)$ in Corollary 5.8 is a generator of $\mathfrak{g}_{\nabla}$ and consequently a generator of $\mathfrak{g}$ as well. An explicit formula appears in 11.5.

5.10. $G$-structures. Let $G$ be a subgroup of $\operatorname{GL}(n, \mathbb{R})$, where $n$ is the dimension of $M$. A $G$-structure on $M$ is a $G$-reduction $P$ of the bundle of (absolute) frames on $M$; see, e.g., [11. In particular, $P$ is a principal $G$-bundle, so that $\mathfrak{g}:=T P / G$ is a transitive Lie algebroid over $M$, and the associated vector bundles of $P$ are representations of $\mathfrak{g}$; see, e.g., [12. As $P$ is a frame bundle, $T M$ will be such a representation (see below). That is, we have a Lie algebroid morphism

$$
\mathfrak{g} \rightarrow \mathfrak{g l}(T M) \stackrel{\text { ad }}{\cong} J^{1}(T M)
$$

This turns out to be injective, identifying $\mathfrak{g}$ with a subalgebroid of $J^{1}(T M)$. This infinitesimal geometric structure on $T M$ is surjective because $\mathfrak{g}$ is transitive.

The representation of $\mathfrak{g}$ on $T M$ may be described as follows. Identify sections $X$ of $\mathfrak{g}:=T P / G$ with $G$-invariant vector fields on $P$, and use the tautological 1-form on $P$ to identify sections $V$ of $T M$ with $G$-invariant $\mathbb{R}^{n}$-valued functions on $P$. Then $X \cdot V:=\mathcal{L}_{X} V$, where $\mathcal{L}$ denotes a Lie derivative on $P$.

5.11. Cartan algebroids as infinitesimal geometric structures. We have seen that all surjective infinitesimal geometric structures with a trivial structure kernel define Cartan algebroids (Theorem 2.11). Conversely, if $t$ is a Cartan algebroid with Cartan connection $\nabla$, then $\mathfrak{g}:=s_{\nabla}(\mathfrak{t}) \subset J^{1} \mathfrak{t}$ is a surjective infinitesimal geometric structure generated by $\nabla$ with a trivial structure kernel. Here $s_{\nabla}: \mathfrak{t} \rightarrow J^{1} \mathfrak{t}$ is the splitting of

$$
0 \rightarrow T^{*} M \otimes \mathfrak{t} \hookrightarrow J^{1} \mathfrak{t} \rightarrow \mathfrak{t} \rightarrow 0
$$

determined by $\nabla$.

\section{Generators, associated operators, and Bianchi identities}

Picking a generator for an infinitesimal geometric structure $\mathfrak{g} \subset J^{1} \mathfrak{t}$ allows us to identify $\mathfrak{g}$ with the direct sum $\mathfrak{t}_{1} \oplus \mathfrak{h}$ of its image $\mathfrak{t}_{1}$ and its structure kernel $\mathfrak{h}$. This greatly facilitates computations. Generators are also the appropriate connections for which to develop all the usual formalisms of differential geometry: covariant differentiation, covariant exterior differentiation, Bianchi identities, etc. (It will be natural, however, to use the more encompassing descriptor 'associated' in place of 'covariant'.) By virtue of [5.11, we obtain formalism for Cartan algebroids as a special case.

The present section, rather formal in nature, can be scanned on a first reading. In 6.1 we address the existence and uniqueness of generators and prove the theorem in 2.1, where generators were defined. In 6.2 we see how information about $\mathfrak{g}$ is encoded in $\mathfrak{t}_{1}, \mathfrak{h}$, and $\nabla$. Basic algebraic invariants of an infinitesimal geometric structure $\mathfrak{g} \subset J^{1} \mathfrak{t}$ are the vector bundles occurring as representations of $\mathfrak{g}$. Associated with these representations, and a choice of generator $\nabla$, are the associated connections and associated differential operators, described in 6.3. The latter 
generalize the divergence, gradient, etc. of Riemannian geometry when $\nabla$ is the Levi-Cevita connection. (In Section 10 we describe these objects for subriemannian contact three-manifolds.) In principle, any invariant differential operator may be expressed in terms of associated differential operators, at least in the case of surjective infinitesimal geometric structures. In 6.4 we describe the associated exterior derivative, and in 6.5 analogues of the classical Bianchi identities.

6.1. Basic properties of generators. Let $\mathfrak{g} \subset J^{1} \mathfrak{t}$ be an infinitesimal geometric structure, with structure kernel $\mathfrak{h}$, and image $\mathfrak{t}_{1} \subset \mathfrak{t}$. The projection $\mathfrak{g} \stackrel{a}{\rightarrow} \mathfrak{t}$ is of constant rank if and only if $\mathfrak{h} \subset T^{*} M \otimes \mathfrak{t}$ (or equivalently $\mathfrak{t} \subset \mathfrak{t}_{1}$ ) has constant rank (i.e., are subalgebroids).

Proposition. If $\mathfrak{g} \stackrel{a}{\rightarrow} \mathfrak{t}$ has constant rank, then:

(1) $\mathfrak{g}$ admits a generator $\nabla$.

(2) $\nabla$ is unique if and only if $\mathfrak{g}$ is surjective and $\mathfrak{h}=0$.

(3) Every $\nabla$-parallel section of $\mathfrak{t}_{1}$ is a symmetry of $\mathfrak{g}$.

Proof of the Proposition and Theorem 2.1. The constant rank hypothesis means that

$$
0 \longrightarrow \mathfrak{h} \longrightarrow \mathfrak{g} \stackrel{a}{\longrightarrow} \mathfrak{t}_{1} \longrightarrow 0
$$

is an exact sequence of vector bundles. Assuming $M$ is paracompact, it possesses a splitting $s: \mathfrak{t}_{1} \rightarrow \mathfrak{g}$ which can be extended to a splitting $s: \mathfrak{t} \rightarrow J^{1} \mathfrak{t}$ of

$$
0 \rightarrow T^{*} M \otimes \mathfrak{t} \hookrightarrow J^{1} \mathfrak{t} \rightarrow \mathfrak{t} \rightarrow 0 .
$$

To prove (1), let $\nabla$ be the corresponding linear connection on $t$.

Conclusion (2) follows readily from the correspondence between connections on $\mathfrak{t}$ and splittings of (4). To prove (3), let $s: \mathfrak{t} \rightarrow J^{1} \mathfrak{t}$ be the splitting corresponding to a generator $\nabla$, i.e., $s V=J^{1} V+\nabla V$. Then if $V \subset \mathfrak{t}_{1}$ is $\nabla$-parallel, then $J^{1} V=s V$. Since $s V$ lies in $\mathfrak{g}$, by the definition of generators, we conclude $V$ is a symmetry.

Assume $\nabla$ is a generator and $\mathfrak{h}=0$. Suppose that $V \subset \mathfrak{t}$ is a symmetry, i.e., $J^{1} V=s V-\nabla V$ is a section of $\mathfrak{g}$. Then $s V \subset \mathfrak{g}$ because $\nabla$ is a generator, implying $\nabla V \subset \mathfrak{g}$. So $\nabla V \subset\left(T^{*} M \otimes \mathfrak{t}\right) \cap \mathfrak{g}=\mathfrak{h}=0$. Symmetries are thus $\nabla$-parallel. This, together with (3), establishes Theorem 2.1.

In the remainder of this section it is tacitly assumed that all infinitesimal geometric structures have constant rank in the sense above.

6.2. Reconstructing geometric structures from generators. Knowing the structure kernel $\mathfrak{h}$, image $\mathfrak{t}_{1}$, and a generator $\nabla$ of an infinitesimal geometric structure $\mathfrak{g} \subset J^{1} \mathfrak{t}$ determines it completely: the splitting determined by the generator determines a vector bundle isomorphism $\mathfrak{g} \cong \mathfrak{t}_{1} \oplus \mathfrak{h}$ and the induced Lie algebroid structure on $\mathfrak{t}_{1} \oplus \mathfrak{h}$ can be explicitly written down; see (4) below.

It is not difficult to characterize those linear connections $\nabla$ on $\mathfrak{t}$ occurring as generators of infinitesimal geometric structures. Let $\mathfrak{t}$ be an arbitrary Lie algebroid, $\mathfrak{h}$ a subalgebroid of $T^{*} M \otimes \mathfrak{t} \subset J^{1} \mathfrak{t}$, and $\nabla$ an arbitrary linear connection on $\mathfrak{t}$. Let $\mathfrak{t}_{1} \subset \mathfrak{t}$ be an arbitrary subalgebroid. We define a $\mathfrak{t}_{1}$-connection $\bar{\nabla}$ on $T^{*} M \otimes \mathfrak{t}$ in the obvious way:

$$
\left(\bar{\nabla}_{V} \phi\right)(U)=\bar{\nabla}_{V}(\phi(U))-\phi\left(\bar{\nabla}_{V}(U)\right) ; \quad V \subset \mathfrak{t}_{1}, \phi \subset \mathfrak{h}, U \subset T M .
$$


Here $\bar{\nabla}$ on the right-hand side denotes the associated $\mathfrak{t}$-connections on $\mathfrak{t}$ and $T M$ respectively, as defined in 3.8. From the formula $s_{\nabla} V=J^{1} V+\nabla V$ and the characterization of cocurvature 4.3(2), one readily obtains:

Proposition. A linear connection $\nabla$ on a Lie algebroid $\mathfrak{t}$ is a generator of some infinitesimal geometric structure $\mathfrak{g} \subset J^{1} \mathfrak{t}$ with structure kernel $\mathfrak{h}$ and image $\mathfrak{t}_{1} \subset \mathfrak{t}$ if and only if:

(2) $\mathfrak{h} \subset T^{*} M \otimes \mathfrak{t}$ is $\bar{\nabla}$-invariant, i.e., $\bar{\nabla}_{V} \phi \subset \mathfrak{h}$ for all sections $V \subset \mathfrak{t}_{1}$ and $\phi \subset \mathfrak{h}$; and

(3) cocurv $\nabla\left(V_{1}, V_{2}\right) \subset \mathfrak{h}$ for all sections $V_{1}, V_{2} \subset \mathfrak{t}_{1}$.

If $\mathfrak{g} \subset J^{1} \mathfrak{t}$ is such an infinitesimal geometric structure, then the Lie algebroid structure of $\mathfrak{g} \cong \mathfrak{t}_{1} \oplus \mathfrak{h}$ is given by

$$
\left\{\begin{array}{c}
\#(V \oplus \phi)=\# V \\
{\left[V_{1} \oplus \phi_{1}, V_{2} \oplus \phi_{2}\right]} \\
=\left[V_{1}, V_{2}\right]_{\mathfrak{t}_{1}} \oplus\left(\left[\phi_{1}, \phi_{2}\right]_{\mathfrak{h}}+\bar{\nabla}_{V_{1}} \phi_{2}-\bar{\nabla}_{V_{2}} \phi_{1}-\operatorname{cocurv} \nabla\left(V_{1}, V_{2}\right)\right)
\end{array}\right.
$$

We recall that cocurvature was defined in 4.3 .

6.3. Associated connections and differential operators. Let $\mathfrak{g} \subset J^{1} \mathfrak{t}$ be an infinitesimal geometric structure with structure kernel $\mathfrak{h}$, image $\mathfrak{t}_{1} \subset \mathfrak{t}$, and $\nabla$ a generator of $\mathfrak{g}$. Then for each representation $E$ of $\mathfrak{g}$, i.e., for each Lie algebroid morphism $\rho: \mathfrak{g} \rightarrow \mathfrak{g l}(E)$, we have an associated $\mathfrak{t}_{1}$-connection $\bar{\nabla}$ on $E$ (a $\mathfrak{t}$-connection on $E$ if $\mathfrak{g}$ is surjective). By definition, this is the composite $\mathfrak{t}_{1} \stackrel{s_{\nabla}}{\longrightarrow} \mathfrak{g} \stackrel{\rho}{\rightarrow} \mathfrak{g l}(E)$, where $s_{\nabla}: \mathfrak{t} \rightarrow J^{1} \mathfrak{t}$ is the splitting of 6.1(4) corresponding to $\nabla$.

\section{Examples.}

(1) Taking $\mathfrak{g}:=J^{1} \mathfrak{t}$ and $\rho=\operatorname{ad}^{\mathfrak{t}}$, we obtain

$$
\begin{gathered}
\bar{\nabla}_{U} V=\operatorname{ad}_{s_{\nabla} U}^{\mathfrak{t}} V=\operatorname{ad}_{J^{1} U}^{\mathfrak{t}} V+\operatorname{ad}_{\nabla U}^{\mathfrak{t}} V, \\
\text { i.e., } \quad \bar{\nabla}_{U} V=\nabla_{\# V} U+[U, V]_{\mathfrak{t}} ; \quad U, V \subset \mathfrak{t} .
\end{gathered}
$$

This is the associated $\mathfrak{t}$-connection on $\mathfrak{t}$ already defined in 3.8 .

(2) Let $\mathfrak{g}:=J^{1} \mathfrak{t}$ act on $T M$ via the composite

$$
J^{1} \mathfrak{t} \stackrel{J^{1} \#}{\longrightarrow} J^{1}(T M) \stackrel{\operatorname{ad}^{T M}}{\longrightarrow} \mathfrak{g r}(T M) .
$$

Then we similarly compute

$$
\bar{\nabla}_{U} W=\# \nabla_{W} U+[\# U, W]_{T M} ; \quad U \subset \mathfrak{t}, W \subset T M .
$$

This is the associated $\mathfrak{t}$-connection on $T M$ defined in 3.8 .

(3) An arbitrary infinitesimal geometric structure $\mathfrak{g} \subset J^{1} \mathfrak{t}$ acts on its structure kernel $\mathfrak{h}$ via bracket: $\rho_{X} Y:=[X, Y]_{\mathfrak{g}}$. (Here we are assuming that $\mathfrak{h}$ has constant rank.) The associated $\mathfrak{t}_{1}$-connection on $\mathfrak{h}$ is simply the connection $\bar{\nabla}$ appearing in Proposition 6.2 and satisfying 6.2(1).

(4) If $\mathfrak{g} \subset J^{1} \mathfrak{t}$ is an infinitesimal geometric structure and $\nabla$ a linear connection on $\mathfrak{g}$ (rather than $\mathfrak{t}$ ), then $\nabla$ generates $J^{1} \mathfrak{g}$, which acts on $\mathfrak{t}$ via the composite

$$
J^{1} \mathfrak{g} \stackrel{J^{1} a}{\longrightarrow} J^{1} \mathfrak{t} \stackrel{\mathrm{ad}^{\mathfrak{t}}}{\longrightarrow} \mathfrak{g l}(\mathfrak{t}),
$$

where $a: \mathfrak{g} \rightarrow \mathfrak{t}$ is the projection. The associated $\mathfrak{g}$-connection $\bar{\nabla}$ on $\mathfrak{t}$ is given by $\bar{\nabla}_{X} W=a \nabla_{\# W} X+[a X, W]_{\mathfrak{t}}$. For an application, see 11.1 . 
Let a $\mathfrak{g}$-tensor be any section $\sigma \subset E$ of a $\mathfrak{g}$-representation $E$. Then, by the definition of the associated connections, we have:

Proposition. A $\mathfrak{g}$-tensor is $\mathfrak{g}$-invariant if and only if it is simultaneously $\mathfrak{h}$-invariant and $\bar{\nabla}$-parallel.

The associated derivative of a $\mathfrak{g}$-tensor $\sigma \in \Gamma(E)$ is defined to be $\bar{\nabla} \sigma \in \Gamma\left(\mathfrak{t}_{1}^{*} \otimes E\right)$, where $\bar{\nabla}$ is the associated $\mathfrak{t}_{1}$-connection on $E$. Assume $\mathfrak{g}$ is image-reduced; by this we mean that $\mathfrak{t}_{1} \subset \mathfrak{t}$ is invariant under the adjoint action of $\mathfrak{g} \subset J^{1} \mathfrak{t}$, which is true, for example, if $\mathfrak{g}$ is surjective. (Image reduction is described in 7.1.) Then $\mathfrak{t}_{1}$ is a $\mathfrak{g}$-representation, implying $\bar{\nabla} \sigma$ is another $\mathfrak{g}$-tensor. That is, the $\mathfrak{g}$-tensors will be closed under associated derivative. In particular, the derivative can be iterated to obtain higher order differential operators.

Additionally supposing that all $\mathfrak{g}$-representations may be direct-sum decomposed into $\mathfrak{g}$-representations coming from some collection $E_{i}(i \in I)$ of irreducible ones, we have

$$
\mathfrak{t}_{1}^{*} \otimes E_{i} \cong E_{n_{i 1}} \oplus E_{n_{i 2}} \oplus E_{n_{i 3}} \oplus \cdots \quad \text { (finitely many non-zero terms), }
$$

for some $n_{i j} \in I$, and obtain a corresponding decomposition,

$$
\bar{\nabla} \mid \Gamma\left(E_{i}\right)=\partial_{i 1} \oplus \partial_{i 2} \oplus \partial_{i 3} \oplus \cdots
$$

We call the differential operators $\partial_{i j}: \Gamma\left(E_{i}\right) \rightarrow \Gamma\left(E_{n_{i j}}\right)(i, j \in I)$ the associated differential operators; all differential operators which can be constructed algebraically out of associated connections $\bar{\nabla}$ are combinations of these basic ones.

If there is a canonical way in which to choose the generator $\nabla$, then the associated differential operators become invariant differential operators associated with the infinitesimal geometric structure $\mathfrak{g}$. Significant cases in point are:

(5) The case where $\mathfrak{t}$ is a Cartan algebroid discussed in 5.11. Here $\mathfrak{g}$-representations are just $\mathfrak{t}$-representations because $\mathfrak{g} \cong \mathfrak{t}$.

(6) The case where the generator $\nabla$ of $\mathfrak{g}$ is unique, i.e., Theorem 2.1 applies, reducing the situation to case (5) above.

(7) The case where the torsion tor $\bar{\nabla}$ has a natural 'normalization'; see 8.4 .

For invariant differential operators associated with subriemannian contact threemanifolds, see Section 10.

6.4. The associated exterior derivative. Let $\mathfrak{g} \subset J^{1} \mathfrak{t}$ be an infinitesimal geometric structure with structure kernel $\mathfrak{h}$. Then a differential form of type $\mathfrak{g}$ and degree $k$ is a section $\theta \subset$ Alt $^{k}\left(\mathfrak{t}_{1}\right) \otimes E$, where $\mathfrak{t}_{1} \subset \mathfrak{t}$ is the image of $\mathfrak{g}$ and $E$ some $\mathfrak{g}$-representation. (We use $\mathfrak{t}_{1}$, rather than $\mathfrak{t}$, to ensure (2) below.) The exterior derivative $d_{\bar{\nabla}} \theta \subset \operatorname{Alt}^{k}\left(\mathfrak{t}_{1}\right) \otimes E$ of $\theta$ is defined in the obvious way. For example,

$$
d_{\bar{\nabla}} \theta\left(U_{1}\right):=\bar{\nabla}_{U_{1}} \theta, \quad \text { for } k=0,
$$

and $d_{\bar{\nabla}} \theta\left(U_{1}, U_{2}\right):=\bar{\nabla}_{U_{1}}\left(\theta\left(U_{2}\right)\right)-\bar{\nabla}_{U_{2}}\left(\theta\left(U_{1}\right)\right)-\theta\left(\left[U_{1}, U_{2}\right]_{\mathfrak{t}_{1}}\right), \quad$ for $k=1$.

Wedge products of $\mathfrak{g}$-type differential forms are likewise defined by familiar formulas.

The principal invariants of the generator $\nabla$ are the $\mathfrak{g}$-type differential forms $T \subset \operatorname{Alt}^{2}\left(\mathfrak{t}_{1}\right) \otimes \mathfrak{t}$ and $\Omega \subset \operatorname{Alt}^{2}\left(\mathfrak{t}_{1}\right) \otimes \mathfrak{h}$, obtained by restricting tor $\bar{\nabla} \subset \mathrm{Alt}^{2}(\mathfrak{t}) \otimes \mathfrak{t}$ and $-\operatorname{cocurv} \nabla \subset \operatorname{Alt}^{2}(\mathfrak{t}) \otimes T^{*} M \otimes \mathfrak{t}$ to $\mathfrak{t}_{1}$ (note carefully the minus sign). 


\section{Proposition.}

(1) If $\theta_{1}$ and $\theta_{2}$ are $\mathfrak{g}$-type differential forms, then

$$
d_{\bar{\nabla}}\left(\theta_{1} \wedge \theta_{2}\right)=d_{\bar{\nabla}} \theta_{1} \wedge \theta_{2}+(-1)^{k} \theta_{1} \wedge d_{\bar{\nabla}} \theta_{2},
$$

where $k$ is the degree of $\theta_{1}$.

(2) For any $\mathfrak{g}$-type differential form $\theta$, we have

$$
d_{\bar{\nabla}}^{2} \theta=\Omega \wedge \theta
$$

Here the wedge implies a contraction $\phi \otimes \sigma \mapsto \phi \cdot \sigma: \mathfrak{h} \otimes E \rightarrow E$, defined by the representation of $\mathfrak{h}$ on $E$.

Proof of (2). The general case can easily be reduced to the $k=0$ case that we prove now. Letting $s: \mathfrak{t} \rightarrow J^{1} \mathfrak{t}$ denote the splitting of 6.1(4) determined by $\nabla$, we compute, for arbitrary $U_{1}, U_{2} \subset \mathfrak{t}_{1}$,

$$
\begin{aligned}
d_{\bar{\nabla}}^{2} \theta\left(U_{1}, U_{2}\right) & =\bar{\nabla}_{U_{1}} \bar{\nabla}_{U_{2}} \theta-\bar{\nabla}_{U_{2}} \bar{\nabla}_{U_{1}} \theta-\bar{\nabla}_{\left[U_{1}, U_{2}\right]_{\mathfrak{t}_{1}}} \theta \\
& =s U_{1} \cdot\left(s U_{2} \cdot \theta\right)-s U_{2} \cdot\left(s U_{1} \cdot \theta\right)-s\left[U_{1}, U_{2}\right]_{\mathfrak{t}_{1}} \cdot \theta \\
& =\left(s U_{1} \cdot\left(s U_{2} \cdot \theta\right)-s U_{2} \cdot\left(s U_{1} \cdot \theta\right)-\left[s U_{1}, s U_{2}\right]_{\mathfrak{g}} \cdot \theta\right) \\
& -\operatorname{cocurv} \nabla\left(U_{1}, U_{2}\right) \cdot \theta, \quad \text { by } 4.2(2) \\
& =0+\Omega\left(U_{1}, U_{2}\right) \cdot \theta .
\end{aligned}
$$

6.5. Bianchi identities. Generalizing the classical situation, the Bianchi identities below exhibit certain algebraic and differential dependencies between $T$ and $\Omega$, rooted in the equality of mixed partial derivatives. First, since $T=d_{\bar{\nabla}} i$, where $i \subset \mathfrak{t}_{1}^{*} \otimes \mathfrak{t}$ denotes the inclusion $\mathfrak{t}_{1} \subset \mathfrak{t}$, we deduce from (2) above that

$$
d_{\bar{\nabla}} T=\Omega \wedge i .
$$

Next, assume $\mathfrak{g}$ admits a representation $E$ for which the restricted representation $\mathfrak{h} \rightarrow \mathfrak{g l}(E)$ is faithful (injective), and let $\theta \subset E$ be a section, viewed as a $\mathfrak{g}$-type differential form of degree zero. Then, combining parts (11) and (2) of the preceding proposition, we obtain $d_{\bar{\nabla}}^{3} \theta=d_{\bar{\nabla}} \Omega \wedge \theta+\Omega \wedge d_{\bar{\nabla}} \theta$. Applying part (2) again, we conclude that $d_{\bar{\nabla}} \Omega \wedge \theta=0$. Since $\theta$ is arbitrary and $\mathfrak{h}$ acts faithfully on $E$, we obtain

$$
d_{\bar{\nabla}} \Omega=0 .
$$

A little manipulation allows us to write (11) and (2) in the form

$$
\begin{array}{rlr}
\left(\bar{\nabla}_{U_{3}} T\right)\left(U_{1}, U_{2}\right)+ & T\left(T\left(U_{1}, U_{2}\right), U_{3}\right) & \\
& +\Omega\left(U_{1}, U_{2}\right) \# U_{3}+1-2-3 \text {-cyclic terms }=0 & \text { (Bianchi I) }, \\
\left(\bar{\nabla}_{U_{3}} \Omega\right)\left(U_{1}, U_{2}\right)+ & \Omega\left(T\left(U_{1}, U_{2}\right), U_{3}\right) & \\
+1-2-3 \text {-cyclic terms }=0 & \text { (Bianchi II). }
\end{array}
$$

Example. If $\nabla$ is the Cartan Connection on some Lie algebroid $\mathfrak{t}$ and $\mathfrak{g} \subset J^{1} \mathfrak{t}$ the corresponding infinitesimal geometric structure (see 5.11), then $T=\operatorname{tor} \bar{\nabla}$ and $\Omega=0$. Bianchi I becomes

$$
\left(\bar{\nabla}_{U_{3}} \text { tor } \bar{\nabla}\right)\left(U_{1}, U_{2}\right)+\text { tor } \bar{\nabla}\left(\text { tor } \bar{\nabla}\left(U_{1}, U_{2}\right), U_{3}\right)+1-2-3 \text {-cyclic terms }=0 \text {. }
$$




\section{ELEMENTARY REDUCTION AND IMAGE REDUCTION}

In this section we study elementary reduction, as well as a cruder alternative we call image reduction. These techniques are useful when an infinitesimal geometric structure fails to be surjective, and in particular to intransitive infinitesimal geometric structures on $T M$. A simple application to smooth functions on a Riemannian three-manifold is included.

7.1. Image reduction. Let $\mathfrak{g} \subset J^{1} \mathfrak{t}$ be an infinitesimal geometric structure with structure kernel $\mathfrak{h} \subset T^{*} M \otimes \mathfrak{t}$ and image $\mathfrak{t}_{1} \subset \mathfrak{t}$. Assume $\mathfrak{h}$ (or equivalently $\mathfrak{t}_{1}$ ) has constant rank. Then the image reduction of $\mathfrak{g}$ is simply the isotropy $\mathfrak{g}_{\mathfrak{t}_{1}} \subset J^{1} \mathfrak{g}$ of $\mathfrak{t}_{1} \subset \mathfrak{t}$, under the adjoint representation of $\mathfrak{g} \subset J^{1} \mathfrak{t}$ on $\mathfrak{t}$. It is not hard to show that image reduction is cruder than elementary reduction, as defined in 2.4, and described further below. Nevertheless, it is usually easier to apply image reduction and this may simplify the subsequent application of elementary reduction.

7.2. Elementary reduction. With $\mathfrak{g} \subset J^{1} \mathfrak{t}, \mathfrak{h}$, and $\mathfrak{t}_{1}$ as above, let $\mathfrak{g}_{1}$ denote the elementary reduction of $\mathfrak{g}$ (see 2.4). The structure kernel of $\mathfrak{g}_{1}$ is

$$
\mathfrak{h}_{1}:=\mathfrak{h} \cap\left(T^{*} M \otimes \mathfrak{t}_{1}\right) .
$$

One can compute the image $\mathfrak{t}_{2} \subset \mathfrak{t}_{1}$ of $\mathfrak{g}_{1}$ if one knows a generator $\nabla$ of $\mathfrak{g}$ :

Proposition. There is vector bundle morphism

$$
\mathfrak{t}_{1} \stackrel{b}{\rightarrow}\left(T^{*} M \otimes \mathfrak{t}\right) /\left(T^{*} M \otimes \mathfrak{t}_{1}+\mathfrak{h}\right),
$$

whose corresponding map of section spaces is

$$
U \mapsto \nabla U \quad \bmod \left(T^{*} M \otimes \mathfrak{t}_{1}+\mathfrak{h}\right) .
$$

The morphism $b$ is independent of the choice of generator $\nabla$ and $\operatorname{ker} b=\mathfrak{t}_{2}$.

Proof. Begin by observing that the one-jet $J^{1} U(m) \in J^{1} \mathfrak{t}_{1}$ lies in $\mathfrak{g}$ if and only if $\nabla U(m)$ lies in $\mathfrak{h}$. So we define a morphism

$$
J^{1} \mathfrak{t}_{1} \stackrel{B}{\rightarrow}\left(T^{*} M \otimes \mathfrak{t}\right) / \mathfrak{h},
$$

which on sections is the map $J^{1} U \mapsto \nabla U \bmod \mathfrak{h}$, and have $\mathfrak{g}_{1}=\operatorname{ker} B$. The proposition now follows from an application of Lemma B.1 to the morphism $B$; one uses the fact that the sequence

$$
0 \rightarrow \frac{T^{*} M \otimes \mathfrak{t}_{1}}{\mathfrak{h}} \rightarrow \frac{T^{*} M \otimes \mathfrak{t}}{\mathfrak{h}} \rightarrow \frac{T^{*} M \otimes \mathfrak{t}}{T^{*} M \otimes \mathfrak{t}_{1}+\mathfrak{h}} \rightarrow 0
$$

is exact.

Remark. A significant simplification occurs if $\mathfrak{h}_{1}=\mathfrak{h}$, i.e., if $\mathfrak{h} \subset T^{*} M \otimes \mathfrak{t}$ lies entirely within $T^{*} M \otimes \mathfrak{t}_{1}$. Then we may view $b$ as the map

$$
\begin{gathered}
\mathfrak{t}_{1} \stackrel{b}{\rightarrow} T^{*} M \otimes\left(\mathfrak{t} / \mathfrak{t}_{1}\right), \\
b(U) V=\nabla_{V} U \quad \bmod \mathfrak{t}_{1} .
\end{gathered}
$$

In particular, the proposition will imply that $\mathfrak{g}_{1}=\mathfrak{g}$ if and only if $\mathfrak{t}_{1}$ is $\nabla$-invariant. In that case $\nabla$ drops to a linear connection on $\mathfrak{t}_{1}$ which generates $\mathfrak{g}$, as a surjective infinitesimal geometric structure on $\mathfrak{t}_{1}$. 
7.3. Functions on a Riemannian three-manifold. As an illustration, consider the (infinitesimal) symmetries of a smooth function $f$ on an oriented Riemannian three-manifold $M$, with metric $\sigma$. By symmetries, we mean the Killing fields of $\sigma$ preserving $f$. In the terminology of 1.3 , these are the symmetries of the joint isotropy

$$
\left(J^{1}(T M)\right)_{\sigma, f} \subset J^{1}(T M)
$$

of $\sigma$ and $f$, under the relevant representations determined by the adjoint representation of $J^{1}(T M)$ on $T M$. Any such symmetry must also preserve $d f$, and so we have an immediate reduction,

$$
\left(J^{1}(T M)\right)_{\sigma, f, d f} \subset J^{1}(T M) .
$$

Let $E:=\frac{1}{2}\|\operatorname{grad} f\|^{2}$ denote the 'energy' of $f\left(\operatorname{grad} f:=\sigma^{-1}(d f)\right)$, and assume that $d f$ and $d E$ are everywhere linearly independent. It follows that the connected components of the joint level-sets of $f$ and $E$ constitute a rank-one foliation on $M$. We denote by $T$ the unit vector field tangent to this foliation, directed so as to make $\{T, \operatorname{grad} f, \operatorname{grad} E\}$ positively oriented.

Define $J \subset T^{*} M \otimes T M$ by $J U:=\mathbf{n} \times U$, where $\mathbf{n}:=\operatorname{grad} f /\|\operatorname{grad} f\|$ (so that $J$ restricts to a complex structure on level sets of $f$ ). Then the reduction in (1) has a rank-one structure kernel spanned by $J$. Using Lemma B.1, it is not hard to see that its image is $\langle T\rangle=\operatorname{ker} d f \cap \operatorname{ker} d E$. We therefore pass to its image reduction, i.e., the joint isotropy,

$$
\mathfrak{g}:=\left(J^{1}(T M)\right)_{\sigma, f, d f,\langle T\rangle} \subset J^{1}(T M) .
$$

We observe that $\mathfrak{g}$ has a trivial structure kernel, $\mathfrak{h}=0$, and image $\mathfrak{t}_{1}:=\langle T\rangle$. To see this one applies Lemma B.1 to the morphism,

$$
\begin{gathered}
J^{1}(T M)_{\sigma, f, d f} \rightarrow T M /\langle T\rangle, \\
X \mapsto \operatorname{ad}_{X} T \bmod \langle T\rangle,
\end{gathered}
$$

which has $\mathfrak{g}$ as kernel.

As $\mathfrak{g}$ itself is evidently stable under image reduction, we now turn to elementary reduction. By Proposition 6.1, $\mathfrak{g}$ has a generator $\nabla$. Because $T$ spans the image of $\mathfrak{g}=\left(J^{1}(T M)\right)_{\sigma, f, d f,\langle T\rangle}$, we must have

$$
\bar{\nabla}_{T} \sigma=0, \quad, \bar{\nabla}_{T} \operatorname{grad} f=0 \quad \text { and } \quad \bar{\nabla}_{T} T \subset\langle T\rangle,
$$

where $\bar{\nabla}_{U} V:=\nabla_{V} U+[U, V]$. From these identities we may deduce

$$
\begin{aligned}
\nabla_{T} T & =0, \\
\nabla_{J T} T & =[J T, T], \\
\nabla_{\operatorname{grad} f} T & =[\operatorname{grad} f, T] .
\end{aligned}
$$

Assume that the image $\mathfrak{t}_{2} \subset\langle T\rangle$ of the elementary reduction $\mathfrak{g}_{1}$ of $\mathfrak{g}$ has constant rank. Then either $\mathfrak{t}_{2}=0$, in which case $\mathfrak{g}_{1}=0$ and there is no possibility for symmetry, or $\mathfrak{t}_{2}=\langle T\rangle$, in which case $\mathfrak{g}_{1}=\mathfrak{g}$. (Note here that the structure kernel of $\mathfrak{g}$ is already trivial.) According to Remark 7.2, we are in the latter case when $\langle T\rangle$ is $\nabla$-invariant. Necessary and sufficient conditions, following from (2)-(4), are

$$
[J T, T] \subset\langle T\rangle \quad \text { and } \quad[\operatorname{grad} f, T] \subset\langle T\rangle .
$$

Under these conditions, $\nabla$ descends to a Cartan connection on the line bundle $\langle T\rangle$, whose curvature is the obstruction to the existence of a Killing field preserving $f$ 
(lying necessarily along $\langle T\rangle$ ). As it turns out, this curvature generally has a single non-trivial component, $\operatorname{curv} \nabla(J T, \operatorname{grad} f)$, which the interested reader is invited to compute.

The brackets in condition (5) can be expressed in terms of the Levi-Cevita connection (e.g., $\left.[J T, T]=\nabla_{J T}^{\mathrm{L}-\mathrm{C}} T-\nabla_{T}^{\mathrm{L}-\mathrm{C}} J T\right)$, which leads to the equivalent condition that the rank-two distributions $(J T)^{\perp}$ and $T^{\perp}$ be integrable and geodesic, respectively. (A distribution is geodesic if it has a trivial second fundamental form.)

\section{Prolongation And torsion}

In this section we characterize the prolongation $\mathfrak{g}^{(1)}:=J^{1} \mathfrak{g} \cap J^{2} \mathfrak{t}$ as the joint isotropy of a tautological one-form $a$ and its 'torsion' $d a$. These tensors are analogues of classical objects bearing the same name, but the characterization is only valid when $\mathfrak{g}$ is transitive. We begin, however, with a useful characterization of the subbundle $J^{2} \mathfrak{g} \subset J^{1}\left(J^{1} \mathfrak{g}\right)$ that is completely general.

This section concludes with the reformulation of several classical constructions associated with torsion.

8.1. Prolongation. Let $\mathfrak{t}$ be an arbitrary vector bundle over $M$. Then there is a natural inclusion of vector bundles $J^{2} \mathfrak{t} \hookrightarrow J^{1}\left(J^{1} \mathfrak{t}\right)$ which takes $J^{2} W(m)$ to $J^{1}\left(J^{1} W\right)(m) ; W \subset J^{1} \mathfrak{t}$. As a basic fact one has the following:

Lemma. For any section $X \subset J^{1} \mathfrak{t}, X$ is holonomic if and only if $J^{1} X \subset J^{2} \mathfrak{t}$.

Proof. See Appendix B.4

Now the definition of prolongation, $\mathfrak{g}^{(1)}:=J^{1} \mathfrak{g} \cap J^{2} \mathfrak{t}$, of some subbundle $\mathfrak{g} \subset J^{1} \mathfrak{t}$ makes sense in general, but suppose for the moment that $t$ is a Lie algebroid and $\mathfrak{g} \subset J^{1} \mathfrak{t}$ is an infinitesimal geometric structure on $\mathfrak{t}$. Then $J^{2} \mathfrak{t} \subset J^{1}\left(J^{1} \mathfrak{t}\right)$ is a subalgebroid, implying $\mathfrak{g}^{(1)}$ is an infinitesimal geometric structure on $\mathfrak{g}$ whenever $\mathfrak{g}^{(1)}$ has constant rank. Let $W \subset \mathfrak{t}$ be a symmetry of $\mathfrak{g}$. Then a straightforward consequence of definitions is that $J^{1} W$ is a section of $\mathfrak{g}$ and, moreover, a symmetry of $\mathfrak{g}^{(1)}$. In fact, it is a consequence of the lemma above that all symmetries of $\mathfrak{g}^{(1)}$ arise in this way:

Proposition. If $\mathfrak{g} \subset J^{1} \mathfrak{t}$ is an infinitesimal geometric structure, then a section $W \subset \mathfrak{t}$ is a symmetry of $\mathfrak{g}$ if and only if $J^{1} W \subset \mathfrak{g}$ is a symmetry of $\mathfrak{g}^{(1)}$.

Since $J^{1}: \Gamma(\mathfrak{t}) \rightarrow \Gamma\left(J^{1} \mathfrak{t}\right)$ is injective, this establishes a one-to-one correspondence between the symmetries of $\mathfrak{g}$ and those of $\mathfrak{g}^{(1)}$.

8.2. More on $J^{2} \mathfrak{t}$ as a subbundle of $\left.J^{1}\left(J^{1} \mathfrak{t}\right)\right)$. The definition of $\mathfrak{g}^{(1)}$ is difficult to work with unless one has the right characterization of $J^{2} t$, as a subbundle of $J^{1}\left(J^{1} \mathfrak{t}\right)$. We now characterize $J^{2} \mathfrak{t} \subset J^{1}\left(J^{1} \mathfrak{t}\right)$ as the kernel of a natural morphism $J_{+}^{2} \mathfrak{t} \rightarrow \operatorname{Alt}^{2}(T M) \otimes \mathfrak{t}$, where $J_{+}^{2} \mathfrak{t} \subset J^{1}\left(J^{1} \mathfrak{t}\right)$ is a larger subbundle that is itself the kernel of a natural morphism $J^{1}\left(J^{1} \mathfrak{t}\right) \rightarrow T^{*} M \otimes \mathfrak{t}$. This characterization holds for an arbitrary vector bundle $\mathfrak{t}$ over $M$.

Define a differential operator

$$
\begin{aligned}
& \mathcal{D}: \Gamma\left(J^{1} \mathfrak{t}\right) \rightarrow \Gamma\left(T^{*} M \otimes \mathfrak{t}\right) \\
& \text { by } \mathcal{D} X:=X-J^{1}(a X),
\end{aligned}
$$


where $a: J^{1} \mathfrak{t} \rightarrow \mathfrak{t}$ is the natural projection. We call $\mathcal{D}$ the deviation of $X$. Notice that $X$ is holonomic if and only if $\mathcal{D} X=0$. Also, $\mathcal{D} \phi=\phi$ for all sections $\phi \subset$ $T^{*} M \otimes \mathfrak{t} \subset J^{1} \mathfrak{t}$. We write $\mathcal{D}_{V} X:=(\mathcal{D} X) V$, for $V \subset T M$ and have a Leibniz-type identity

$$
\mathcal{D}_{V}(f X)=f \mathcal{D}_{V} X+d f(V) a X,
$$

for arbitrary smooth functions $f$ on $M$.

The above construction, holding for arbitrary $\mathfrak{t}$, may be applied in the particular case that $\mathfrak{t}$ is replaced by $J^{1} \mathfrak{t}$. This delivers an operator $\Gamma\left(J^{1}\left(J^{1} \mathfrak{t}\right)\right) \rightarrow T^{*} M \otimes J^{1} \mathfrak{t}$ which will also be denoted $\mathcal{D}$. In the formulas above the projection $a$ gets replaced by the natural projection $p: J^{1}\left(J^{1} \mathfrak{t}\right) \rightarrow J^{1} \mathfrak{t}$.

Proposition (Characterization of $J^{2} \mathfrak{t} \subset J^{1}\left(J^{1} \mathfrak{t}\right)$ as a kernel). For an arbitrary vector bundle $\mathfrak{t}$, one has $J^{2} \mathfrak{t}=\operatorname{ker} \omega_{2}$, where

$$
\omega_{2}: J_{+}^{2} \mathfrak{t} \rightarrow \operatorname{Alt}^{2}(T M) \otimes \mathfrak{t}
$$

is a vector bundle morphism well defined by

$$
\left(\omega_{2} \xi\right)\left(V_{1}, V_{2}\right):=\mathcal{D}_{V_{1}} \mathcal{D}_{V_{2}} \xi-\mathcal{D}_{V_{2}} \mathcal{D}_{V_{1}} \xi-a \mathcal{D}_{\left[V_{1}, V_{2}\right]} \xi
$$

Here $\left.J_{+}^{2} \mathfrak{t} \subset J^{1}\left(J^{1} \mathfrak{t}\right)\right)$ is the kernel of the vector bundle morphism

$$
\omega_{1}: J^{1}\left(J^{1} \mathfrak{t}\right) \rightarrow T^{*} M \otimes \mathfrak{t},
$$

well defined by

$$
\left(\omega_{1} \xi\right) V:=\mathcal{D}_{V}(p \xi)-a \mathcal{D}_{V} \xi
$$

In this proposition some $\mathcal{D}$ 's are operators $\Gamma\left(J^{1} \mathfrak{t}\right) \rightarrow \Gamma\left(T^{*} M \otimes \mathfrak{t}\right)$, while others are operators $\Gamma\left(J^{1}\left(J^{1} \mathfrak{t}\right)\right) \rightarrow \Gamma\left(T^{*} M \otimes J^{1} \mathfrak{t}\right)$. All ambiguity is mitigated by the context.

Since the Proposition above is just a general fact about vector bundles, its proof is relegated to Appendix B.4.

8.3. Torsion. We now return to the case that $\mathfrak{g}$ is an infinitesimal geometric structure on a Lie algebroid $\mathfrak{t}$. Applying the general results above we obtain a characterization of $\mathfrak{g}^{(1)}$ as an isotropy subalgebroid.

Regard the restriction $a: \mathfrak{g} \rightarrow \mathfrak{t}$ of $J^{1} \mathfrak{t} \rightarrow \mathfrak{t}$ as a $\mathfrak{t}$-valued $\mathfrak{g}$-form of degree one; this is the tautological one-form. The adjoint representation of $J^{1} \mathfrak{t}$ on $\mathfrak{t}$ restricts to a representation of $\mathfrak{g}$ on $\mathfrak{t}$. So the exterior derivative $d a$ is a well defined $\mathfrak{t}$-valued $\mathfrak{g}$-form of degree two. This is the torsion of the structure. Explicitly,

$$
d a\left(X_{1}, X_{2}\right)=\operatorname{ad}_{X_{1}}^{\mathrm{t}}\left(a X_{2}\right)-\operatorname{ad}_{X_{2}}^{\mathfrak{t}}\left(a X_{1}\right)-a\left[X_{1}, X_{2}\right] .
$$

Now $a$ and $d a$ are sections of $\mathfrak{g}^{*} \otimes \mathfrak{t}$ and $\operatorname{Alt}^{2}(\mathfrak{g}) \otimes \mathfrak{t}$, respectively. We get representations of $J^{1} \mathfrak{g}$ on these spaces by taking $J^{1} \mathfrak{g}$ to act on $\mathfrak{g}$ via adjoint action, and on $\mathfrak{t}$ via the composite

$$
\begin{gathered}
J^{1} \mathfrak{g} \stackrel{p}{\rightarrow} \mathfrak{g} \hookrightarrow J^{1} \mathfrak{t} \stackrel{\mathrm{ad}^{\mathfrak{t}}}{\longrightarrow} \mathfrak{g l}(\mathfrak{t}), \\
\text { i.e., } \quad \xi \cdot W=\operatorname{ad}_{p \xi}^{\mathfrak{t}} W ; \quad X \subset \mathfrak{g}, W \subset \mathfrak{t} .
\end{gathered}
$$

Here $p: J^{1} \mathfrak{g} \rightarrow \mathfrak{g}$ denotes the canonical projection. We can accordingly define subsets of $J^{1} \mathfrak{g}$,

$$
\left(J^{1} \mathfrak{g}\right)_{a}:=\text { isotropy of } a, \quad \text { and } \quad\left(J^{1} \mathfrak{g}\right)_{d a}:=\text { isotropy of } d a .
$$

Proposition. If $\mathfrak{g}$ is transitive, then its prolongation $\mathfrak{g}^{(1)}$ is the joint isotropy of a and da, i.e., $\mathfrak{g}^{(1)}=\left(J^{1} \mathfrak{g}\right)_{a, d a}:=\left(J^{1} \mathfrak{g}\right)_{a} \cap\left(J^{1} \mathfrak{g}\right)_{d a} \subset J^{1} \mathfrak{g}$. 
Remark. If $\mathfrak{g}$ is intransitive, then $\left(J^{1} \mathfrak{g}\right)_{a, d a}$ generally has too much symmetry to be the prolongation of $\mathfrak{g}$ : every section of $\mathfrak{h} \subset T^{*} M \otimes \mathfrak{t}$ annihilating tangent vectors in the image of the anchor $\#: \mathfrak{g} \rightarrow T M$ turns out to be a symmetry of $\left(J^{1} \mathfrak{g}\right)_{a, d a}$ that is not a symmetry of $\mathfrak{g}^{(1)}$.

The Proposition is an easy corollary of Proposition 8.2 and the following observation:

Lemma. Let $\mathfrak{g} \subset J^{1} \mathfrak{t}$ be a (possible intransitive) infinitesimal geometric structure on $\mathfrak{t}$ and let $\mathcal{D}$ denote the deviation operator discussed in 8.2. Then for an arbitrary section $\xi \subset J^{1} \mathfrak{g}$, one has

(1) $(\xi \cdot a) X=\mathcal{D}_{\# X}(p \xi)-a \mathcal{D}_{\# X} \xi$, and

(2) $(\xi \cdot d a)\left(X_{1}, X_{2}\right)=d(\xi \cdot a)\left(X_{1}, X_{2}\right)+\mathcal{D}_{\# X_{1}} \mathcal{D}_{\# X_{2}} \xi-\mathcal{D}_{\# X_{2}} \mathcal{D}_{\# X_{1}} \xi-a \mathcal{D}_{\left[\# X_{1}, \# X_{2}\right]} \xi$.

Here $X, X_{1}, X_{2} \subset \mathfrak{g}$ are arbitrary sections.

Proof of the Lemma. Begin by observing that

$$
(\xi \cdot a)(X)=\operatorname{ad}_{p \xi}^{\mathfrak{t}}(a X)-a\left(\operatorname{ad}_{\xi}^{\mathfrak{g}} X\right) .
$$

Since $a: \mathfrak{g} \rightarrow \mathfrak{t}$ is a Lie algebroid morphism, the identity 3.6(3) gives us $a\left(\operatorname{ad}_{\xi}^{\mathfrak{g}} X\right)=$ $\operatorname{ad}_{\left(J^{1} a\right) \xi}^{\mathrm{t}}(a X)$, and so

$$
(\xi \cdot a)(X)=\operatorname{ad}_{p \xi-\left(J^{1} a\right) \xi}^{\mathrm{t}}(a X) .
$$

Note here that $J^{1} a: J^{1} \mathfrak{g} \rightarrow J^{1} \mathfrak{t}$ is the morphism sending $J^{1} X(m)$ to $J^{1}(a X)(m)$. Because $p \xi-\left(J^{1} a\right) \xi$ is a section of the kernel of $J^{1} \mathfrak{t} \rightarrow \mathfrak{t}$, we may view it as a section of $T^{*} M \otimes \mathfrak{t}$ and, applying 3.6(2), obtain

$$
(\xi \cdot a)(X)=\left(p \xi-\left(J^{1} a\right) \xi\right)(\# X) .
$$

On the other hand, since $\xi=J^{1}(p \xi)+\mathcal{D} \xi$, we have $\left(J^{1} a\right) \xi=J^{1}(a p \xi)+\left(J^{1} a\right)(\mathcal{D} \xi)$, implying

$$
\begin{aligned}
p \xi-\left(J^{1} a\right) \xi & =\mathcal{D}(p \xi)-\left(J^{1} a\right)(\mathcal{D} \xi) \\
\Longrightarrow\left(p \xi-\left(J^{1} a\right) \xi\right) V & =\mathcal{D}_{V}(p \xi)-a \mathcal{D}_{V} \xi, \quad V \subset T M .
\end{aligned}
$$

Combining this with (3) gives (11).

It is not too difficult to show that $\xi \cdot d a=d(\xi \cdot d a)$ whenever $\xi$ is holonomic. Therefore

$$
J^{1}(p \xi) \cdot d a=d\left(J^{1}(p \xi) \cdot a\right)=d(\xi \cdot a)-d(\mathcal{D} \xi \cdot a),
$$

because $J^{1}(p \xi)=\xi-\mathcal{D} \xi$. We now compute

$$
\begin{aligned}
\xi \cdot d a & =\left(J^{1}(p \xi)+\mathcal{D} \xi\right) \cdot d a \\
& =J^{1}(p \xi) \cdot d a+(\mathcal{D} \xi) \cdot d a \\
& =d(\xi \cdot a)-d(\mathcal{D} \xi \cdot a)+\mathcal{D} \xi \cdot d a .
\end{aligned}
$$

Note that $(\mathcal{D} \xi \cdot a) X=-a \mathcal{D}_{\# X} \xi$.

The proof of (2) now proceeds in a straightforward way, applying the kind of manipulations already encountered in the proof of (1) and is therefore omitted.

The remainder of the section describes, in Lie algebroid language, some classical constructions related to torsion. We will require the additional assumption that $\mathfrak{g} \subset J^{1} \mathfrak{t}$ is surjective. We continue to denote the structure kernel of $\mathfrak{g}$ by $\mathfrak{h}$. 
8.4. Normalizing torsion and the upper coboundary morphism. Identify $\mathfrak{g}$ with $\mathfrak{t} \oplus \mathfrak{h}$ by choosing a generator $\nabla$ of $\mathfrak{g}$. Then we obtain a corresponding identification,

$$
\operatorname{Alt}^{2}(\mathfrak{g}) \otimes \mathfrak{t} \cong\left(\operatorname{Alt}^{2}(\mathfrak{t}) \otimes \mathfrak{t}\right) \oplus\left(\mathfrak{t}^{*} \otimes \mathfrak{h}^{*} \otimes \mathfrak{t}\right) \oplus\left(\operatorname{Alt}^{2}(\mathfrak{h}) \otimes \mathfrak{t}\right),
$$

and a corresponding splitting of the torsion,

$$
d a=\operatorname{tor} \bar{\nabla} \oplus \mathrm{ev} \oplus 0 .
$$

Here $\bar{\nabla}$ denotes the associated $\mathfrak{t}$-connection on $\mathfrak{t}$ and ev is just evaluation,

$$
\mathrm{ev}(V \otimes \phi):=\phi(V) \text {. }
$$

Notice that tor $\bar{\nabla}$ is the only component of $d a$ depending on the choice of generator. Given two generators $\nabla^{1}$ and $\nabla^{2}$, their difference $\nabla^{2}-\nabla^{1}$ may be viewed as a section of $\mathfrak{t}^{*} \otimes \mathfrak{h}$ and one readily computes

$$
\operatorname{tor} \bar{\nabla}^{2}=\operatorname{tor} \bar{\nabla}^{1}+\Delta\left(\nabla^{2}-\nabla^{1}\right) \text {, }
$$

where $\Delta$ denotes the upper coboundary morphism, defined as the composite

$$
\mathfrak{t}^{*} \otimes \mathfrak{h} \hookrightarrow \mathfrak{t}^{*} \otimes T^{*} M \otimes \mathfrak{t} \stackrel{\mathrm{id} \otimes \#^{*} \otimes \mathrm{id}}{\longrightarrow} \mathfrak{t}^{*} \otimes \mathfrak{t}^{*} \otimes \mathfrak{t} \stackrel{A \otimes \mathrm{id}}{\longrightarrow} \mathrm{Alt}^{2}(\mathfrak{t}) \otimes \mathfrak{t} .
$$

Here $\#^{*}: T^{*} M \rightarrow \mathfrak{t}^{*}$ is the dual of the anchor $\#: \mathfrak{t} \rightarrow T M$ and $A(\alpha \otimes \beta):=\alpha \wedge \beta$. As an elementary consequence of (1) above, we obtain:

Proposition. If $C \subset \mathrm{Alt}^{2}(\mathfrak{t}) \otimes \mathfrak{t}$ is a complement for the image of $\Delta$, then there exists a generator $\nabla$ such that $\operatorname{tor} \bar{\nabla} \subset C$. If $\Delta$ is injective, then this generator is unique.

Note that there is no need to require that $C$ be $\mathfrak{g}$-invariant.

8.5. Intrinsic torsion and torsion reduction. Mimicking a classical construction, we define the torsion bundle,

$$
H(\mathfrak{g}):=\frac{\operatorname{Alt}^{2}(\mathfrak{t}) \otimes \mathfrak{t}}{\operatorname{im} \Delta}
$$

and call the image $\tau$ of tor $\bar{\nabla}$, under the map $\Gamma\left(\operatorname{Alt}^{2}(\mathfrak{t}) \otimes \mathfrak{t}\right) \rightarrow \Gamma(H(\mathfrak{g}))$, induced by

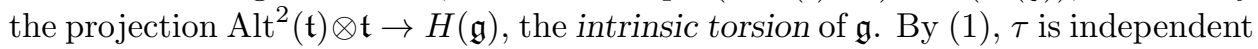
of the choice of generator, i.e., is an invariant of $\mathfrak{g}$. Since $\Delta$ is $\mathfrak{g}$-equivariant, $H(\mathfrak{g})$ is a $\mathfrak{g}$-representation whenever it is a bona fide vector bundle (has constant rank). In that case we can define the isotropy $\mathfrak{g}_{\tau} \subset \mathfrak{g}$ of $\tau$. This is the torsion reduction of $\mathfrak{g}$ and is indeed a reduction, as we show in 9.3 .

\section{9. $\Theta$-REDUCTION}

Let $\mathfrak{g} \subset J^{1} \mathfrak{t}$ be an infinitesimal geometric structure with structure kernel $\mathfrak{h}$ and let $\mathfrak{g}^{(1)} \subset J^{1} \mathfrak{g}$ be its prolongation. Recall that the $\Theta$-reduction of $\mathfrak{g}$ is simply the image of $\mathfrak{g}^{(1)}$. We will denote it by $\mathfrak{g}_{1}^{(1)}$. If $\mathfrak{g}^{(1)}$ and $\mathfrak{g}_{1}^{(1)}$ have constant rank, then $\mathfrak{g}_{1}^{(1)}$ is evidently a subalgebroid of $\mathfrak{g}$. Proposition 8.1 shows that the symmetries of $\mathfrak{g}$ are automatically symmetries of $\mathfrak{g}_{1}^{(1)} \subset \mathfrak{g}$. So $\mathfrak{g}_{1}^{(1)}$ is indeed a reduction, as claimed in Proposition 2.5 . 
For our purposes we may as well assume that $\mathfrak{g}$ is surjective; see 2.6. For simplicity, however, we strengthen this requirement:

Assumption. In this section $\mathfrak{g} \subset J^{1} \mathfrak{t}$ is a surjective infinitesimal geometric structure over a transitive Lie algebroid $\mathfrak{t}$. In particular, $\mathfrak{g}$ is transitive. Being surjective, $\mathfrak{g}$ has a structure kernel $\mathfrak{h}$ of constant rank and $\mathfrak{g}$ admits generators (Proposition $6.1(1)$.

Our chief objective is a characterization of the $\Theta$-reduction $\mathfrak{g}_{1}^{(1)}$ that does not require an explicit knowledge of $\mathfrak{g}^{(1)}$.

9.1. The lower coboundary morphism. As in torsion reduction, a 'coboundary morphism' plays a central role in $\Theta$-reduction. However, unless $\mathfrak{t}=T M$, the upper coboundary morphism $\Delta$, defined in 8.4, is not the appropriate one. Rather, we need the lower coboundary morphism $\delta$, defined as the composite

$$
T^{*} M \otimes \mathfrak{h} \hookrightarrow T^{*} M \otimes T^{*} M \otimes \mathfrak{t} \stackrel{A \otimes \mathrm{id}}{\longrightarrow} \operatorname{Alt}^{2}(T M) \otimes \mathfrak{t}
$$

where $A(\alpha \otimes \beta):=\alpha \wedge \beta$. This morphism is also a morphism of $\mathfrak{g}$-representations.

As we assume $\mathfrak{t}$ is transitive, we may, by dualizing the anchor map $\#: \mathfrak{t} \rightarrow T M$, regard $T^{*} M$ as a subbundle of $\mathfrak{t}^{*}$, and obtain natural inclusions

$$
\begin{aligned}
T^{*} M \otimes \mathfrak{h} & \hookrightarrow \mathfrak{t}^{*} \otimes \mathfrak{h}, \\
\mathrm{Alt}^{2}(T M) \otimes \mathfrak{t} & \hookrightarrow \mathrm{Alt}^{2}(\mathfrak{t}) \otimes \mathfrak{t} .
\end{aligned}
$$

With this understanding, we may regard $\delta: T^{*} M \otimes \mathfrak{h} \rightarrow \operatorname{Alt}^{2}(T M) \otimes \mathfrak{t}$ as the restriction of the upper coboundary morphism $\Delta: \mathfrak{t}^{*} \otimes \mathfrak{h} \rightarrow \operatorname{Alt}^{2}(\mathfrak{t}) \otimes \mathfrak{t}$ defined in 8.4

The analogue of the torsion bundle $H(\mathfrak{g})$ defined in 8.4 is the (variable-rank) bundle

$$
h(\mathfrak{g}):=\frac{\operatorname{Alt}^{2}(T M) \otimes \mathfrak{t}}{\operatorname{im} \delta} .
$$

Whenever $h(\mathfrak{g})$ is a genuine vector bundle (has constant rank), it is a $\mathfrak{g}$-representation. There is evidently a natural morphism $\psi: h(\mathfrak{g}) \rightarrow H(\mathfrak{g})$ making the following diagram commute:

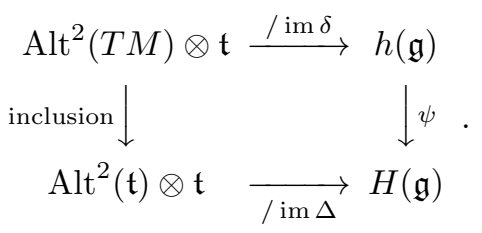

Note that $\psi$ need not be injective.

9.2. The characterization of $\mathfrak{g}_{1}^{(1)}$. The significance of the bundle $h(\mathfrak{g})$ is the existence of a natural morphism $\Theta: \mathfrak{g} \rightarrow h(\mathfrak{g})$ such that $\mathfrak{g}_{1}^{(1)}=\operatorname{ker} \Theta$. (This is the origin of our terminology ' $\Theta$-reduction'.) Recalling that $\mathfrak{g}^{(1)}:=\left(J^{1} \mathfrak{g}\right)_{a} \cap\left(J^{1} \mathfrak{g}\right)_{d a}$, our construction of $\Theta$ begins with the following observation:

(1) The isotropy $\left(J^{1} \mathfrak{g}\right)_{a} \subset J^{1} \mathfrak{g}$ is a surjective infinitesimal geometric structure with structure kernel $T^{*} M \otimes \mathfrak{h}$. 
Proof. It is not difficult to see that $\left(J^{1} \mathfrak{g}\right)_{a}$ is the kernel of

$$
\begin{aligned}
\xi & \mapsto p \xi-\left(J^{1} a\right) \xi, \\
J^{1} \mathfrak{g} & \rightarrow T^{*} M \otimes \mathfrak{t},
\end{aligned}
$$

where $p: J^{1} \mathfrak{g} \rightarrow \mathfrak{g}$ is the projection. This follows from the transitivity of $\mathfrak{g}$ and, e.g., 8.3 (3). One establishes (10) by applying Lemma B.1 to this morphism.

Now $\mathfrak{g}^{(1)}$ is the kernel of the morphism $\xi \mapsto \xi \cdot d a:\left(J^{1} \mathfrak{g}\right)_{a} \rightarrow \operatorname{Alt}^{2}(\mathfrak{g}) \otimes \mathfrak{t}$. However, it follows from 8.3(2) and transitivity that:

(2) For any $\xi \in\left(J^{1} \mathfrak{g}\right)_{a}$, the element $\xi \cdot d a \in \operatorname{Alt}^{2}(\mathfrak{g}) \otimes \mathfrak{t}$ is tensorial - i.e., drops to an element $(\xi \cdot d a)^{\vee} \in \operatorname{Alt}^{2}(T M) \otimes \mathfrak{t}$.

This means we may regard $\mathfrak{g}^{(1)}$ as the kernel of a morphism

$$
\begin{aligned}
\left(J^{1} \mathfrak{g}\right)_{a} & \stackrel{\theta}{\rightarrow} \operatorname{Alt}^{2}(T M) \otimes \mathfrak{t}, \\
\xi & \mapsto(\xi \cdot d a)^{\vee} .
\end{aligned}
$$

According to (11), the domain of $\theta$ fits into an exact sequence,

$$
0 \rightarrow T^{*} M \otimes \mathfrak{h} \hookrightarrow\left(J^{1} \mathfrak{g}\right)_{a} \rightarrow \mathfrak{g} \rightarrow 0 .
$$

Applying Lemma B.1 to the morphism $\theta$, we obtain a corresponding exact sequence,

$$
0 \rightarrow \operatorname{ker} \delta \hookrightarrow \mathfrak{g}^{(1)} \stackrel{a^{(1)}}{\longrightarrow} \operatorname{ker} \Theta \rightarrow 0
$$

where $\Theta$ is the unique morphism making the following diagram commute:

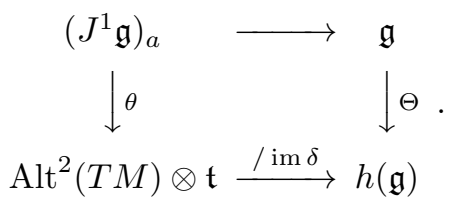

Summarizing:

Proposition. If $\mathfrak{g} \subset J^{1} \mathfrak{t}$ is surjective and $\mathfrak{t}$ is transitive, then there is a natural morphism $\Theta: \mathfrak{g} \rightarrow h(\mathfrak{g})$, constructed above, such that

$$
0 \rightarrow \operatorname{ker} \delta \hookrightarrow \mathfrak{g}^{(1)} \stackrel{a^{(1)}}{\longrightarrow} \mathfrak{g} \stackrel{\Theta}{\longrightarrow} h(\mathfrak{g})
$$

is exact. In particular, the structure kernel of $\mathfrak{g}^{(1)}$ is the kernel of the lower coboundary morphism $\delta$, while the image $\mathfrak{g}_{1}^{(1)}$ of $\mathfrak{g}^{(1)}$ (the $\Theta$-reduction of $\mathfrak{g}$ ) is the kernel of $\Theta$. If $\operatorname{ker} \delta$ and $\operatorname{ker} \Theta$ have constant rank, then $\mathfrak{g}^{(1)} \subset J^{1} \mathfrak{g}$ is an infinitesimal geometric structure.

Remark. By the proposition the structure kernel of $\mathfrak{g}^{(1)}$ lies entirely within $T^{*} M \otimes$ $\mathfrak{h} \subset T^{*} M \otimes \mathfrak{g}$ and is consequently commutative. In concrete calculations it is often useful to think of $\operatorname{ker} \delta$ as the collection of all $\sigma \in \operatorname{Sym}^{2}(T M) \otimes \mathfrak{t}$ such that $\sigma(U, \cdot) \subset T^{*} M \otimes \mathfrak{t}$ lies in $\mathfrak{h}$ for all $U \in T M$. 
9.3. The relationship with torsion. By construction $\Theta$ is an invariant of $\mathfrak{g}$. However, the construction of $\Theta$ given here is not immediately useful in computations. In Section 11 we describe $\Theta: \mathfrak{g} \rightarrow h(\mathfrak{g})$ explicitly in terms of a generator $\nabla$ of $\mathfrak{g}$. As a byproduct, we will obtain a proof of the following link between $\Theta$-reduction and intrinsic torsion:

Theorem. If $\mathfrak{g} \subset J^{1} \mathfrak{t}$ is surjective, $\mathfrak{t}$ is transitive, and $H(\mathfrak{g})$ has constant rank, then $\psi(\Theta(X))=X \cdot \tau$.

Here $\psi$ is the natural morphism $\psi: h(\mathfrak{g}) \rightarrow H(\mathfrak{g})$ defined in 9.1, and $\tau \subset H(\mathfrak{g})$ is the intrinsic torsion defined in 8.5. As a corollary we obtain the following result showing that torsion reduction is generally cruder than $\Theta$-reduction:

Corollary. Suppose that the torsion bundle $H(\mathfrak{g})$ has constant rank, so that the torsion reduction $\mathfrak{g}_{\tau}$ of $\mathfrak{g}$ is well defined. Then $\mathfrak{g}_{1}^{(1)} \subset \mathfrak{g}_{\tau}$. In particular, if the lower coboundary morphism $\delta$ has constant rank, and both $\mathfrak{g}_{\tau}$ and $\mathfrak{g}_{1}^{(1)}$ have constant rank, then $\mathfrak{g}_{\tau}$ is a reduction of $\mathfrak{g}$ in the sense of 2.3 . If $\mathfrak{t}=T M$, then $\Theta$-reduction and torsion reduction coincide.

Here the rank hypotheses and Proposition 9.2 ensure that $\mathfrak{g}^{(1)}$ has constant rank, so that Proposition 2.5 applies. However, the result presumably holds with a constant rank hypothesis on $\mathfrak{g}_{\tau}$ alone.

9.4. Structures both surjective and $\Theta$-reduced. We say that $\mathfrak{g} \subset J^{1} \mathfrak{t}$ is $\Theta$ reduced if it coincides with its $\Theta$-reduction.

Theorem. Let $\mathfrak{g} \subset J^{1} \mathfrak{t}$ be a surjective infinitesimal geometric structure on a transitive Lie algebroid $\mathfrak{t}$. Assume that $\mathfrak{g}$ is $\Theta$-reduced (equivalently, that the map $\Theta$ defined above vanishes). Assume that the associated lower coboundary morphism $\delta$ is injective. Then $\mathfrak{g}$ has an associated Cartan algebroid, namely $\mathfrak{g}$ itself, equipped with a canonical Cartan connection $\nabla^{(1)}$. The $\nabla^{(1)}$-parallel sections of $\mathfrak{g}$ coincide with the prolonged symmetries of $\mathfrak{g}$.

Proof. Proposition 2.5 implies the prolongation $\mathfrak{g}^{(1)}$ of $\mathfrak{g}$ is surjective. In addition, $\mathfrak{g}^{(1)}$ has a trivial structure kernel, because we suppose $\delta$ is injective (Proposition 9.2). Applying Theorem 2.1 to the infinitesimal geometric structure $\mathfrak{g}^{(1)}$, we obtain a Cartan connection $\nabla^{(1)}$ on $\mathfrak{g}$ whose parallel sections are the symmetries of $\mathfrak{g}^{(1)}$. These are nothing but the prolonged symmetries of $\mathfrak{g}$, by Proposition 8.1

In Proposition 11.1 we characterize $\nabla^{(1)}$ as the unique 'natural' connection on $\mathfrak{g}$ whose curvature curv $\nabla^{(1)} \subset \operatorname{Alt}^{2}(T M) \otimes \mathfrak{g}^{*} \otimes \mathfrak{g}$ takes values in $\mathfrak{h} \subset \mathfrak{g}$. A general formula expressing $\nabla^{(1)}$ in terms of a generator of $\mathfrak{g}$ will appear in 11.2 .

9.5. The special case $\mathfrak{t}=T M$. When $\mathfrak{t}=T M, \Theta$-reduction and torsion reduction are the same thing, as are the upper and lower coboundary morphisms, $\delta$ and $\Delta$. We now rewrite the above theorem accordingly, adding explicit information about the Cartan connection that we establish later in 11.4.

Here $\bar{\nabla}$ will denote the dual of $\nabla$, i.e., $\bar{\nabla}_{U} V=\nabla_{V} U+[U, V]$. We call $\mathfrak{g} \subset$ $J^{1}(T M)$ reductive if $\Delta$ has constant rank and if the image of $\Delta$ admits a $\mathfrak{g}$-invariant complement $C$. We call the generator $\nabla$ normal if tor $\bar{\nabla} \subset C$ for some such $C$. Proposition 8.4 guarantees the existence of normal generators when $\mathfrak{g}$ is reductive.

We call an arbitrary structure $\mathfrak{g} \subset \mathfrak{t}$ torsion-reduced if $\mathfrak{g}=\mathfrak{g}_{\tau}$, i.e., if the intrinsic torsion $\tau$ is $\mathfrak{g}$-invariant. 
Theorem. Let $\mathfrak{g} \subset J^{1}(T M)$ be a transitive, torsion-reduced infinitesimal geometric structure, and suppose that the associated upper coboundary morphism $\Delta$ is injective. Then $\mathfrak{g}$ has an associated Cartan algebroid, namely $\mathfrak{g}$ itself, equipped with a Cartan connection $\nabla^{(1)}$ described below. The $\nabla^{(1)}$-parallel sections of $\mathfrak{g}$ coincide with the prolonged symmetries of $\mathfrak{g}$.

Choose a generator $\nabla$ for $\mathfrak{g}$ (equivalently, choose a complement for the image of $\Delta$; see Proposition 8.4). Then:

(1) The equation

$$
\Delta(\epsilon(V \oplus \phi))=\bar{\nabla}_{V} \text { tor } \bar{\nabla}+\phi \cdot \operatorname{tor} \bar{\nabla} \quad(V \subset T M, \phi \subset \mathfrak{h} \text { arbitrary })
$$

has a unique solution morphism $\epsilon: T M \oplus \mathfrak{h} \rightarrow T^{*} M \otimes \mathfrak{h}$.

(2) Identifying $\mathfrak{g}$ with $T M \oplus \mathfrak{h}$ using the generator, we have

$$
\nabla_{U}^{(1)}(V \oplus \phi)=\left(\nabla_{U} V+\phi(U)\right) \oplus\left(\bar{\nabla}_{U} \phi+\epsilon(V \oplus \phi) U+\operatorname{curv} \bar{\nabla}(U, V)\right) .
$$

(3) If $\mathfrak{g}$ is reductive and $\nabla$ is normal, or if $\tau=0$ and $\nabla$ is torsion-free, then $\epsilon=0$ for any normal generator $\nabla$.

When one of the conditions in (3) holds, obstructions to symmetry are particularly simple to describe, as is the symmetry Lie algebra $\mathfrak{g}_{0}$ in the globally flat case. Indeed, one then computes, with the help of Proposition 6.2(4) and the Bianchi identity $d_{\bar{\nabla}} \operatorname{curv} \bar{\nabla}=0$,

$$
\begin{array}{r}
\operatorname{curv} \nabla^{(1)}\left(U_{1}, U_{2}\right)(V \oplus \phi)=0 \oplus\left(-\left(\bar{\nabla}_{V} \operatorname{curv} \bar{\nabla}+\phi \cdot \operatorname{curv} \bar{\nabla}\right)\left(U_{1}, U_{2}\right)\right), \\
\operatorname{tor} \overline{\nabla^{(1)}}\left(V_{1} \oplus \phi_{1}, V_{2} \oplus \phi_{2}\right) \\
=\left(\operatorname{tor} \bar{\nabla}\left(V_{1}, V_{2}\right)+\phi_{1}\left(V_{2}\right)-\phi_{2}\left(V_{1}\right)\right) \oplus\left(\left[\phi_{1}, \phi_{2}\right]_{\mathfrak{h}}-\operatorname{curv} \bar{\nabla}\left(V_{1}, V_{2}\right)\right) .
\end{array}
$$

Here $\overline{\nabla^{(1)}}$ denotes the representation of $\mathfrak{g}$ on itself associated with the Cartan connection $\nabla^{(1)}$ on $\mathfrak{g}$. Applying Theorem 4.6 .

Corollary. Let $\mathfrak{g} \subset J^{1}(T M)$ be an infinitesimal geometric structure satisfying the hypotheses of the above theorem, and assume either that $\mathfrak{g}$ is reductive and $\nabla$ is normal, or that $\tau=0$ and $\nabla$ is torsion-free. Let $\mathcal{U} \subset M$ be an arbitrary open set and $\mathfrak{g}_{0}$ be the Lie algebra of all symmetries of $\mathfrak{g} \mid \mathcal{U}$. Then $\operatorname{dim} \mathfrak{g}_{0} \leqslant \operatorname{rank} \mathfrak{g}$. If $U$ is simply-connected, then equality holds if and only if $\operatorname{curv} \bar{\nabla}$ is both $\mathfrak{h}$-invariant and $\bar{\nabla}$-parallel. In that case $\mathfrak{g}_{0}$ is naturally isomorphic to $T_{m} M \oplus \mathfrak{h}(m) \quad(m \in \mathcal{U}$ arbitrary) with a Lie bracket given by

$$
\begin{aligned}
{\left[V_{1} \oplus \phi_{1}, V_{2} \oplus \phi_{2}\right] } & \\
& =\left(\operatorname{tor} \bar{\nabla}\left(V_{1}, V_{2}\right)+\phi_{1}\left(V_{2}\right)-\phi_{2}\left(V_{1}\right)\right) \oplus\left(\left[\phi_{1}, \phi_{2}\right]_{\mathfrak{h}}-\operatorname{curv} \bar{\nabla}\left(V_{1}, V_{2}\right)\right) .
\end{aligned}
$$

9.6. The symmetries of Riemannian structures. Let $\mathfrak{g} \subset J^{1}(T M)$ denote the bundle of 1-symmetries of a Riemannian metric $\sigma$, as described in detail in 5.2. The upper coboundary morphism for $\mathfrak{g}$ is a map

$$
T^{*} M \otimes \mathfrak{h} \stackrel{\Delta}{\rightarrow} \operatorname{Alt}^{2}(T M) \otimes T M,
$$

where $\mathfrak{h} \subset T^{*} M \otimes T M$ is the $\mathfrak{o}(n)$-bundle of all skew-symmetric tangent space endomorphisms. This morphism is well known to be an isomorphism. For example, 
using the metric $\sigma$ to make various identifications, we may view $\Delta$ as the map

$$
\begin{aligned}
T^{*} M \otimes \operatorname{Alt}^{2}(T M) & \rightarrow \operatorname{Alt}^{2}(T M) \otimes T^{*} M \\
\alpha \otimes\left(\beta_{1} \wedge \beta_{2}\right) & \mapsto\left(\alpha \wedge \beta_{1}\right) \otimes \beta_{2}-\left(\alpha \wedge \beta_{2}\right) \otimes \beta_{1},
\end{aligned}
$$

which has explicit inverse

$$
\left(\beta_{1} \wedge \beta_{2}\right) \otimes \alpha \mapsto \frac{1}{2}\left(\beta_{1} \otimes\left(\beta_{2} \wedge \alpha\right)-\beta_{2} \otimes\left(\beta_{1} \wedge \alpha\right)-\alpha \otimes\left(\beta_{1} \wedge \beta_{2}\right)\right) .
$$

Since $\Delta$ is an isomorphism, $\mathfrak{g}$ has, by Proposition 8.4, a unique torsion-free generator $\nabla$; this is the Levi-Cevita connection. The intrinsic torsion vanishes because $H(\mathfrak{g})=0$, making $\mathfrak{g}$ torsion-reduced. Also, $\mathfrak{g}$ is trivially reductive. Applying Theorem 9.5, we obtain the Cartan connection $\nabla^{(1)}$ and related claims in 1.6] (We have $\bar{\nabla}=\nabla$ since tor $\nabla=0$.)

According to Corollary 9.5, we are in the maximally symmetric case when curv $\nabla$ is both $\mathfrak{h}$-invariant and $\nabla$-parallel. According to a well-known representationtheoretic analysis of the curvature module, this happens if and only if

$$
\operatorname{curv} \nabla\left(V_{1}, V_{2}\right)=s\left(\sigma\left(V_{1}\right) \otimes V_{2}-\sigma\left(V_{2}\right) \otimes V_{1}\right) ; \quad V_{1}, V_{2} \in T M,
$$

for some constant $s \in \mathbb{R}$ (the scalar curvature). The Lie algebra $\mathfrak{g}_{0}$ of symmetries described in the corollary is then isomorphic to the Lie algebra of infinitesimal isometries of Euclidean space, hyperbolic space, or the sphere, according to whether $s=0, s<0$, or $s>0$.

9.7. The symmetries of a conformal parallelism. Let $\omega: T M \rightarrow V$ be a global parallelism ( $V$ a vector space with the dimension of $M$ ) and let $\langle\omega\rangle \subset T^{*} M \otimes V$ be the line bundle spanned by $\omega$. A conformal parallelism is an equivalence class of absolute parallelisms, where $\omega, \omega^{\prime}: T M \rightarrow V$ are considered equivalent if $\omega^{\prime}=$ $f \omega$ for some positive function $f$. The infinitesimal isometries of the conformal parallelism having $\omega$ as a representative coincide with the symmetries of the isotropy $\mathfrak{g} \subset J^{1}(T M)$ of $\langle\omega\rangle \subset T^{*} M \otimes V$.

A straightforward application of Lemma B.1 shows that $\mathfrak{g}$ is transitive, with rank-one structure kernel $\langle\mathrm{id}\rangle \subset T^{*} M \otimes T M$.

The upper boundary morphism, given by

$$
\begin{gathered}
\Delta: T^{*} M \rightarrow \operatorname{Alt}^{2}(T M) \otimes T M, \\
\Delta(\beta)\left(U_{1}, U_{2}\right)=\beta\left(U_{1}\right) U_{2}-\beta\left(U_{2}\right) U_{1},
\end{gathered}
$$

is evidently injective $(\operatorname{dim} M \geqslant 2)$. We leave it to the reader to verify that $\mathfrak{g}$ has vanishing intrinsic torsion $\tau$ precisely when

$$
d \omega=\alpha \wedge \omega
$$

for some one-form $\alpha$. While $\alpha$ depends on the choice of representative $\omega$, the two-form $d \alpha$ does not.

Assuming $\tau=0, \mathfrak{g}$ has a unique torsion-free generator $\nabla$ (by 8.4(1) and the definition of $\tau$ ). Moreover, it is not hard to show that

$$
\bar{\nabla}_{U} \omega=\alpha(U) \omega, \quad U \subset T M,
$$

and accordingly that

$$
\operatorname{curv} \bar{\nabla}=d \alpha \otimes \mathrm{id}
$$


Applying Corollary 9.5, we are in the maximally symmetric case when $d \alpha=0$. The Lie algebra $\mathfrak{g}_{0}$ is then isomorphic to the semidirect product of the commutative Lie algebras $T_{m} M$ and $\mathbb{R}$, with $\mathbb{R}$ acting on $T_{m} M$ by scalar multiplication.

\section{Application: Subriemannian contact three-manifolds}

This section is an extended application of the general theory developed in the two preceding sections. It also includes a concrete construction of invariant differential operators, from the Lie algebroid representation viewpoint.

Equip a three-dimensional manifold $M$ with a rank-two subriemannian structure, i.e., a rank-two distribution $\mathcal{H} \subset T M$, together with a real fiber bundle inner product $\sigma \subset \operatorname{Sym}^{2}(\mathcal{H})$ on $\mathcal{H}$. We assume that $\mathcal{H}$ is a contact distribution (see below); so equipped, $M$ becomes a subriemannian contact three-manifold. This section describes the application of Cartan's method to such manifolds, à la Lie algebroids. For the $G$-structure approach, see K. Hughen's thesis 9] or [14].

Here we shall understand $\mathcal{H}$ to be transversally orientable, and understand the specification of the subriemannian structure to include a specification of transverse orientation. This amounts to the choice of a non-unique, non-vanishing one-form $\theta$ annihilating $\mathcal{H}$. The contact hypothesis means that $\theta$ is contact, i.e., $d \theta$ restricts to a symplectic structure on $\mathcal{H}$.

The infinitesimal isometries of the subriemannian contact structure are the symmetries of the infinitesimal geometric structure $J^{1}(T M)_{\mathcal{H}, \sigma}$, where $J^{1}(T M)_{\mathcal{H}} \subset$ $J^{1}(T M)$ is the isotropy of $\mathcal{H}$ and $J^{1}(T M)_{\mathcal{H}, \sigma} \subset J^{1}(T M)_{\mathcal{H}}$ is the isotropy of $\sigma$; see 5.1 .

10.1. Preliminary reduction. The symplectic structure $d \theta \mid \mathcal{H}$ orients $\mathcal{H}$. Consequently, there is a well-defined area form $d A$ determined by the subriemannian metric $\sigma$ on $\mathcal{H}$. In fact, rescaling $\theta$ by a positive function if necessary, we may arrange $d A=d \theta \mid \mathcal{H}$. A contact form $\theta$ normalized in this way is evidently an invariant of the subriemannian contact structure, implying that the isotropy

$$
\mathfrak{g}:=J^{1}(T M)_{\mathcal{H}, \sigma, d \theta} \subset J^{1}(T M)_{\mathcal{H}, \sigma}
$$

of $d \theta$ is a reduction of $J^{1}(T M)_{\mathcal{H}, \sigma}$. (This reduction is in fact the first torsionreduction of $J^{1}(T M)_{\mathcal{H}, \sigma}$.)

The subriemannian metric $\sigma$ has a canonical extension to a bona fide Riemannian metric, defined as follows: let $\mathbf{n}$ be the Reeb vector field associated with the normalized contact form $\theta$. That is,

$$
d \theta(\mathbf{n}, \cdot)=0, \quad \theta(\mathbf{n})=1 .
$$

One extends $\sigma$ so as to make $\mathbf{n}$ orthogonal to $\mathcal{H}$ and have unit length; then $\theta=$ $\sigma(\mathbf{n}):=\sigma(\mathbf{n}, \cdot)$. The easy proof of the following is left to the reader.

Proposition. The reduction $\mathfrak{g} \subset J^{1}(T M)$ above coincides with the joint isotropy of the extended metric $\sigma$ and $\mathbf{n}, \mathfrak{g}=J^{1}(T M)_{\sigma, \mathbf{n}}$.

10.2. The complex structure on $\mathcal{H}$. Let $\times$ denote the usual cross product determined by the extended metric $\sigma$ and define $J \subset T^{*} M \otimes T M$ by $J U=\mathbf{n} \times U$. Then $J$ has kernel $\langle\mathbf{n}\rangle$, image $\mathcal{H}$, and the restriction of $J$ to $\mathcal{H}$ is the complex structure on $\mathcal{H}$ relating the area form $d A$ to the subriemannian metric $\sigma$ :

$$
d A\left(U_{1}, U_{2}\right)=\sigma\left(J U_{1}, U_{2}\right) ; \quad U_{1}, U_{2} \subset \mathcal{H} .
$$


Proposition. $\mathfrak{g} \subset J^{1}(T M)$ is a surjective infinitesimal geometric structure whose structure kernel $\mathfrak{h} \subset T^{*} M \otimes T M$ is the globally trivial $\mathfrak{o}(2)$-bundle spanned by the $\mathfrak{g}$-invariant tensor $J$.

Proof. Combine the characterization of $\mathfrak{g}$ in Proposition 10.1 with the general observations of 5.3 (with $V:=\mathbf{n}$ ). In particular, the surjectivity of $\mathfrak{g}$ follows from Proposition 5.3. Alternatively, one may directly apply Lemma B.1 in the manner already demonstrated several times.

10.3. Normalizing torsion. By Proposition 10.1 a linear connection $\nabla$ on $T M$ generates $\mathfrak{g}$ if and only if $\bar{\nabla} \sigma=0$ and $\bar{\nabla} \mathbf{n}=0$; here $\sigma$ denotes the extended metric and $\bar{\nabla}_{U} V:=\nabla_{V} U+[U, V]$. Following the general discussion of 8.4 , we now fix a natural choice of generator. As a byproduct, we obtain a concrete expression for the intrinsic torsion $\tau \subset H(\mathfrak{g})$.

Let $\left(\mathcal{H}^{*} \otimes \mathcal{H}\right)_{\text {sym }} \subset \mathcal{H}^{*} \otimes \mathcal{H}$ denote the rank-three subbundle consisting of endomorphisms of fibers of $\mathcal{H}$ that are symmetric with respect to the metric $\sigma$.

\section{Proposition.}

(1) $\mathfrak{g} \subset J^{1}(T M)$ is reductive, in the sense of 9.5, and has injective upper coboundary morphism $\Delta: T^{*} M \otimes \mathfrak{h} \rightarrow \operatorname{Alt}^{2}(T M) \otimes T M$.

(2) For any generator $\nabla$ of $\mathfrak{g}$, and all vector fields $V \subset T M$, we have $\nabla_{\mathbf{n}} \mathbf{n}=0$ and $\nabla_{V} \mathbf{n} \subset \mathcal{H}$, allowing us to view $\nabla \mathbf{n}$ as a section of $\mathcal{H}^{*} \otimes \mathcal{H}$.

(3) There exists a unique and normal generator $\nabla$ such that

$$
\nabla \mathbf{n} \subset\left(\mathcal{H}^{*} \otimes \mathcal{H}\right)_{\mathrm{sym}} \quad \text { and } \quad \nabla \sigma \mid \mathcal{H}=0 .
$$

Here $\nabla \sigma \mid \mathcal{H} \subset \mathcal{H}^{*} \otimes \operatorname{Sym}^{2}(\mathcal{H})$ denotes the restriction of $\nabla \sigma \subset T^{*} M \otimes \operatorname{Sym}^{2}(T M)$.

With $\nabla$ so fixed, we have:

(4) The torsion tor $\bar{\nabla}=-$ tor $\nabla$ is given by the formula

$$
\text { tor } \bar{\nabla}\left(U_{1}+a_{1} \mathbf{n}, U_{2}+a_{2} \mathbf{n}\right)=\left(a_{1} \nabla_{U_{2}} \mathbf{n}-a_{2} \nabla_{U_{1}} \mathbf{n}\right)+d A\left(U_{1}, U_{2}\right) \mathbf{n} .
$$

Here $U_{1}, U_{2} \in \mathcal{H}, a_{1}, a_{2} \in \mathbb{R}$.

(5) There exists a natural isomorphism of $\mathfrak{g}$-representations,

$$
H(\mathfrak{g}) \cong \operatorname{Alt}^{2}(T M) \oplus\left(\mathcal{H}^{*} \otimes \mathcal{H}\right)_{\text {sym }},
$$

with respect to which the intrinsic torsion of $\mathfrak{g}$ takes the form

$$
\tau=d \theta \oplus \nabla \mathbf{n} .
$$

(6) The intrinsic torsion component $\nabla \mathbf{n}$ can be interpreted as a transverse derivative of the subriemannian metric $\sigma$ :

$$
\sigma\left(\nabla_{U_{1}} \mathbf{n}, U_{2}\right)=\left(\nabla_{\mathbf{n}} \sigma\right)\left(U_{1}, U_{2}\right) ; \quad U_{1}, U_{2} \subset \mathcal{H} .
$$

The proposition is established by analyzing the coboundary morphism $\Delta$ in detail, identifying a natural $\mathfrak{g}$-invariant complement for its image, and applying Proposition 8.4. This analysis is not hard but is a little tedious, and it is relegated to Appendix B.3. For the interested reader, we include there a formula for the normalized generator $\nabla$ in terms of the Levi-Cevita connection associated with the extended metric $\sigma$. 
10.4. Bianchi identities and low weight differential operators. We shall write down Bianchi identities for the normalized generator $\nabla$ using certain natural invariant differential operators, the systematic construction of which is deferred to 10.6. For now, we merely list those operators of 'weight' two or less.

Let $\mathcal{F}(M)=\Gamma(\mathbb{R} \times M)$ denote the space of all smooth functions. Then we define $\partial_{\mathbf{n}}: \mathcal{F}(M) \rightarrow \mathcal{F}(M), \operatorname{grad}_{\mathcal{H}}: \mathcal{F}(M) \rightarrow \Gamma(\mathcal{H})$, and $\operatorname{div}_{\mathcal{H}}: \Gamma(\mathcal{H}) \rightarrow \mathcal{F}(M)$ in a familiar way:

$$
\begin{gathered}
\partial_{\mathbf{n}} f:=d f(\mathbf{n}), \\
\operatorname{grad}_{\mathcal{H}} f:=\sigma^{-1}(d f \mid \mathcal{H}), \quad \operatorname{div}_{\mathcal{H}} U:=\operatorname{trace}(\bar{\nabla} U \mid \mathcal{H}) .
\end{gathered}
$$

Additionally, it is convenient to write $\operatorname{curl}_{\mathcal{H}} U:=\operatorname{div}_{\mathcal{H}}(J U)$.

Next, let $\mathcal{H}_{2} \subset\left(\mathcal{H}^{*} \otimes \mathcal{H}\right)_{\text {sym }}$ denote the rank-two subbundle of trace-free elements. Equivalently, $\mathcal{H}_{2}$ consists of those elements of $\mathcal{H}^{*} \otimes \mathcal{H}$ that are $\mathbb{C}$-antilinear: $\mathcal{H}_{2}=\overline{\mathcal{H}}^{* \mathbb{C}} \otimes_{\mathbb{C}} \mathcal{H}$. Like $\mathcal{H}, \mathcal{H}_{2}$ is a $\mathfrak{g}$-representation and a complex line bundle. However, while $\operatorname{ad}_{J} U=i U$, for $U \subset \mathcal{H}$ (by definition), we have, for $q \subset \mathcal{H}_{2}$, $\left(\operatorname{ad}_{J} q\right) U=J(q U)-q(J U)=2 J(q U)$, i.e., $\operatorname{ad}_{J} q=2 i q$.

We are now ready to define two invariant operators $\partial_{+}: \Gamma(\mathcal{H}) \rightarrow \Gamma\left(\mathcal{H}_{2}\right)$ and $\partial_{-}: \Gamma\left(\mathcal{H}_{2}\right) \rightarrow \Gamma(\mathcal{H})$ according to

$$
\begin{gathered}
\left(\partial_{+} U\right) V=\frac{1}{2}\left(\bar{\nabla}_{V} U+J \bar{\nabla}_{J V} U\right), \\
\left(\bar{\nabla}_{U_{1}} q\right) U_{2}-\left(\bar{\nabla}_{U_{2}} q\right) U_{1}=d A\left(U_{1}, U_{2}\right) \partial_{-} q .
\end{gathered}
$$

Associated with the normalized generator $\nabla$ of $\mathfrak{g}$ are its two fundamental invariants $T:=\operatorname{tor} \bar{\nabla}$ and $\Omega:=\operatorname{cocurv} \nabla=-\operatorname{curv} \bar{\nabla}$, which satisfy the Bianchi identities of 6.5(3) and 6.5(4). Of course these are also invariants of the subriemannian contact structure. According to Proposition 10.3(4), $T$ depends only on the previously identified invariant $\nabla \mathbf{n}$. As it turns out, one component of $\Omega$ is a new invariant function. Recalling that $\Omega\left(U_{1}, U_{2}\right) \subset \mathfrak{h}$ for all $U_{1}, U_{2} \subset T M$ (Proposition 6.2(3)) and that $d A$ spans $\operatorname{Alt}^{2}(\mathcal{H})$, there is a real-valued function $\kappa$ well defined by

$$
\Omega\left(U_{1}, U_{2}\right) U_{3}=-\kappa d A\left(U_{1}, U_{2}\right) J U_{3} ; \quad U_{1}, U_{2}, U_{3} \subset \mathcal{H} .
$$

Proposition (Bianchi identities).

(2) $\operatorname{trace}(\nabla \mathbf{n})=0$, i.e., $\nabla \mathbf{n} \subset \mathcal{H}_{2}$.

(3) $\partial_{\mathbf{n}} \kappa=-\frac{1}{2} \operatorname{curl}_{\mathcal{H}}\left(\partial_{-}(\nabla \mathbf{n})\right)$.

(4) The cocurvature of $\nabla$ is given by

$$
\begin{aligned}
& \Omega\left(U_{1}+a_{1} \mathbf{n}, U_{2}+a_{2} \mathbf{n}\right)\left(U_{3}+a_{3} \mathbf{n}\right) \\
&=\left(-\kappa d A\left(U_{1}, U_{2}\right)+\frac{1}{2} \sigma\left(\partial_{-}(\nabla \mathbf{n}), a_{1} U_{2}-a_{2} U_{1}\right)\right) J U_{3} ; \\
& U_{1}, U_{2}, U_{3} \in \mathcal{H}, a_{1}, a_{2}, a_{3} \in \mathbb{R} .
\end{aligned}
$$

Proof. Proposition 10.3(4) states that

$$
T\left(U_{1}+a_{1} \mathbf{n}, U_{2}+a_{2} \mathbf{n}\right)=\left(a_{1} \nabla_{U_{2}} \mathbf{n}-a_{2} \nabla_{U_{1}} \mathbf{n}\right)+d A\left(U_{1}, U_{2}\right) \mathbf{n} .
$$

A little multilinear algebra determines that $\Omega$ has the general form

$$
\Omega\left(U_{1}+a_{1} \mathbf{n}, U_{2}+a_{2} \mathbf{n}\right)\left(U_{3}+a_{3} \mathbf{n}\right)=-\left(\kappa d A\left(U_{1}, U_{2}\right)+\omega\left(a_{1} U_{2}-a_{2} U_{1}\right)\right) J U_{3},
$$

for some section $\omega \subset \mathcal{H}^{*}$ and some $\kappa$ as above. The Bianchi identities of 6.5)(3) and 6.5(4) are equations in bundle-valued three-forms. An arbitrary three-form $\lambda$ 
on $M$ vanishes if and only if $\lambda\left(U_{1}, U_{2}, \mathbf{n}\right)=0$ for all sections $U_{1}, U_{2} \subset \mathcal{H}$. Applying this fact to the Bianchi identities gives

$$
\begin{aligned}
& \left.\left.\begin{array}{l}
d A\left(\nabla_{U_{1}} \mathbf{n}, U_{2}\right)+d A\left(U_{1}, \nabla_{U_{2}} \mathbf{n}\right)=0 \\
\left(\bar{\nabla}_{U_{1}}(\nabla \mathbf{n})\right) U_{2}-\left(\bar{\nabla}_{U_{2}}(\nabla \mathbf{n})\right) U_{1}=2 J\left(\omega\left(U_{1}\right) U_{2}-\omega\left(U_{2}\right) U_{1}\right)
\end{array}\right\} \quad \text { (Bianchi I }\right), \\
& \left(\partial_{\mathbf{n}} \kappa\right) d A\left(U_{1}, U_{2}\right)=\left(\bar{\nabla}_{U_{1}} \omega\right) U_{2}-\left(\bar{\nabla}_{U_{2}} \omega\right) U_{1} \quad \text { (Bianchi II), }
\end{aligned}
$$

for arbitrary $U_{1}, U_{2} \subset \mathcal{H}$. From the first of these equations one deduces (2); from the second equation, that $\omega=-\frac{1}{2} \sigma\left(\partial_{-}(\nabla \mathbf{n})\right)$; and from the third, that $\partial_{\mathbf{n}} \kappa=$ $\operatorname{curl}_{\mathcal{H}}\left(\sigma^{-1}(\omega)\right)$.

10.5. The maximally symmetric case. Since $d \theta$ is already $\mathfrak{g}$-invariant, Proposition 10.3(5) implies that $\mathfrak{g}$ is torsion-reduced if and only if $\nabla \mathbf{n}$ is $\mathfrak{g}$-invariant. But $\mathfrak{g}$-invariance implies $\mathfrak{h}$-invariance, which, by Proposition 10.2, is the same thing as $\mathbb{C}$-linearity. However, $\nabla \mathbf{n}$ is $\mathbb{C}$-antilinear, by (2) above, and we conclude that $\mathfrak{g}$ is torsion-reduced if and only if $\nabla \mathbf{n}=0$.

Suppose that $\nabla \mathbf{n}=0$. Then $\mathbf{n}$ is automatically a symmetry of $\mathfrak{g}$, and hence an infinitesimal isometry of the subriemannian contact structure. This is a consequence of Proposition 6.1(3). Note that if the rank-one foliation generated by $\mathbf{n}$ fibrates over some surface $\Sigma$, then the invariant function $\kappa$ drops to a function on $\Sigma$, by (3) above. In any case, Theorem 9.5 applies, because of Proposition 10.3(1). Using the formula for $\Omega=-\operatorname{curv} \bar{\nabla}$ above, one applies this theorem and its corollary to obtain:

Proposition (Compare with 9]). Suppose $\nabla \mathbf{n}=0$. Then $\mathfrak{g} \subset J^{1}(T M)$ has an associated Cartan algebroid, namely $\mathfrak{g}$ itself. If $\mathcal{U} \subset M$ is an arbitrary open subset, and $\mathfrak{g}_{0}$ the Lie algebra of all infinitesimal isometries of the subriemannian contact structure on $\mathcal{U}$, then $\operatorname{dim} \mathfrak{g}_{0} \leq \operatorname{rank} \mathfrak{g}=4$. If $\mathcal{U}$ is simply-connected, then equality holds if and only if the function $\kappa$ defined by (1) above is constant. In that case $\mathfrak{g}_{0} \cong \mathfrak{b} \times \mathbb{R}$ (direct product), where $\mathfrak{b}$ is the Lie algebra of infinitesimal isometries (Killing fields) of the Euclidean plane, hyperbolic plane, or sphere, according to whether $\kappa=0, \kappa<0$, or $\kappa>0$.

10.6. Invariant differential operators. The normalized generator $\nabla$ determines associated invariant differential operators, as explained in 6.3. These operators will be invariants of the subriemannian contact structure analogous to the divergence, gradient, curl, etc., of a Riemannian three-manifold.

Noting that the structure kernel $\mathfrak{h} \subset \mathfrak{g}$ of $\mathfrak{g}$ is an $\mathfrak{o}(2)$-bundle, we construct irreducible representations of $\mathfrak{g}$ by mimicking a known construction of the irreducible representations of the Lie algebra $\mathfrak{o}(2)$. At least locally, this construction accounts for all irreducible representations of $\mathfrak{g}$.

Define $\mathcal{H}_{0}:=\mathbb{C} \times M, \mathcal{H}_{1}:=\mathcal{H}$, and define $\mathcal{H}_{2}$ as in 10.4 above. More generally, we define

$$
\mathcal{H}_{k}:=\operatorname{Sym}_{\mathbb{C}}^{k-1}(\overline{\mathcal{H}}) \otimes_{\mathbb{C}} \mathcal{H} ; \quad k \geqslant 1,
$$

where $\overline{\mathcal{H}}$ is $\mathcal{H}$ with the complex structure $-J$. Each $\mathcal{H}_{k}$ is simultaneously a $\mathfrak{g}$ representation and a complex line bundle, the two structures being related according to

$$
\operatorname{ad}_{J} q=k i q ; \quad q \subset \mathcal{H}_{k}, k \geqslant 0 .
$$

Every $\mathcal{H}_{k}$ is irreducible as a (real) $\mathfrak{g}$-representation, except $\mathcal{H}_{0}$, which is two copies of the irreducible trivial representation $\mathbb{R} \times M$. 
Recall that for each section $q \subset E$ of an irreducible $\mathfrak{g}$-representation $E$, our objective is to derive the decomposition of $\bar{\nabla} q \subset T^{*} M \otimes E$ corresponding to the decomposition of $T^{*} M \otimes E$ into irreducibles. When $E=\mathbb{R} \times M, q$ is just a real function and, because $T M=\mathcal{H} \otimes\langle\mathbf{n}\rangle$, the decompositions are straightforward:

$$
T^{*} M \otimes(\mathbb{R} \times M) \cong \mathcal{H} \oplus(\mathbb{R} \times M), \quad \bar{\nabla} q=\operatorname{grad}_{\mathcal{H}} q \oplus \partial_{\mathbf{n}} q,
$$

where $\operatorname{grad}_{\mathcal{H}} q$ and $\partial_{\mathbf{n}} q$ are as defined previously. To analyze the case $E=\mathcal{H}_{k}$ $(k \geqslant 1)$ we need the decomposition of $\mathcal{H}^{*} \otimes \mathcal{H}_{k}$ into irreducibles. To this end, note that for any $k \geqslant 1$, we have an exact sequence,

$$
0 \rightarrow \mathcal{H}_{k+1} \hookrightarrow \mathcal{H}^{*} \otimes \mathcal{H}_{k} \stackrel{\pi_{-}}{\longrightarrow} \mathcal{H}_{k-1} \rightarrow 0,
$$

where $\pi_{-}$is defined implicitly via

$Q\left(U_{1}, U_{2}, V_{1}, \ldots, V_{k-2}\right)-Q\left(U_{2}, U_{1}, V_{1}, \ldots, V_{k-2}\right)=d A\left(U_{1}, U_{2}\right)\left(\pi_{-} Q\right)\left(V_{1}, \ldots, V_{k-2}\right)$,

for $k \geqslant 2$, and via

$$
\left\langle Q\left(U_{1}\right), U_{2}\right\rangle-\left\langle Q\left(U_{2}\right), U_{1}\right\rangle=d A\left(U_{1}, U_{2}\right)\left(\pi_{-} Q\right)
$$

for $k=1$. In the latter case we are using the Hermitian product on $\mathcal{H}$ defined by $\langle U, V\rangle=\sigma(U, V)-i d A(U, V)$.

A compatible pair of splitting morphisms $\mathcal{H}_{k+1} \stackrel{\pi_{+}}{\longleftarrow} \mathcal{H}^{*} \otimes \mathcal{H}_{k} \stackrel{s}{\leftarrow} \mathcal{H}_{k-1}$ are defined as follows:

$$
\left(\pi_{+} Q\right)\left(U, V_{1}, \ldots, V_{k-1}\right)=\frac{1}{2}\left(Q\left(U, V_{1}, \ldots, V_{k-1}\right)+i Q\left(J U, V_{1}, \ldots, V_{k-1}\right)\right),
$$

for all $k \geqslant 1$, and

$$
(s q)\left(U_{1}, U_{2}, V_{1}, \ldots, V_{k-2}\right)=\frac{i}{2}\left\langle U_{1}, U_{2}\right\rangle q\left(V_{1}, \ldots, V_{k-2}\right),
$$

for $k \geqslant 2$, while

$$
(s q) U=\frac{1}{2} q U,
$$

for $k=1$ ( $q$ a $\mathbb{C}$-valued function).

Let $q$ be a section of $\mathcal{H}_{k}$. Then we have a restriction $\bar{\nabla} q \mid \mathcal{H} \subset \mathcal{H}^{*} \otimes \mathcal{H}_{k}$. As (11) splits, we have $\mathcal{H}^{*} \otimes \mathcal{H}_{k} \cong \mathcal{H}_{k-1} \oplus \mathcal{H}_{k+1}$ and are led to define $\partial_{+} q:=\pi_{+}(\bar{\nabla} q \mid \mathcal{H})$ and $\partial_{-} q:=\pi_{-}(\bar{\nabla} q \mid \mathcal{H})$. That is, $\partial_{+} q \subset \mathcal{H}_{k+1}$ and $\partial_{-} q \subset \mathcal{H}_{k-1}$ are defined by

$$
\left(\partial_{+} q\right)\left(U, V_{1}, \ldots, V_{k-1}\right)=\frac{1}{2}\left(\left(\bar{\nabla}_{U} q\right)\left(V_{1}, \ldots, V_{k-1}\right)+i\left(\bar{\nabla}_{J U} q\right)\left(V_{1}, \ldots, V_{k-1}\right)\right),
$$

for any $k \geqslant 1$,

$$
\begin{aligned}
\left(\bar{\nabla}_{U_{1}} q\right)\left(U_{2}, V_{1}, \ldots, V_{k-2}\right)-\left(\bar{\nabla}_{U_{2}} q\right)\left(U_{1}, V_{1}, \ldots, V_{k-2}\right) & \\
& =d A\left(U_{1}, U_{2}\right)\left(\partial_{-} q\right)\left(V_{1}, \ldots, V_{k-2}\right)
\end{aligned}
$$

for $k \geqslant 2$, and

$$
\left\langle\bar{\nabla}_{U_{1}} q, U_{2}\right\rangle-\left\langle\bar{\nabla}_{U_{2}} q, U_{1}\right\rangle=d A\left(U_{1}, U_{2}\right) \partial_{-} q
$$

for $k=1$. This last formula simply means, for $q \subset \mathcal{H}$, that

$$
\partial_{-} q=\operatorname{curl}_{\mathcal{H}}(q)-i \operatorname{div}_{\mathcal{H}}(q) .
$$

Finally, for any section $q \subset \mathcal{H}_{k}$ and any $k \geqslant 1$, we define $\partial_{\mathbf{n}} q:=\bar{\nabla}_{\mathbf{n}} q$, another section of $\mathcal{H}_{k}$. 
Combining our observation $\mathcal{H}^{*} \otimes \mathcal{H}_{k} \cong \mathcal{H}_{k-1} \oplus \mathcal{H}_{k+1}$ with the decomposition $T M=\mathcal{H} \otimes\langle\mathbf{n}\rangle$, we now obtain:

Proposition. For any $k \geqslant 1$, we have a natural decomposition

$$
T^{*} M \otimes \mathcal{H}_{k} \cong \mathcal{H}_{k-1} \oplus \mathcal{H}_{k} \oplus \mathcal{H}_{k+1}
$$

and a corresponding decomposition of operators

$$
\bar{\nabla}=\partial_{-} \oplus \partial_{\mathbf{n}} \oplus \partial_{+},
$$

where $\partial_{-}, \partial_{\mathbf{n}}$ and $\partial_{+}$are defined above.

\section{Advanced PRolongation THEORY}

This section describes in detail the prolongation $\mathfrak{g}^{(1)}$ of a surjective infinitesimal geometric structure $\mathfrak{g} \subset J^{1} \mathfrak{t}$. This description, obtained by 'prolonging' a generator of $\mathfrak{g}$ to a generator of $\mathfrak{g}^{(1)}$, is concrete enough to permit computations in examples. The central result, Theorem 11.2 is probably the most widely applicable in such calculations. This theorem is used to prove several theoretical results stated earlier; some special cases are also considered.

We assume throughout that $\mathfrak{t}$ is a transitive Lie algebroid. For basic implications, see the Assumption in Section 9. We continue to denote the structure kernel of $\mathfrak{g}$ by $\mathfrak{h}$, and the associated lower coboundary morphism, defined in 9.1 by $\delta$.

11.1. Natural connections. Call a linear connection $D$ on $\mathfrak{g} \subset J^{1} \mathfrak{t}$ natural if the associated $\mathfrak{g}$-connection on $\mathfrak{t}$ - see Example 6.3(4) - is the adjoint representation of $\mathfrak{g} \subset J^{1} \mathfrak{t}$ on $\mathfrak{t}$; in symbols, if

$$
a D_{\# V} X+[a X, V]_{\mathfrak{t}}=\operatorname{ad}_{X}^{\mathfrak{t}} V ; \quad V \subset \mathfrak{t}, X \subset \mathfrak{g} .
$$

Here $a: \mathfrak{g} \rightarrow \mathfrak{t}$ is the restricted projection $J^{1} \mathfrak{t} \rightarrow \mathfrak{t}$. The following proposition is not immediately useful in computations but is a natural intermediate result. It stands between the rather abstract Proposition 9.2 and the computationally useful Theorem 11.2 given later.

Proposition. Let $D$ be any natural connection on $\mathfrak{g}$ and consider the morphism

$$
\begin{gathered}
\mathfrak{g} \stackrel{\dot{\Theta}}{\rightarrow} \operatorname{Alt}^{2}(T M) \otimes \mathfrak{t}, \\
\dot{\Theta}(X)\left(U_{1}, U_{2}\right):=a\left(\operatorname{curv} D\left(U_{1}, U_{2}\right) X\right),
\end{gathered}
$$

where $a$ is the projection $\mathfrak{g} \rightarrow \mathfrak{t}$. Then:

(1) The morphism $\Theta: \mathfrak{g} \rightarrow h(\mathfrak{g})$, defined in 9.2 , coincides with the composite

$$
\mathfrak{g} \stackrel{\dot{\Theta}}{\rightarrow} \operatorname{Alt}^{2}(T M) \otimes \mathfrak{t} \stackrel{/ \mathrm{im} \delta}{\longrightarrow} h(\mathfrak{g}) .
$$

Moreover, if $\operatorname{ker} \delta$ and $\operatorname{ker} \Theta$ have constant rank (so that $\mathfrak{g}^{(1)} \subset J^{1} \mathfrak{g}$ is an infinitesimal geometric structure, by Proposition 9.2) then:

(2) If $\Theta=0$, then all generators of $\mathfrak{g}^{(1)}$ are natural.

(3) A natural connection $D$ on $\mathfrak{g}$ generates $\mathfrak{g}^{(1)}$ if and only if

$$
\operatorname{curv} D\left(U_{1}, U_{2}\right) X \in \mathfrak{h} \quad \text { for all } X \in \operatorname{ker} \Theta \text { and } U_{1}, U_{2} \in T M \text {. }
$$

Corollary. If $\mathfrak{g} \subset J^{1} \mathfrak{t}$ is a $\Theta$-reduced infinitesimal geometric structure, then a linear connection $D$ on $\mathfrak{g}$ generates $\mathfrak{g}^{(1)}$ if and only if $D$ is natural and $\operatorname{curv} D \subset$ $\operatorname{Alt}^{2}(T M) \otimes \mathfrak{g}^{*} \otimes \mathfrak{h}$. 
Corollary. The Cartan connection $\nabla^{(1)}$ in Theorem 9.4 is the unique natural connection on $\mathfrak{g}$ such that $\operatorname{curv} \nabla^{(1)} \subset \operatorname{Alt}^{2}(T M) \otimes \mathfrak{g}^{*} \otimes \mathfrak{h}$.

The second Corollary holds because $D:=\nabla^{(1)}$ is the unique generator of $\mathfrak{g}^{(1)}$ under the hypotheses of Theorem 9.4. The Proposition's proof rests on parts (4) and (5) of the following technical result (짐 is needed in the next section).

\section{Lemma.}

(4) A linear connection $D$ on $\mathfrak{g}$ is natural if and only if it is a generator of the isotropy $\left(J^{1} \mathfrak{g}\right)_{a} \subset J^{1} \mathfrak{g}$ of $a$.

(5) If $D$ is a natural connection on $\mathfrak{g}$, then

$$
(s X \cdot d a)^{\vee}\left(U_{1}, U_{2}\right)=a\left(\operatorname{curv} D\left(U_{1}, U_{2}\right) X\right) ; \quad X \subset \mathfrak{g} .
$$

Here $s: \mathfrak{g} \rightarrow J^{1} \mathfrak{g}$ denotes the splitting of the exact sequence,

$$
0 \rightarrow T^{*} M \otimes \mathfrak{g} \rightarrow J^{1} \mathfrak{g} \rightarrow \mathfrak{g} \rightarrow 0,
$$

corresponding to $D$, and $(s X \cdot d a)^{\vee}$ is the 'reduction' of $s X \cdot d a$ as per 9.2(2).

(6) If $\nabla$ is a connection on $\mathfrak{t}$ generating $\mathfrak{g}$ and $\nabla^{\mathfrak{h}}$ is any linear connection on $\mathfrak{h}$, then, identifying $\mathfrak{g}$ with $\mathfrak{t} \oplus \mathfrak{h}$ using the generator $\nabla$, every natural connection on $\mathfrak{g}$ is of the form

$$
D_{U}(V \oplus \phi)=\left(\nabla_{U} V+\phi(U)\right) \oplus\left(\nabla_{U}^{\mathfrak{h}} \phi+\epsilon(V \oplus \phi) U\right),
$$

for some vector bundle morphism $\epsilon: \mathfrak{t} \oplus \mathfrak{h} \rightarrow T^{*} M \otimes \mathfrak{h}$.

Proof. Let $\mathcal{D}$ be the deviation operator described in 8.2 . Then

$$
[a X, V]_{\mathfrak{t}}=\operatorname{ad}_{J^{1}(a X)}^{\mathfrak{t}} V=\operatorname{ad}_{X}^{\mathfrak{t}} V-\mathcal{D}_{\# V} X ; \quad X \subset \mathfrak{g}, V \subset \mathfrak{t} .
$$

Taking care not to confuse $D$ 's with $\mathcal{D}$ 's, we also have

$$
a D_{\# V} X=a\left(s X-J^{1} X\right)(\# V)=a(\mathcal{D}(s X)) \# V=a \mathcal{D}_{\# V}(s X) .
$$

Here $s$ is the splitting in (5). Combining this with (7) gives

$$
a D_{\# V} X+[a X, V]_{\mathfrak{t}}-\operatorname{ad}_{X}^{\mathfrak{t}} V=a \mathcal{D}_{\# V}(s X)-\mathcal{D}_{\# V}(p(s X)) .
$$

The claim in (4) now follows from 8.3(1) and transitivity. Similarly, (5) may be derived as a consequence of 8.3(2) and transitivity. One checks that the connection in (6) is natural and, with the help of (4) and 9.2(1), that all possibilities are covered, establishing (6).

Proof of the Proposition. By (4), $D$ generates $\left(J^{1} \mathfrak{g}\right)_{a}$. Recalling that $\left(J^{1} \mathfrak{g}\right)_{a}$ is surjective (see 9.2(1) ), let $s: \mathfrak{g} \rightarrow\left(J^{1} \mathfrak{g}\right)_{a}$ denote the splitting of the exact sequence,

$$
0 \rightarrow T^{*} M \otimes \mathfrak{h} \hookrightarrow\left(J^{1} \mathfrak{g}\right)_{a} \rightarrow \mathfrak{g} \rightarrow 0,
$$

determined by the generator $D$. By the commutativity of diagram 9.2(3) defining $\Theta$, we have

$$
\Theta(X)=\theta(s X) \bmod \operatorname{im} \delta=(s X \cdot d a)^{\vee} \bmod \operatorname{im} \delta .
$$

Invoking (5), we prove (1).

If $\Theta=0$, then $\mathfrak{g}^{(1)}$ is surjective, and so $s(\mathfrak{g}) \subset \mathfrak{g}^{(1)}$ for any generator $D$ of $\mathfrak{g}^{(1)}$; here $s: \mathfrak{g} \rightarrow \mathfrak{g}^{(1)}$ is the corresponding splitting of the exact sequence in (5). This being the case we have, in particular, $s(\mathfrak{g}) \subset\left(J^{1} \mathfrak{g}\right)_{a}$, making $D$ a generator of $\left(J^{1} \mathfrak{g}\right)_{a}$ as well. By (4), $D$ is natural. This proves (2). 
By (44), a natural connection $D$ generates $\mathfrak{g}^{(1)}$ if and only if $(s X \cdot d a)^{\vee}=0$ for all $X \subset \operatorname{ker} \Theta$, where $s$ is the splitting morphism corresponding to $D$. Conclusion (3) is therefore a consequence of (5).

11.2. Prolonging a generator. We now show how to construct a generator for the prolongation $\mathfrak{g}^{(1)}$ of $\mathfrak{g}$ given a generator of $\mathfrak{g}$. Fix a generator $\nabla$ of $\mathfrak{g}$; recall that this is a certain linear connection on $\mathfrak{t}$. Also fix an arbitrary linear connection $\nabla^{\mathfrak{h}}$ on $\mathfrak{h}$. (In practice, there is usually a preferred choice, and if $\mathfrak{t}=T M$ this is always true; see 11.4. No canonical choice exists in general, however.)

With $\nabla$ and $\nabla^{\mathfrak{h}}$ fixed, there is vector bundle morphism

$$
\mathfrak{t} \oplus \mathfrak{h} \stackrel{\tilde{\Theta}}{\rightarrow} \operatorname{Alt}^{2}(T M) \otimes \mathfrak{t}
$$

well defined by its action on sections via

$$
\tilde{\Theta}(V \oplus \phi):=\operatorname{curv} \nabla(\cdot, \cdot) V+d_{\nabla} \phi-\delta\left(\nabla^{\mathfrak{h}} \phi\right) ; \quad V \subset \mathfrak{t}, \phi \subset \mathfrak{h} .
$$

Here

$$
\begin{aligned}
d_{\nabla} \phi\left(U_{1}, U_{2}\right) & :=\nabla_{U_{1}}\left(\phi\left(U_{2}\right)\right)-\nabla_{U_{2}}\left(\phi\left(U_{1}\right)\right)-\phi\left(\left[U_{1}, U_{2}\right]\right) \\
\text { and } \quad\left(\nabla^{\mathfrak{h}} \phi\right) U & :=\nabla_{U}^{\mathfrak{h}} \phi .
\end{aligned}
$$

Theorem (Prolonging a generator of $\mathfrak{g} \subset J^{1} \mathfrak{t}$ ). Let $\mathfrak{g}$ be a surjective infinitesimal geometric structure on a transitive Lie algebroid $\mathfrak{t}$, with $\mathfrak{h}, \nabla, \nabla^{\mathfrak{h}}$, and $\tilde{\Theta}$ as above. Use $\nabla$ to identify $\mathfrak{g}$ with $\mathfrak{t} \oplus \mathfrak{h}$. Then:

(2) The composite morphism,

$$
\mathfrak{g} \cong \mathfrak{t} \oplus \mathfrak{h} \stackrel{\tilde{\Theta}}{\rightarrow} \operatorname{Alt}^{2}(T M) \otimes \mathfrak{t} \stackrel{/ \mathrm{im} \delta}{\longrightarrow} h(\mathfrak{g}),
$$

coincides with the morphism $\Theta: \mathfrak{g} \rightarrow h(\mathfrak{g})$ defined in 9.2 ,

(3) $\operatorname{ker} \Theta \subset \mathfrak{t} \oplus \mathfrak{h}$ is precisely the set of all $V \oplus \phi$ for which the generator equation,

$$
\delta(\epsilon)=\tilde{\Theta}(V \oplus \phi),
$$

admits a solution $\epsilon \in T^{*} M \otimes \mathfrak{h}$.

(4) Assuming $\operatorname{ker} \delta$ and $\operatorname{ker} \Theta$ have constant rank (so that $\mathfrak{g}^{(1)} \subset J^{1} \mathfrak{g}$ is an infinitesimal geometric structure, by Proposition 9.2), a linear connection $\nabla^{(1)}$ on $\mathfrak{g} \cong \mathfrak{t} \oplus \mathfrak{h}$ generating $\mathfrak{g}^{(1)}$ is given by

$$
\nabla_{U}^{(1)}(V \oplus \phi)=\left(\nabla_{U} V+\phi(U)\right) \oplus\left(\nabla_{U}^{\mathfrak{h}} \phi+\epsilon(V \oplus \phi) U\right),
$$

where $\epsilon: \mathfrak{t} \oplus \mathfrak{h} \rightarrow T^{*} M \otimes \mathfrak{h}$ is any of the vector bundle morphisms for which $\epsilon:=\epsilon(V \oplus \phi)$ solves the generator equation defined in (3) above, for each $V \oplus \phi$ in $\operatorname{ker} \Theta$. If $\mathfrak{g}^{(1)}$ is surjective (i.e., $\Theta=0$ ), then every generator is of the above form.

Proof. Let $D$ denote the general form of a natural connection on $\mathfrak{g}$ given in Lemma 11.1(6), with $\epsilon: \mathfrak{t} \oplus \mathfrak{h} \rightarrow T^{*} M \otimes \mathfrak{h}$ completely arbitrary. If $\dot{\Theta}$ is the morphism defined in Proposition 11.1, then one computes

$$
\dot{\Theta}(V \oplus \phi)\left(U_{1}, U_{2}\right)=a\left(\operatorname{curv} D\left(U_{1}, U_{2}\right)(V \oplus \phi)\right)=\tilde{\Theta}(V \oplus \phi)-\delta(\epsilon(V \oplus \phi)),
$$

where $\tilde{\Theta}$ is the morphism defined by (11). Conclusion (2) of the theorem now follows from Proposition 11.1(11). Conclusion (3) is just a consequence of (2). One obtains (41) by taking $\nabla^{(1)}:=D$; choosing $\epsilon$ as described guarantees the curvature condition 
in Proposition 11.1(3). If $\Theta=0$, then every generator of $\mathfrak{g}^{(1)}$ is of the stated form because every generator is natural (Proposition11.1(2)), and all natural connections are included in the general form in Proposition 11.1(6).

11.3. Torsion revisited. The preceding Theorem will allow us to prove Theorem 9.3 relating $\Theta$-reduction and torsion reduction. The appropriate argument also leads to an alternative form of Theorem 11.2 when $\mathfrak{t}=T M$. See 11.4 below.

Although the definition of $\tilde{\Theta}$ in (1) above is explicit, it depends on a choice of linear connection $\nabla^{\mathfrak{h}}$ on $\mathfrak{h}$. Here is an implicit formula depending only on a choice of generator $\nabla$ for $\mathfrak{g}$ :

Proposition. Let $\bar{\nabla}$ denote the $\mathfrak{t}$-connection on $\mathfrak{t}$ associated with a generator $\nabla$ of $\mathfrak{g} \subset J^{1} \mathfrak{t}$. Then for arbitrary sections $X \subset \mathfrak{g}$ and $U_{1}, U_{2} \subset \mathfrak{t}$, one has

$$
\tilde{\Theta}(X)\left(\# U_{1}, \# U_{2}\right)=(X \cdot \operatorname{tor} \bar{\nabla}+\Delta(\operatorname{cocurv} \nabla(a X, \cdot)))\left(U_{1}, U_{2}\right),
$$

where $\Delta$ is the upper coboundary morphism and $a: \mathfrak{g} \rightarrow \mathfrak{t}$ is the projection.

Note that $\operatorname{cocurv} \nabla(a X, \cdot)$ is a section of $T^{*} M \otimes \mathfrak{h}$, by Proposition 6.2)(3).

Proof. Since $\mathfrak{t}$ is assumed to be transitive, there exists a linear connection $\nabla^{\mathfrak{h}}$ on $\mathfrak{h}$ such that $\nabla_{\# U}^{\mathfrak{h}}=\bar{\nabla}_{U}$ for all $U \in \mathfrak{t}$. Here $\bar{\nabla}$ denotes the associated $\mathfrak{t}$-connection on $\mathfrak{h}$ discussed in Example 6.3(3). After a little manipulation, we obtain

$$
\left(d_{\nabla} \phi-\delta\left(\nabla^{\mathfrak{h}} \phi\right)\right)\left(\# U_{1}, \# U_{2}\right)=(\phi \cdot \operatorname{tor} \bar{\nabla})\left(U_{1}, U_{2}\right) ; \quad U_{1}, U_{2} \subset \mathfrak{t} .
$$

Note that $T^{*} M \otimes \mathfrak{t}$ (of which $\phi$ is a section) acts on $\mathrm{Alt}^{2}(\mathfrak{t}) \otimes \mathfrak{t}$ as a subalgebroid of $J^{1} \mathfrak{t}$ (which acts on $\mathfrak{t}$ via adjoint action).

Replace $\mathfrak{g}$ in Proposition 3.8 with $\mathfrak{t}$ and replace $\nabla$ there by the composite

$$
\mathfrak{t} \stackrel{\#}{\longrightarrow} T M \stackrel{\nabla}{\longrightarrow} \mathfrak{g l}(\mathfrak{t}) \text {. }
$$

Then part (2) of that proposition delivers the formula

$$
\begin{aligned}
\operatorname{curv} \nabla\left(\# U_{1}, \# U_{2}\right) V & =\left(\bar{\nabla}_{V} \text { tor } \bar{\nabla}\right)\left(U_{1}, U_{2}\right)-\operatorname{curv} \bar{\nabla}\left(V, U_{1}\right) U_{2} \\
& +\operatorname{curv} \bar{\nabla}\left(V, U_{2}\right) U_{1} ; \quad U_{1}, U_{2}, V \subset \mathfrak{t} .
\end{aligned}
$$

Applying Proposition 4.3(4), we may rewrite this as

$$
\operatorname{curv} \nabla\left(\# U_{1}, \# U_{2}\right) V=\left(\bar{\nabla}_{V} \text { tor } \bar{\nabla}+\Delta(\operatorname{cocurv} \nabla(V, \cdot))\right)\left(U_{1}, U_{2}\right)
$$

Substituting (1) and (2) into the definition 11.2(1) of $\tilde{\Theta}$ gives

$$
\tilde{\Theta}(V \oplus \phi)\left(\# U_{1}, \# U_{2}\right)=\left(\bar{\nabla}_{V} \operatorname{tor} \bar{\nabla}+\phi \cdot \operatorname{tor} \bar{\nabla}+\Delta(\operatorname{cocurv} \nabla(V, \cdot))\right)\left(U_{1}, U_{2}\right) \text {. }
$$

Under the identification $\mathfrak{g} \cong \mathfrak{t} \oplus \mathfrak{h}$ determined by the generator $\nabla$, we obtain the stated formula.

Proof of Theorem 9.3. By Theorem 11.2(2) and the commutativity of 9.1(1), we have

$$
\psi(\Theta(X))=i(\tilde{\Theta}(X)) \bmod \operatorname{im} \Delta,
$$

where $i: \operatorname{Alt}^{2}(T M) \otimes \mathfrak{t} \rightarrow \operatorname{Alt}^{2}(\mathfrak{t}) \otimes \mathfrak{t}$ denotes the natural inclusion $\left(\#^{*}: T^{*} M \rightarrow \mathfrak{t}^{*}\right.$ being injective). The Proposition above then gives

$$
\psi(\Theta(X))=X \cdot \operatorname{tor} \bar{\nabla} \bmod \operatorname{im} \Delta=X \cdot \tau \text {. }
$$


11.4. The special case $\mathfrak{g} \subset J^{1}(T M)$. We now specialize Theorem 11.2 to the case $\mathfrak{t}=T M$. As an application, we complete the proof of Theorem 9.5, the last unproven assertion of the preceding sections.

Let $\mathfrak{g} \subset J^{1}(T M)$ be an infinitesimal geometric structure with structure kernel $\mathfrak{h}$ and generator $\nabla$. Let $\bar{\nabla}$ denote the dual connection, i.e., $\bar{\nabla}_{U} V=\nabla_{V} U+[U, V]$ and define

$$
\begin{gathered}
T M \oplus \mathfrak{h} \stackrel{\tilde{\Theta}}{\rightarrow} \operatorname{Alt}^{2}(T M) \otimes T M, \\
\tilde{\tilde{\Theta}}(V \oplus \phi):=\bar{\nabla}_{V} \text { tor } \bar{\nabla}+\phi \cdot \operatorname{tor} \bar{\nabla}=(V \oplus \phi) \cdot \operatorname{tor} \bar{\nabla} .
\end{gathered}
$$

Theorem (Prolonging a generator of $\mathfrak{g} \subset J^{1}(T M)$ ). With $\mathfrak{g}, \mathfrak{h}, \nabla$, and $\tilde{\tilde{\Theta}}$ as above, we have:

(1) The composite morphism,

$$
\mathfrak{g} \cong T M \oplus \mathfrak{h} \stackrel{\tilde{\Theta}}{\rightarrow} \operatorname{Alt}^{2}(T M) \otimes T M \stackrel{\mathrm{im} \Delta}{\longrightarrow} H(\mathfrak{g}),
$$

coincides with the morphism $\Theta: \mathfrak{g} \rightarrow h(\mathfrak{g})=H(\mathfrak{g})$ defined in 9.2 .

(2) $\operatorname{ker} \Theta \subset \mathfrak{g} \cong T M \oplus \mathfrak{h}$ is precisely the set of all $V \oplus \phi$ for which the generator equation,

$$
\Delta(\epsilon)=\tilde{\tilde{\Theta}}(V \oplus \phi),
$$

admits a solution $\epsilon \in T^{*} M \otimes \mathfrak{h}$.

(3) Assume ker $\Delta$ and $\operatorname{ker} \Theta$ have constant rank so that $\mathfrak{g}^{(1)} \subset J^{1} \mathfrak{g}$ is an infinitesimal geometric structure (by Proposition 9.2 ) and that $H(\mathfrak{g})$ has constant rank (is a $\mathfrak{g}$-representation). Then $\Theta(X)=X \cdot \tau$, where $\tau \subset H(\mathfrak{g})$ is the intrinsic torsion. Also, a linear connection $\nabla^{(1)}$ on $\mathfrak{g} \cong T M \oplus \mathfrak{h}$ generating $\mathfrak{g}^{(1)}$ is given by

$$
\nabla_{U}^{(1)}(V \oplus \phi)=\left(\nabla_{U} V+\phi(U)\right) \oplus\left(\bar{\nabla}_{U} \phi+\epsilon(V \oplus \phi) U+\operatorname{curv} \bar{\nabla}(U, V)\right),
$$

where $\epsilon: T M \oplus \mathfrak{h} \rightarrow T^{*} M \otimes \mathfrak{h}$ is any of the vector bundle morphisms for which $\epsilon:=\epsilon(V \oplus \phi)$ solves the generator equation defined in (2) above, for each $V \oplus \phi$ in $\operatorname{ker} \Theta$. If $\mathfrak{g}^{(1)}$ is surjective (i.e., $\Theta=0$ ), then every generator is of the above form.

Note. The $\epsilon$ 's solving the generator equation above, and the generator equation of Theorem 11.2, are different.

Proof. In 11.2 above take $\mathfrak{t}=T M$ and let $\nabla^{\mathfrak{h}}$ be the $T M$-connection on $\mathfrak{h}$ associated with the generator $\nabla$ (given by 6.2(1) with $\mathfrak{t}=\mathfrak{t}_{1}=T M$ ). Then Proposition 11.3 gives

$$
\tilde{\Theta}(V \oplus \phi)=\tilde{\tilde{\Theta}}(V \oplus \phi)+\Delta(\operatorname{cocurv} \nabla(V, \cdot)) .
$$

Noting that cocurv $\nabla=-\operatorname{curv} \bar{\nabla}$ and $\delta=\Delta$ (because $\mathfrak{t}=T M$ ) one obtains the stated results as a special case of Theorem 11.2 and Theorem 9.3 .

Proof of Theorem 9.5. The hypothesis that $\mathfrak{g}$ be torsion-reduced means $\Theta=0$. So the generator equation defined in (2) above has a solution for all $V \oplus \phi \in$ $T M \oplus \mathfrak{h}$. The solution is unique because $\Delta$ is injective, by hypothesis. Part (1) of 9.5 follows. The Cartan connection on $\mathfrak{g}$ in Theorem 9.5 is the unique generator of $\mathfrak{g}^{(1)}$; conclusion (3) above implies that it has the form given in part (2) of 9.5 . 
Suppose $\mathfrak{g}$ is reductive and let $\nabla$ be a normal generator. Then tor $\bar{\nabla} \subset C$ for some $\mathfrak{g}$-invariant complement $C \subset \operatorname{Alt}^{2}(T M) \otimes T M$ of the image of $\Delta$. In particular,

$$
\bar{\nabla}_{V} \text { tor } \bar{\nabla}+\phi \cdot \operatorname{tor} \bar{\nabla}=(V \oplus \phi) \cdot \operatorname{tor} \bar{\nabla}
$$

is a section of $C$. However, the right-hand side lies in im $\Delta$ because its image under the projection

$$
\operatorname{Alt}^{2}(T M) \otimes T M \stackrel{\text { im } \Delta}{\longrightarrow} H(\mathfrak{g})
$$

is $(V \oplus \phi) \cdot \tau$, which vanishes because $\mathfrak{g}$ is torsion-reduced. We conclude that $\bar{\nabla}_{V}$ tor $\bar{\nabla}+\phi \cdot$ tor $\bar{\nabla}=0$. Part (3i) of Theorem 9.5 follows.

11.5. Symmetries of torsion-free affine structures. We now offer a simple application of Theorem 11.4. Let $\nabla$ be a torsion-free linear connection on $T M$. Then $\nabla$ is a generator of $J^{1}(T M)$. Taking $\mathfrak{g}=J^{1}(T M)$, the generator equation in Theorem 11.4(2) reads $\Delta(\epsilon)=0$, with a trivial solution $\epsilon=0$. Theorem 11.4(3) delivers the following generator $\nabla^{(1)}$ for $J^{2}(T M)=\left(J^{1}(T M)\right)^{(1)}=\mathfrak{g}^{(1)}$ :

$$
\nabla^{(1)}(V \oplus \phi)=\left(\nabla_{U} V+\phi(U)\right) \oplus\left(\nabla_{U} \phi+\operatorname{curv} \nabla(U, V)\right) .
$$

Here we are identifying $\mathfrak{g}=J^{1}(T M)$ with $T M \oplus\left(T^{*} M \otimes T M\right)$ using $\nabla$.

Now let $\mathfrak{g} \subset J^{2}(T M)$ instead denote the isotropy subalgebroid of $\nabla$, as described in 5.8. Then it is not too difficult to check that $\nabla^{(1)}$ is even a generator for $\mathfrak{g}$. In this regard, a helpful formula, readily derived, is

$$
\nabla^{(1)}\left(J^{1} V\right)\left(U_{1}, U_{2}\right)=0 \oplus\left(\left(J^{2} V\right) \cdot \nabla\right)^{\vee}\left(U_{1}, U_{2}\right),
$$

for any section $V \subset T M$.

The generator $\nabla^{(1)}$ is necessarily the Cartan connection on $J^{1}(T M)$ referred to in Proposition 5.8. Its curvature is given by

$$
\operatorname{curv} \nabla^{(1)}\left(U_{1}, U_{2}\right)(V \oplus \phi)=0 \oplus\left(-\left(\nabla_{V} \operatorname{curv} \nabla+\phi \cdot \operatorname{curv} \nabla\right)\left(U_{1}, U_{2}\right)\right) .
$$

In particular, $\operatorname{curv} \nabla^{(1)}$ vanishes if and only if $\operatorname{curv} \nabla$ is $\nabla$-parallel and $T^{*} M \otimes T M$ invariant. But as $\operatorname{id}_{T M}$ is a section of $T^{*} M \otimes T M$, this happens if and only if $\operatorname{curv} \nabla=0$. In that case we obtain

$$
\text { tor } \overline{\nabla^{(1)}}\left(V_{1} \oplus \phi_{1}, V_{2} \oplus \phi_{2}\right)=\left(\phi_{1}\left(V_{2}\right)-\phi_{2}\left(V_{1}\right)\right) \oplus\left[\phi_{1}, \phi_{2}\right]_{T^{*} M \otimes T M},
$$

where $\overline{\nabla^{(1)}}$ denotes the representation of $J^{1}(T M)$ on itself associated with the Car$\tan$ connection $\nabla^{(1)}$ on $J^{1}(T M)$. Applying Theorem 4.6, we recover the following classical result:

Proposition. Let $\nabla$ be a torsion-free linear connection on $T M$ and $\mathfrak{g}_{0}$ the Lie algebra of infinitesimal isometries of $\nabla$ on some open set $\mathcal{U} \subset M$. Then $\operatorname{dim} \mathfrak{g}_{0} \leqslant$ $\operatorname{rank} J^{1}(T M)=n(n+1), n=\operatorname{dim} M$. If $\mathcal{U}$ is simply-connected, then equality holds if and only if curv $\nabla=0$, in which case $\mathfrak{g}_{0}$ is naturally isomorphic to the semidirect product $T_{m} M \oplus\left(T_{m}^{*} M \otimes T_{m} M\right), m \in U$.

\section{Application: Conformal structures}

In this section we turn to the application of Cartan's method to conformal structures. Our results are summarized in Theorems 12.3, 12.4 and 12.6 below. 
12.1. The Lie algebroid setting. Let $\sigma$ be a Riemannian metric on a smooth connected manifold $M$, with $n:=\operatorname{dim} M \geqslant 3$. Let $\langle\sigma\rangle$ denote its conformal class, viewed as the one-dimensional subbundle of $\operatorname{Sym}^{2}(T M)$ generated by the section $\sigma$.

Let $\mathfrak{g}_{\sigma} \subset J^{1}(T M)$ denote the isotropy of $\sigma \subset \operatorname{Sym}^{2}(T M)$ (denoted $\mathfrak{g}$ in [5.2). Let $\mathfrak{g} \subset J^{1}(T M)$ denote the isotropy of $\langle\sigma\rangle \subset \operatorname{Sym}^{2}(T M)$. According to [5.1. this means that the 1-jet of a vector field $V$ at $m \in M$ lies in $\mathfrak{g}$ if and only if $\left(\mathcal{L}_{V} \sigma\right)(m) \in\langle\sigma\rangle(m)$, were $\mathcal{L}$ denotes Lie derivative. Evidently, the symmetries of $\mathfrak{g}$ are the infinitesimal isometries of the conformal structure $\langle\sigma\rangle$, henceforth called the conformal Killing fields.

The rank of $\mathfrak{g}$ is constant (see below), making it an infinitesimal geometric structure on $T M$. Observe that $\mathfrak{g}$ is surjective because $\mathfrak{g}_{\sigma}$ is surjective and $\mathfrak{g}_{\sigma} \subset \mathfrak{g}$. If $\mathfrak{h}_{\sigma}$ and $\mathfrak{h}$ denote the structure kernels of $\mathfrak{g}_{\sigma}$ and $\mathfrak{g}$ respectively, then

$$
\mathfrak{h}=\mathfrak{h}_{\sigma} \oplus\left\langle\operatorname{id}_{T M}\right\rangle \text {, implying } \mathfrak{g}=\mathfrak{g}_{\sigma} \oplus\left\langle\mathrm{id}_{T M}\right\rangle \text {. }
$$

Here $\left\langle\operatorname{id}_{T M}\right\rangle$ denotes the one-dimensional subbundle of $T^{*} M \otimes T M$ generated by the identity section $\operatorname{id}_{T M}$. If vector bundles $E_{1}$ and $E_{2}$ are representations of $\mathfrak{g}$, then a vector bundle morphism $\phi: E_{1} \rightarrow E_{2}$ is a morphism of $\mathfrak{g}$-representations if and only if it is a morphism of $\mathfrak{g}_{\sigma}$-representations commuting with the action of $\operatorname{id}_{T M} \subset \mathfrak{g}$. In particular, this applies to $\alpha \otimes V \mapsto(\alpha \otimes V)^{\mathrm{t}}:=\sigma(V) \otimes \sigma^{-1}(\alpha)$ (the transpose involution of $\left.T^{*} M \otimes T M\right)$ and to the epimorphism, skew : $T^{*} M \otimes T M \rightarrow \mathfrak{h}_{\sigma}$, defined by skew $(\phi):=\left(\phi-\phi^{\mathrm{t}}\right)$. These morphisms and $\mathfrak{h}_{\sigma}$ depend only on the conformal class of $\sigma$.

Because the Levi-Cevita connection $\nabla$ associated with $\sigma$ generates $\mathfrak{g}_{\sigma}$ (see 9.6), it also generates $\mathfrak{g} \supset \mathfrak{g}_{\sigma}$.

12.2. Classical ingredients. From well-known representation-theoretic arguments we know that the curvature of the Levi-Cevita connection $\nabla$ takes values in a proper subbundle $E_{\text {Weyl }} \oplus E_{\text {Ricci }} \subset \operatorname{Alt}^{2}(T M) \otimes \mathfrak{h}_{\sigma}$, with $E_{\text {Ricci }}$ being (for $\operatorname{dim} M \geqslant 3$ ) the isomorphic image of $\operatorname{Sym}^{2}(T M)$ under the monomorphism of $\mathfrak{g}$-representations

$$
\begin{aligned}
T^{*} M \otimes T^{*} M & \stackrel{\text { coRicci }}{\longrightarrow} \operatorname{Alt}^{2}(T M) \otimes \mathfrak{h}_{\sigma}, \\
\operatorname{coRicci}(\Phi)\left(V_{1}, V_{2}\right) & :=\operatorname{skew}\left(\Phi V_{1} \otimes V_{2}-\Phi V_{2} \otimes V_{1}\right) .
\end{aligned}
$$

$E_{\text {Weyl }} \subset \operatorname{Alt}^{2}(T M) \otimes \mathfrak{h}_{\sigma}$ is the intersection of the kernels of the so-called Bianchi and Ricci morphisms; see, e.g., [16, p. 230]. Hence,

$$
\operatorname{curv} \nabla=W+\operatorname{coRicci}(R)
$$

for uniquely determined sections $W \subset E_{\text {Weyl }}$ and $R \subset \operatorname{Sym}^{2}(T M)$. These are the Weyl and modified Ricci curvatures of $\sigma$. Both $E_{\text {Weyl }}$ and $E_{\text {Ricci }}$ are $\mathfrak{g}$-subrepresentations of $\operatorname{Alt}^{2}(T M) \otimes \mathfrak{h}_{\sigma}$, and in particular we may speak of the isotropy $\mathfrak{g}_{W} \subset \mathfrak{g}$ of $W$. If $n=3, E_{\mathrm{Weyl}}=0$.

Also of significance will be the Cotton-York tensor. This is the associated exterior derivative $d_{\nabla} R$ of $R \subset \operatorname{Sym}^{2}(T M) \subset T^{*} M \otimes T^{*} M$, viewed as a $T^{*} M$-value one-form on $M$ :

$$
d_{\nabla} R\left(U_{1}, U_{2}\right):=\nabla_{U_{1}}\left(R\left(U_{2}\right)\right)-\nabla_{U_{2}}\left(R\left(U_{1}\right)\right)-R\left(\left[U_{1}, U_{2}\right]\right) .
$$


Alternatively, by torsion-freeness, $d_{\nabla} R$ is the image of $\nabla R$ under the composite

$$
\begin{gathered}
T^{*} M \otimes \operatorname{Sym}^{2}(T M) \hookrightarrow T^{*} M \otimes T^{*} M \otimes T^{*} M \\
\alpha \operatorname{Alt}^{2}(T M) \otimes T^{*} M, \\
\alpha \otimes \beta \otimes \gamma \mapsto \alpha \wedge \beta \otimes \gamma .
\end{gathered}
$$

Bianchi's second identity 6.5(4) for the generator $\nabla$ enforces a relationship between the Cotton-York tensor $d_{\nabla} R$, and the derivative $\nabla W$. In particular, it is well known that $W=0$ implies the vanishing of $d_{\nabla} R$ in all dimensions except three, where $E_{\mathrm{Weyl}}=0$ and the values of $d_{\nabla} R$ are restricted to a certain six-dimensional subbundle of $\operatorname{Alt}^{2}(T M) \otimes T^{*} M$.

The next two sections summarize the main conclusions of our application of Cartan's method to conformal structures.

\subsection{Conformal invariance of $W$.}

Theorem. The Weyl curvature $W$ is an invariant of $\mathfrak{g}$ and therefore a conformal invariant.

Classically, the conformal invariance of $W$ required an explicit check. In our proof of this theorem conformal invariance is simply a manifestation of the invariance of

the morphism $\Theta$ defined in general in 9.2, as applied to the prolongation of $\mathfrak{g}$ (rather than to $\mathfrak{g}$ itself). This is invariant by construction.

12.4. The $W=0$ case. Our second theorem likewise expresses, in invariant Lie algebroid language, results that are essentially classical:

Theorem. Suppose $W=0$. Then $\mathfrak{g}$ has an associated Cartan algebroid, namely its prolongation $\mathfrak{g}^{(1)} \subset J^{2}(T M)$, which is surjective and transitive. Denoting the Cartan connection on $\mathfrak{g}^{(1)}$ by $\nabla^{(2)}$, we have:

(1) The $\nabla^{(2)}$-parallel sections of $\mathfrak{g}^{(1)} \subset J^{2}(T M)$ are precisely the twice-prolonged conformal Killing fields.

(2) Each metric $\sigma$ in the conformal class determines natural isomorphisms

$$
\mathfrak{g}^{(1)} \cong \mathfrak{g} \oplus T^{*} M, \quad \mathfrak{g} \cong T M \oplus \mathfrak{h},
$$

and an associated explicit formula for $\nabla^{(2)}$ (see 12.11(10) and 12.9(1) below).

(3) If $n \geq 4$, then $\nabla^{(2)}$ is automatically flat. If $n=3$, then $\nabla^{(2)}$ is flat if and only if $d_{\nabla} R=0$. In particular, the Lie algebra $\mathfrak{g}_{0}$ of all conformal Killing fields over any simply-connected open set $\mathcal{U} \subset M$ satisfies

$$
\operatorname{dim} \mathfrak{g}_{0} \leq \operatorname{rank} \mathfrak{g}^{(1)}=\frac{1}{2}(n+1)(n+2),
$$

with equality holding if and only if $n \geq 4$ or $d_{\nabla} R=0$.

12.5. Outline of the application of Cartan's method. Before describing partial results for the general case $W \neq 0$, we sketch the arguments leading to the results above.

Although $\mathfrak{g} \subset J^{1}(T M)$ is surjective, we have $\mathfrak{h} \neq 0$ and Theorem 2.1 does not apply. In 12.7 we show that $\mathfrak{g}$ is already $\Theta$-reduced. The associated lower coboundary morphism is not injective, and Theorem 9.4 is therefore not applicable. 
We turn then, in 12.8 and 12.9, to the prolongation $\mathfrak{g}^{(1)} \subset J^{1} \mathfrak{g}$ of $\mathfrak{g}$. Now $\mathfrak{g}^{(1)}$ is surjective (because $\mathfrak{g}$ is $\Theta$-reduced) but has a non-trivial structure kernel $\mathfrak{h}^{(1)} \cong$ $T^{*} M$.

We show in 12.10 that $\mathfrak{g}^{(1)}$ is already $\Theta$-reduced when $W=0$. The coboundary morphism associated with $\mathfrak{g}^{(1)}$ is injective and Theorem 9.4 applies to $\mathfrak{g}^{(1)}$, making it an associated Cartan algebroid.

12.6. The $W \neq 0$ case and intransitivity. If $W \neq 0$, then $\mathfrak{g}^{(1)}$ is no longer $\Theta$-reduced. According to Proposition 12.10 below, the $\Theta$-reduction of $\mathfrak{g}^{(1)}$ is the preimage of $\mathfrak{g}_{W}$ under the natural projection $\mathfrak{g}^{(1)} \rightarrow \mathfrak{g}$. In particular, this reduction has $\mathfrak{g}_{W} \neq \mathfrak{g}$ as its image and is consequently not surjective. Rather than continue to apply Cartan's method à la 2.6 (turning next to the elementary reduction of the preimage of $\mathfrak{g}_{W}$ ), we remark that $\mathfrak{g}_{W}$ itself is necessarily a reduction of $\mathfrak{g}$ (assuming rank-constancy), and applying the algorithm to $\mathfrak{g}_{W}$ is an easier prospect.

Suppose that $W$ vanishes nowhere. Then the structure kernel of $\mathfrak{g}_{W}$ is contained within that of $\mathfrak{g}_{\sigma}$ (because id $\cdot W=-2 W$ ). Since the upper (= lower) coboundary morphism for $\mathfrak{g}_{\sigma}$ is injective, the same is true for $\mathfrak{g}_{W}$; the prolongation of $\mathfrak{g}_{W}$ (or the prolongation of any reduction of $\mathfrak{g}_{W}$ ) will have a trivial structure kernel. Singularities not withstanding, $\mathfrak{g}$ will therefore have an associated Cartan algebroid that is some reduction of $\mathfrak{g}_{W}$, and in particular will be a subalgebroid of $J^{1}(T M)$ (in contrast to the $W=0$ case). A detailed argument is omitted here.

However we proceed, the following result implies that any associated Cartan algebroid will be intransitive in general: Call $W$ strongly degenerate if there exists a section $\phi \subset \mathfrak{h}$ such that $\nabla_{V} W=\phi \cdot W$, i.e., such that

$$
\begin{aligned}
\left(\nabla_{V} W\right)\left(U_{1}, U_{2}\right) U_{3}=\phi W & \left(U_{1}, U_{2}\right) U_{3} \\
& -W\left(\phi U_{1}, U_{2}\right) U_{3}-W\left(U_{1}, \phi U_{2}\right) U_{3}-W\left(U_{1}, U_{2}\right)\left(\phi U_{3}\right),
\end{aligned}
$$

for all vector fields $V, U_{1}, U_{2}, U_{3}$. We shall see in the proof of the following that this definition is independent of the metric within the conformal class used to fix a Levi-Cevita connection $\nabla$.

Theorem. The isotropy $\mathfrak{g}_{W} \subset J^{1}(T M)$ is surjective (= transitive) if and only if $W$ is strongly degenerate.

The remainder of this section is devoted to proofs of the three preceding Theorems.

12.7. The torsion reduction of $\mathfrak{g}$. Since $\mathfrak{g} \subset J^{1}(T M), \Theta$-reduction is the same as torsion reduction. To compute it, we turn to the upper (= lower) coboundary morphism for $\mathfrak{g}$,

$$
T^{*} M \otimes \mathfrak{h} \stackrel{\Delta}{\rightarrow} \operatorname{Alt}^{2}(T M) \otimes T M .
$$

Its restriction to $T^{*} M \otimes \mathfrak{h}_{\sigma}$ is nothing but the upper coboundary morphism for $\mathfrak{g}_{\sigma}$. Since the latter is an isomorphism (see 9.6) the former is surjective. In particular, $H(\mathfrak{g})=0$, implying $\mathfrak{g}$ is already torsion-reduced. Theorem 9.5 does not apply, however, because $\Delta$ has a non-trivial kernel. Indeed, counting dimensions, we have

$$
\operatorname{rank}(\operatorname{ker} \Delta)=\operatorname{rank}(T M) .
$$


12.8. The first prolongation $\mathfrak{g}^{(1)}$. Since $\mathfrak{g}$ is torsion-reduced (and hence $\Theta$ reduced) the prolongation $\mathfrak{g}^{(1)}$ is surjective (Proposition 2.5) . By Proposition 9.2 , its structure kernel $\mathfrak{h}^{(1)}$ is $\operatorname{ker} \delta=\operatorname{ker} \Delta$. Define a map

$$
\begin{aligned}
T^{*} M & \stackrel{i}{\rightarrow} \operatorname{Sym}^{2}(T M) \otimes T M, \\
i(\alpha) & :=j_{\mathrm{S}}(\alpha)-\sigma \otimes \sigma^{-1}(\alpha),
\end{aligned}
$$

where $j_{\mathrm{S}}: T^{*} M \rightarrow \operatorname{Sym}^{2}(T M) \otimes T M$ is the canonical morphism defined by

$$
j_{\mathrm{S}}(\alpha)\left(V_{1}, V_{2}\right)=\alpha\left(V_{1}\right) V_{2}+\alpha\left(V_{2}\right) V_{1} .
$$

Then $i$ is a monomorphism of $\mathfrak{g}$-representations $(\operatorname{dim} M \geqslant 2)$. Since

$$
i(\alpha) V=\operatorname{skew}(\alpha \otimes V)+\alpha(V) \operatorname{id}_{T M} ; \quad V \subset T M,
$$

we have $i\left(T^{*} M\right) \subset T^{*} M \otimes \mathfrak{h}$. Therefore

$$
i\left(T^{*} M\right) \subset\left(T^{*} M \otimes \mathfrak{h}\right) \cap\left(\operatorname{Sym}^{2}(T M) \otimes T M\right)=\operatorname{ker} \Delta=\mathfrak{h}^{(1)} .
$$

Invoking 12.7(1), we conclude that $\mathfrak{h}^{(1)}=i\left(T^{*} M\right)$.

12.9. A generator for $\mathfrak{g}^{(1)}$. To obtain a generator for $\mathfrak{g}^{(1)}$ we apply Theorem 11.4. Recall that the Levi-Cevita connection $\nabla$ generates $\mathfrak{g}$. We have $\tilde{\tilde{\Theta}}=0$ because $H(\mathfrak{g})=0$. We may therefore take $\epsilon=0$ in 11.4 and, using $\nabla$ to identify $\mathfrak{g}$ with $T M \oplus \mathfrak{h}$, obtain

$$
\nabla_{U}^{(1)}(V \oplus \phi):=\left(\nabla_{U} V+\phi(U)\right) \oplus\left(\nabla_{U} \phi+\operatorname{curv} \nabla(U, V)\right) .
$$

We compute, with the help of Bianchi's second identity,

$$
\operatorname{curv} \nabla^{(1)}\left(U_{1}, U_{2}\right)(V \oplus \phi)=0 \oplus\left(-\left(\nabla_{V} \operatorname{curv} \nabla+\phi \cdot \operatorname{curv} \nabla\right)\left(U_{1}, U_{2}\right)\right) .
$$

This formula may also be written

$$
\operatorname{curv} \nabla^{(1)}\left(U_{1}, U_{2}\right) X=-(X \cdot \operatorname{curv} \nabla)\left(U_{1}, U_{2}\right) \subset \mathfrak{h}_{\sigma} ; \quad X \subset \mathfrak{g} .
$$

12.10. The $\Theta$-reduction of $\mathfrak{g}^{(1)}$. Since $\mathfrak{g}^{(1)} \subset J^{1} \mathfrak{g}$ is surjective and $\mathfrak{g}$ is transitive, the $\Theta$-reduction of $\mathfrak{g}^{(1)}$ is the kernel of a morphism $\mathfrak{g}^{(1)} \rightarrow h\left(\mathfrak{g}^{(1)}\right)$, which we denote by $\Theta^{(1)}$, to distinguish it from the corresponding morphism $\Theta: \mathfrak{g} \rightarrow h(\mathfrak{g})$ for $\mathfrak{g}$; see 9.2. The definition of $h\left(\mathfrak{g}^{(1)}\right)$ depends on the lower coboundary morphism for $\mathfrak{g}^{(1)}$, which we denote by

$$
T^{*} M \otimes \mathfrak{h}^{(1)} \stackrel{\delta^{(1)}}{\longrightarrow} \operatorname{Alt}^{2}(T M) \otimes \mathfrak{g} .
$$

Identifying $\mathfrak{h}^{(1)}$ with $T^{*} M$ as described above, one shows that $\delta^{(1)}$ is the map

$$
\alpha \otimes \beta \mapsto \operatorname{coRicci}(\alpha \otimes \beta)+(\alpha \wedge \beta) \otimes \operatorname{id}_{T M} .
$$

Note that the first term on the right belongs to $\operatorname{Alt}^{2}(T M) \otimes \mathfrak{h}_{\sigma}$ and the second to $\operatorname{Alt}^{2}(T M) \otimes\left\langle\operatorname{id}_{T M}\right\rangle$. In particular, the image of $\delta^{(1)}$ lies entirely within $\operatorname{Alt}^{2}(T M) \otimes$ $\mathfrak{h}$.

Since coRicci is injective $(n \geqslant 3)$ we have $\operatorname{ker} \delta^{(1)}=0$. Therefore the second prolongation $\mathfrak{g}^{(2)}:=\left(\mathfrak{g}^{(1)}\right)^{(1)}$ of $\mathfrak{g}$ has a trivial structure kernel (Proposition 9.2). In particular, $h\left(\mathfrak{g}^{(1)}\right):=\left(\operatorname{Alt}^{2}(T M) \otimes \mathfrak{g}\right) / \operatorname{im} \delta^{(1)}$ has constant rank.

Next, we observe that the composite morphism of $\mathfrak{g}$-representations,

$$
E_{\mathrm{Weyl}} \hookrightarrow \operatorname{Alt}^{2}(T M) \otimes \mathfrak{h}_{\sigma} \hookrightarrow \operatorname{Alt}^{2}(T M) \otimes \mathfrak{g} \stackrel{/ \mathrm{im} \delta^{(1)}}{\longrightarrow} h\left(\mathfrak{g}^{(1)}\right),
$$


is injective. This follows from the description of $\delta^{(1)}$ above and from

$$
\begin{gathered}
E_{\text {Weyl }} \cap E_{\text {Ricci }}=0, \\
\text { where } \quad E_{\text {Ricci }}=\operatorname{coRicci}\left(\operatorname{Sym}^{2}(T M)\right) .
\end{gathered}
$$

Identifying $E_{\text {Weyl }}$ with the corresponding $\mathfrak{g}$-subrepresentation of $h\left(\mathfrak{g}^{(1)}\right)$, we have:

Proposition. The following diagram commutes:

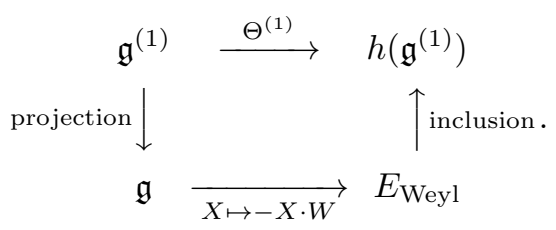

Using the fact that $\mathfrak{g}^{(1)}$ and $\Theta^{(1)}$ are invariants of $\mathfrak{g}$, together with the fact that $\operatorname{id}_{T M}$ is a section of $\mathfrak{g}$, one deduces Theorem 12.3. The proposition also shows that the $\Theta$-reduction of $\mathfrak{g}^{(1)}$ (the kernel of $\Theta^{(1)}$ ) is the preimage of $\mathfrak{g}_{W}$ under the projection $\mathfrak{g}^{(1)} \rightarrow \mathfrak{g}$.

To prove Theorem 12.6. apply Lemma B.1 to the morphism $X \mapsto W \cdot X: \mathfrak{g} \rightarrow$ $E_{\text {Weyl }}$, to show that $\mathfrak{g}_{W}$ has image $D \subset T M$ (a possibly singular distribution on $M)$, where $D$ is the kernel of the morphism

$$
\begin{gathered}
T M \rightarrow E_{\text {Weyl }} /(\mathfrak{h} \cdot W), \\
V \mapsto \nabla_{V} W \bmod \mathfrak{h} \cdot W .
\end{gathered}
$$

Recall here that $\mathfrak{h} \subset T^{*} M \otimes T M$ is the vector bundle whose fiber $\mathfrak{h}(m)$ over $m$ is the Lie algebra of all infinitesimally conformal endomorphisms of $T_{m} M$. This Lie algebra acts on $E_{\text {Weyl }}(m)$ and

$$
\mathfrak{h} \cdot W=\bigcup_{m \in M}\{\phi \cdot W(m) \mid \phi \in \mathfrak{h}(m)\} .
$$

Evidently, $W$ is strongly degenerate if and only if $D=T M$.

Proof of the Proposition. We will apply part (2) of Theorem 11.2, with the roles of $\mathfrak{g}, \mathfrak{t}, \mathfrak{h}, \delta, \Theta, \tilde{\Theta}, \mathfrak{g}^{(1)}$ in the theorem being played by $\mathfrak{g}^{(1)}, \mathfrak{g}, \mathfrak{h}^{(1)}, \delta^{(1)}, \Theta^{(1)}, \tilde{\Theta}^{(1)}, \mathfrak{g}^{(2)}$.

Our first task is to choose a connection $\nabla^{\mathfrak{h}^{(1)}}$ on $\mathfrak{h}$. The Levi-Cevita connection $\nabla$ on $T M$ determines a linear connection on $T^{*} M \otimes\left(T^{*} M \otimes T M\right)$, and one has a chain of inclusions

$$
\mathfrak{h}^{(1)} \subset T^{*} M \otimes \mathfrak{h} \subset T^{*} M \otimes\left(T^{*} M \otimes T M\right),
$$

which we claim are $\nabla$-invariant. The $\nabla$-invariance of $\mathfrak{h} \subset T^{*} M \otimes T M$ follows from Proposition 6.2(2). So the second inclusion is indeed $\nabla$-invariant. Because $\nabla$ generates $\mathfrak{g}$ and because

$$
\Delta: T^{*} M \otimes \mathfrak{h} \rightarrow \operatorname{Alt}^{2}(T M) \otimes T M
$$

is $\mathfrak{g}$-equivariant, it follows that $\Delta$ is $\bar{\nabla}$-equivariant (by Proposition 6.3 ). But $\nabla$ is torsion-free, meaning $\bar{\nabla}$-invariance is the same as $\nabla$-invariance. So the kernel $\mathfrak{h}^{(1)} \subset T^{*} M \otimes \mathfrak{h}$ of $\Delta$ must be $\nabla$-invariant, as claimed. 
We choose $\nabla^{\mathfrak{h}^{(1)}}$ to be the connection that $\mathfrak{h}^{(1)}$ inherits from $T^{*} M \otimes \mathfrak{h}$ as a $\nabla$ invariant subbundle. Appealing to 12.9(1) and the fact that $\mathfrak{h}^{(1)} \subset \operatorname{Sym}^{2}(T M) \otimes$ $T M$, one can show that

$$
\left(d_{\nabla^{(1)}} \phi-\delta^{(1)}\left(\nabla^{\mathfrak{h}^{(1)}} \phi\right)\right)\left(U_{1}, U_{2}\right)=\phi\left(\operatorname{tor} \nabla\left(U_{1}, U_{2}\right)\right)=0,
$$

for all sections $\phi \subset \mathfrak{h}^{(1)} \subset T^{*} M \otimes \mathfrak{h} \subset T^{*} M \otimes \mathfrak{g}$ and $U_{1}, U_{2} \subset T M$.

In the present context 11.2(11) reads

$$
\tilde{\Theta}^{(1)}(X \oplus \phi):=\operatorname{curv} \nabla^{(1)}(\cdot, \cdot) X-d_{\nabla^{(1)}} \phi+\delta^{(1)}\left(\nabla^{\mathfrak{h}^{(1)}} \phi\right) .
$$

From 12.9(2) and (11) above one obtains

$$
\tilde{\Theta}^{(1)}(X \oplus \phi)=-X \cdot \operatorname{curv} \nabla=-X \cdot W+\operatorname{coRicci}(X \cdot R),
$$

for arbitrary sections $X \subset \mathfrak{g}$ and $\phi \subset \mathfrak{h}^{(1)}$. Since $\Theta^{(1)}: \mathfrak{g}^{(1)} \rightarrow h\left(\mathfrak{g}^{(1)}\right)$ is the composite

$$
\mathfrak{g}^{(1)} \cong \mathfrak{g} \oplus \mathfrak{h}^{(1)} \stackrel{\tilde{\Theta}^{(1)}}{\longrightarrow} \operatorname{Alt}^{2}(T M) \otimes \mathfrak{g} \stackrel{/ \mathrm{im} \delta^{(1)}}{\longrightarrow} h\left(\mathfrak{g}^{(1)}\right),
$$

and because the image of $\delta^{(1)}$ contains the image of coRicci, this completes the proof.

12.11. The $W=0$ case. By the above, $\mathfrak{g}^{(1)}$ is $\Theta$-reduced if and only if $\mathfrak{g}_{W}=\mathfrak{g}$. But as $\operatorname{id}_{T M} \subset T^{*} M \otimes T M \subset J^{1}(T M)$ is a section of $\mathfrak{g}$, this is clearly equivalent to the vanishing of $W$.

Assuming $W=0$, Theorem 9.4 applies (with $\mathfrak{g}$ and $\mathfrak{t}$ in the theorem replaced by $\mathfrak{g}^{(1)}$ and $\mathfrak{g}$ ), establishing our claim that $\mathfrak{g}^{(1)}$ is the Cartan algebroid associated with $\mathfrak{g}$ when $W=0$. To compute the Cartan connection $\nabla^{(2)}$ on $\mathfrak{g}^{(1)}$ we will now apply parts (3) and (4) of Theorem 11.2

In the present context, the generator equation defined in Theorem 11.2(3) reads

$$
\delta^{(1)}(\epsilon)+\operatorname{coRicci}(X \cdot R)=0 .
$$

We have used (2) above. Referring to the description of $\delta^{(1)}$ in 12.10, we see that a solution is given by $\epsilon=-X \cdot R$. Using $\nabla^{(1)}$ to identify $\mathfrak{g}^{(1)}$ with $\mathfrak{g} \oplus \mathfrak{h}^{(1)}$, and keeping in mind the identification $\mathfrak{h}^{(1)} \cong T^{*} M$ implicit above, we deduce from Theorem 11.2(4) that

(1) $\nabla_{U}^{(2)}(X \oplus \alpha)=\left(\nabla_{U}^{(1)} X+\operatorname{skew}(\alpha \otimes U)+\alpha(U) \operatorname{id}_{T M}\right) \oplus\left(\nabla_{U} \alpha+(X \cdot R) U\right)$,

for arbitrary sections $X \subset \mathfrak{g}$ and $\alpha \subset T^{*} M$.

We claim

$$
\operatorname{curv} \nabla^{(2)}\left(U_{1}, U_{2}\right)(X \oplus \alpha)=\left(X \cdot d_{\nabla} R\right)\left(U_{1}, U_{2}\right)
$$

where $d_{\nabla} R$ is the Cotton-York tensor, defined in 12.2. In particular, whenever $W=0$, the tensor $d_{\nabla} R$ is a conformal invariant which vanishes if and only if $\nabla^{(2)}$ is flat, i.e., if and only if the Cartan algebroid $\mathfrak{g}^{(1)}$ is flat (Theorem 4.6). This completes the proof of Theorem 12.4 
Proof of (2). Since $W=0$ we have $\operatorname{curv} \nabla=\operatorname{coRicci}(R)$ and, with a little effort, one computes that

$$
\begin{aligned}
\operatorname{curv} \nabla^{(2)}\left(U_{1}, U_{2}\right)(X \oplus \alpha) & =-\left(\left(\nabla_{U_{1}}^{(1)} X\right) \cdot R\right) U_{2}-\nabla_{U_{1}}\left((X \cdot R) U_{2}\right) \\
& +\left(\left(\nabla_{U_{2}}^{(1)} X\right) \cdot R\right) U_{1}+\nabla_{U_{2}}\left((X \cdot R) U_{1}\right) \\
& -(X \cdot R)\left(\left[U_{1}, U_{2}\right]\right) .
\end{aligned}
$$

Equation (2) now follows from the readily verified identities

$$
\begin{gathered}
\left(\nabla_{U}^{(1)} X\right) \cdot V=\nabla_{U}(X \cdot V)-X \cdot\left(\nabla_{U} V\right)+\nabla_{X \cdot U} V \text { for } X \subset \mathfrak{g}, V \subset T M ; \\
\left(\nabla_{U}^{(1)} X\right) \cdot \alpha=\nabla_{U}(X \cdot \alpha)-X \cdot\left(\nabla_{U} \alpha\right)+\nabla_{X \cdot U} \alpha \text { for } X \subset \mathfrak{g}, \alpha \subset T^{*} M .
\end{gathered}
$$

One also makes use of the fact that tor $\nabla=0$.

\section{Appendix A. Cartan groupoids and Lie pseudogroups}

We now explain how flat Cartan algebroids may be viewed as infinitesimal versions of Lie pseudogroups and, conversely, how Lie pseudogroups integrate flat Cartan algebroids. As a byproduct of this discussion, we are led to define Cartan groupoids. These are the global versions of Cartan algebroids and may be viewed as deformations of Lie pseudogroups. Flat Cartan groupoids appear in [19] where they are called 'groupoid etalifications'.

A.1. Lie pseudogroups via pseudoactions. Let us explain, in invariant groupoid language, what it means for a pseudogroup to be a Lie pseudogroup 5 For the classical description see, e.g., [18].

A group of transformations in a smooth manifold $M$ is a Lie group of transformations if it arises from the (smooth) action of some abstract Lie group. Analogously, we declare an arbitrary pseudogroup of transformations in $M$ to be a Lie pseudogroup if it is arises from the pseudoaction of some Lie groupoid. It remains to explain what we mean by pseudoactions and the pseudogroups of transformations they define. We shall understand all constructions to be made in the smooth category.

Let $G$ be a Lie groupoid over $M$. Call an immersed submanifold $\Sigma \subset G$ a pseudotransformation if the restrictions to $\Sigma$ of the groupoid's source and target maps are local diffeomorphisms. In other words, each point of $\Sigma$ should have an open neighborhood in $\Sigma$ that is a (smooth) local bisection of $G$. For example, the pseudotransformations of the pair groupoid $M \times M$ are the local transformations in $M$ taking possibly multiple values.

A pseudoaction of $G$ on $M$ is any foliation $\mathcal{F}$ on $G$ such that:

(1) The leaves of $\mathcal{F}$ are pseudotransformations.

(2) $\mathcal{F}$ is multiplicatively closed.

To define what is meant in (2) let $\hat{\mathcal{F}}$ denote the collection of those subsets of $G$ that are simultaneously an open subset of some leaf of $\mathcal{F}$, and a local bisection. Let $\hat{G}$ denote the collection of all local bisections of $G$, this being a groupoid over the power set of $M$. Then condition (2) is the requirement that $\hat{\mathcal{F}} \subset \hat{G}$ be a subgroupoid.

\footnotetext{
${ }^{5}$ Note added in proof: In fact, what we describe here differs from the classical notion of a Lie pseudogroup. Rather, it is a new class of "differentiable" pseudogroup which will be described further elsewhere.
} 
Given a pseudoaction $\mathcal{F}$ of $G$ on $M$, each element of $\hat{\mathcal{F}}$ defines a local diffeomorphism in $M$ and, by (2), the collection of all such local diffeomorphisms constitutes a pseudogroup of transformations in $M$. For example, if $G$ is an action groupoid $G=G_{0} \times M$, then the canonical horizontal foliation $\mathcal{F}$ furnishes us with the usual pseudogroup of transformations associated with the prescribed action of the Lie group $G_{0}$.

A.2. The flat Cartan algebroid associated with a Lie pseudogroup. Let $\mathcal{G}$ be a Lie pseudogroup of transformations in $M$. Then $\mathcal{G}$ is generated by the pseudoaction $\mathcal{F}$ of some Lie groupoid $G$ over $M$. Define $\hat{\mathcal{F}}$ as in A.1 above. Then each point $g \in G$ lies in some bisection $b \in \hat{\mathcal{F}}$ and all such bisections have the same one-jet at $g$. Thus $\mathcal{F}$ defines a map $D_{\mathcal{F}}: G \rightarrow J^{1} G$ into the Lie groupoid of all one-jets of bisections of $G$. This map, which is a right inverse for the natural projection $J^{1} G \rightarrow G$, is a groupoid morphism because $\mathcal{F}$ is multiplicatively closed.

An arbitrary groupoid morphism $D: G \rightarrow J^{1} G$ furnishing a right inverse for $J^{1} G \rightarrow G$ is what we call a Cartan connection on $G$. These connections may be viewed as certain 'multiplicatively closed' distributions on $G$. The connection $D$ is Frobenius integrable precisely when it comes from a pseudoaction $\mathcal{F}$ as above, in which case $D$ is simply the tangent distribution. A Lie groupoid equipped with a (possibly non-integrable) Cartan connection is a Cartan groupoid. Thus Cartan groupoids are deformed Lie pseudogroups.

Differentiating a Cartan connection $D: G \rightarrow J^{1} G$, we obtain a splitting $\mathfrak{g} \rightarrow J^{1} \mathfrak{g}$ for the exact sequence of Lie algebroids

$$
0 \rightarrow T^{*} M \otimes \mathfrak{g} \hookrightarrow J^{1} \mathfrak{g} \rightarrow \mathfrak{g} \rightarrow 0
$$

This splitting will be a morphism of Lie algebroids, i.e., amounts to a Cartan connection $\nabla$ on $\mathfrak{g}$, as defined in 4.2. If $D=D_{\mathcal{F}}$ for some pseudoaction $\mathcal{F}$ as above, then $D$ is Frobenius integrable and we claim that this implies curv $\nabla=0$, so that $\mathfrak{g}$ is flat (Theorem 4.6); $\mathfrak{g}$ is then a flat Cartan algebroid associated with the pseudogroup $\mathcal{G}$ generated by $\mathcal{F}$.

A.3. The Lie pseudogroup integrating a flat Cartan algebroid. Let $\mathfrak{g}$ be a Cartan algebroid over $M$ with Cartan connection $\nabla$ and assume $\mathfrak{g}$ is the Lie algebroid of some Lie groupoid $G$. For simplicity, suppose $G$ has connected sourcefibers. The connection $\nabla$ determines a Lie algebroid morphism $\mathfrak{g} \rightarrow J^{1} \mathfrak{g}$ splitting the exact sequence of A.2(1). By the groupoid version of Lie's Second Theorem, this morphism integrates to a groupoid morphism $D: G \rightarrow J^{1} G$, i.e., to a Cartan connection on the Lie groupoid $G$. Supposing $\mathfrak{g}$ is flat, we have curv $\nabla=0$ (Theorem 4.6), and we claim this guarantees that $D$ is Frobenius integrable. The integrating foliation $\mathcal{F}$ is a pseudoaction generating a Lie pseudogroup $\mathcal{G}$ of transformations in $M$.

For each locally defined $\nabla$-parallel section $X \subset \mathfrak{g}$, the vector field $\# X \subset T M$ integrates to a one-parameter family of local transformations belonging to $\mathcal{G}$. Conversely each transformation in the pseudogroup $\mathcal{G}$ - or at least each transformation 'close' to the identity — arises as the time-one map associated with such a vector field. In this sense $\mathcal{G}$ integrates the flat Cartan algebroid $\mathfrak{g}$. 


\section{Appendix B. Miscellany}

B.1. On morphisms whose domains sit in a short exact sequence. In the category of vector spaces, or of vector bundles over $M$, let $\theta: B \rightarrow B_{1}$ be an arbitrary morphism, $B_{0}$ its kernel, and suppose $B$ occurs in some exact sequence, as shown below:

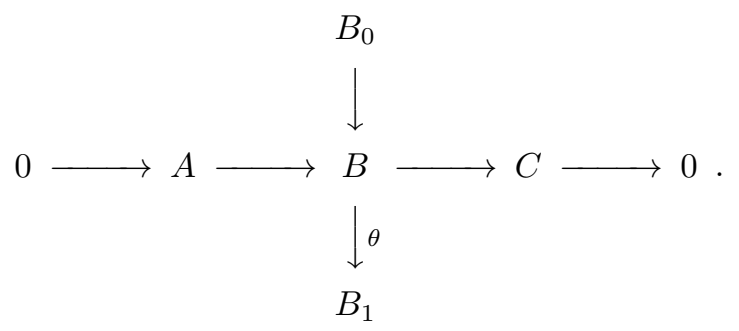

The proof of the following is a straightforward diagram chase.

Lemma. Let $A_{0}$ and $A_{1}$ denote, respectively, the kernel and image of the composite morphism $A \hookrightarrow B \stackrel{\theta}{\rightarrow} B_{1}$. Also, define $C_{1}:=B_{1} / A_{1}$, so that the sequence

$$
0 \rightarrow A_{1} \hookrightarrow B_{1} \rightarrow C_{1} \rightarrow 0
$$

is also exact. Then:

(1) There exists a unique morphism $C \stackrel{\ominus}{\rightarrow} C_{1}$ such that

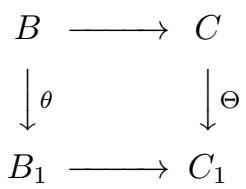

is commutative.

(2) If $C_{0}$ denotes the kernel of $\Theta$, and $B_{0} \hookrightarrow C_{0}$ the restriction of $B \hookrightarrow C$, then the top row in the following commutative diagram is exact (in addition to the other two rows):

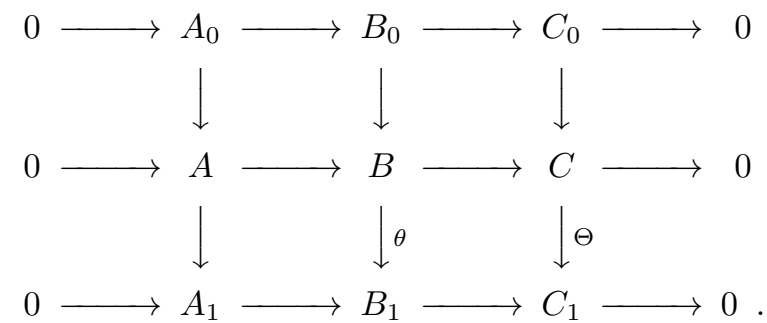

B.2. Gluing Lie algebroid 'point' invariants into global invariants. Let $\mathfrak{g}$ be a transitive Lie algebroid over $M$ and $\mathfrak{h} \subset \mathfrak{g}$ the kernel of its anchor. Let $E$ be a $\mathfrak{g}$-representation. Each fiber $\mathfrak{h}(m)$ of $\mathfrak{h}$ is a Lie algebra acting on the vector space $E(m)$. The following lemma furnishes conditions under which the existence of $\mathfrak{h}(m)$-invariant elements $\sigma(m) \in E(m)$, for each $m \in M$, implies the existence of global $\mathfrak{g}$-invariant sections $\sigma \subset E$. For applications, see 5.2 .

Lemma (Extension Lemma). Suppose that $M$ is simply-connected and that the set $E^{\mathfrak{h}} \subset E$ of $\mathfrak{h}$-invariant elements has constant rank $r>0$. Then $E$ possesses a non-vanishing $\mathfrak{g}$-invariant section $\sigma$. If $r=1$, then $\sigma$ is unique up to constant. 
Proof. Noting that $Y \subset \mathfrak{h}$ implies $[X, Y]_{\mathfrak{g}} \subset \mathfrak{h}$, the identity

$$
Y \cdot(X \cdot \sigma)=X \cdot(Y \cdot \sigma)-[X, Y]_{\mathfrak{g}} \cdot \sigma ; \quad X \subset \mathfrak{g}, Y \subset \mathfrak{h}, \sigma \subset E,
$$

shows that the rank- $r$ subbundle $E^{\mathfrak{h}} \subset E$ is $\mathfrak{g}$-invariant. Because $\mathfrak{h}$ acts trivially on $E^{\mathfrak{h}}$, the representation $\mathfrak{g} \rightarrow \mathfrak{g l}\left(E^{\mathfrak{h}}\right)$ factors through the anchor, delivering a representation $T M \rightarrow \mathfrak{g l}\left(E^{\mathfrak{h}}\right)$, i.e., a flat linear connection $D$ on $E^{\mathfrak{h}}$. One takes $\sigma$ to be any non-vanishing $D$-parallel section of $E^{\mathfrak{h}}$ whose existence is guaranteed by flatness and the simple-connectivity of $M$. The uniqueness claim is clear.

B.3. Proof of Proposition 10.3 , Let $\nabla$ be any generator of $\mathfrak{g}$. Then as $\mathcal{H}, \sigma, \mathbf{n}, \theta$ and $d \theta$ are all $\mathfrak{g}$-invariant, they are all $\bar{\nabla}$-invariant (Proposition 6.3). From the $\bar{\nabla}$-invariance of $\sigma$ and $\mathbf{n}$, one immediately computes

$$
\begin{aligned}
\left(\nabla_{U} \sigma\right)\left(V_{1}, V_{2}\right) & =\sigma\left((\operatorname{tor} \bar{\nabla}(U))_{\mathrm{sym}} V_{1}, V_{2}\right) \\
\text { and } \quad \nabla \mathbf{n} & =\operatorname{tor} \bar{\nabla}(\mathbf{n}),
\end{aligned}
$$

where tor $\bar{\nabla}(U):=\operatorname{tor} \bar{\nabla}(U, \cdot) \subset T^{*} M \otimes T M$ and $U, V_{1}, V_{2}$ are arbitrary vector fields on $M$. Here and in the sequel a subscript sym (or alt) on any type of 2-tensor indicates its symmetrization (resp. skew-symmetrization), defined using the metric $\sigma$ as appropriate. For any 2 -tensor $\phi$, we have $\phi=\left(\phi_{\text {sym }}+\phi_{\text {alt }}\right) / 2$.

From (2) it follows that $\nabla_{\mathbf{n}} \mathbf{n}=0$. From (2) and the $\bar{\nabla}$-invariance of $\theta$, we compute

$$
\theta\left(\nabla_{V} \mathbf{n}\right)=\theta(\operatorname{tor} \bar{\nabla}(\mathbf{n}, V))=d \theta(\mathbf{n}, V)=0 ; \quad V \subset T M .
$$

So $\nabla_{V} \mathbf{n}$ is $\mathcal{H}$-valued, for any $V \subset T M$. This establishes 10.3)(2).

Now $\operatorname{Alt}^{2}(\mathcal{H})$ is rank-one and spanned by $d A$, implying that the restriction of tor $\bar{\nabla}$ to $\mathcal{H}$ (a section of $\operatorname{Alt}^{2}(\mathcal{H})$ ) is of the form $d A \otimes V$, for some vector field $V \subset T M$. However, the $\bar{\nabla}$-invariance of $\theta$ gives

$$
\theta\left(\operatorname{tor} \bar{\nabla}\left(U_{1}, U_{2}\right)\right)=d \theta\left(U_{1}, U_{2}\right)=d A\left(U_{1}, U_{2}\right),
$$

for arbitrary $U_{1}, U_{2} \subset \mathcal{H}$, implying $\theta(V)=1$. We conclude that

$$
(\text { tor } \bar{\nabla}) \mid \mathcal{H}=d A \otimes(\mathbf{b}+\mathbf{n}),
$$

for some unique section $\mathbf{b} \subset \mathcal{H}$. Equation (1) now gives

$$
\left(\nabla_{U} \sigma\right)\left(V_{1}, V_{2}\right)=d A\left(U, V_{1}\right) \sigma\left(\mathbf{b}, V_{2}\right)+d A\left(U, V_{2}\right) \sigma\left(\mathbf{b}, V_{1}\right) ; \quad U, V_{1}, V_{2} \subset \mathcal{H} .
$$

It is not hard to see that this implies

$$
\mathbf{b}=0 \Longleftrightarrow \nabla \sigma \mid \mathcal{H}=0 \text {. }
$$

From (21) and (3) we obtain,

$$
\text { tor } \bar{\nabla}\left(U_{1}+a_{1} \mathbf{n}, U_{2}+a_{2} \mathbf{n}\right)=a_{1} \nabla_{U_{2}} \mathbf{n}-a_{2} \nabla_{U_{1}} \mathbf{n}+d A\left(U_{1}, U_{2}\right)(\mathbf{b}+\mathbf{n}) \text {. }
$$

Therefore, if $\nabla$ is a generator satisfying $\nabla \sigma \mid \mathcal{H}=0$, as in 10.3(3), then 10.3(4) becomes a consequence of (4) above.

If $\nabla \mathbf{n} \subset\left(\mathcal{H}^{*} \otimes \mathcal{H}\right)_{\text {sym }}$, then (2) implies that $(\operatorname{tor} \bar{\nabla}(\mathbf{n}))_{\text {sym }}=\nabla \mathbf{n}$, so that Proposition 10.3 (6) follows from (11) (take $U:=\mathbf{n}$ ).

We return to supposing that $\nabla$ is an arbitrary generator of $\mathfrak{g}$. To establish the remaining claims of the Proposition we require a detailed analysis of the upper coboundary morphism,

$$
T^{*} M \otimes \mathfrak{h} \stackrel{\Delta}{\rightarrow} \operatorname{Alt}^{2}(T M) \otimes T M
$$


By Proposition 10.2, we have $\mathfrak{h} \cong(\mathbb{R} \times M)$, so that $T^{*} M \otimes \mathfrak{h} \cong T^{*} M$. With the help of the $\mathfrak{g}$-invariant splitting $T M=\mathcal{H} \oplus\langle\mathbf{n}\rangle$ and a little multilinear algebra, one identifies a natural isomorphism of $\mathfrak{g}$-representations,

$$
\operatorname{Alt}^{2}(T M) \otimes T M \stackrel{\phi}{\cong} \operatorname{Alt}^{2}(T M) \oplus\left(\mathcal{H}^{*} \otimes \mathcal{H}\right)_{\text {sym }} \oplus \mathcal{H} \oplus(\mathbb{R} \times M),
$$

where $\left(\mathcal{H}^{*} \otimes \mathcal{H}\right)_{\text {sym }} \subset \mathcal{H}^{*} \otimes \mathcal{H}$ denotes the $\mathfrak{g}$-subrepresentation of symmetric elements. We write $\phi=\phi_{1} \oplus \phi_{2} \oplus \phi_{3} \oplus \phi_{4}$ and describe the component morphisms $\phi_{j}$ at the end. Knowing the $\phi_{j}$, one readily establishes the following:

Lemma. Under the identifications above, we have:

(7) The torsion tor $\bar{\nabla} \subset \operatorname{Alt}^{2}(T M) \otimes T M$ is given by

$$
\text { tor } \bar{\nabla}=d \theta \oplus(\nabla \mathbf{n})_{\mathrm{sym}} \oplus \mathbf{b} \oplus f,
$$

where $f \subset(\mathbb{R} \times M)$ is the function on $M$ defined by $(\nabla \mathbf{n})_{\text {alt }} U=f J U$.

(8) The upper coboundary morphism $\Delta$ takes the form

$$
\begin{array}{ccccccccc}
T^{*} M & \stackrel{\Delta}{\longrightarrow} & \operatorname{Alt}^{2}(T M) & \oplus & \left(\mathcal{H}^{*} \otimes \mathcal{H}\right)_{\mathrm{sym}} & \oplus & \mathcal{H} & \oplus & (\mathbb{R} \times M) \\
\alpha & \mapsto & 0 & \oplus & 0 & \oplus & -2 \sigma^{-1}(\alpha) \mid \mathcal{H} & \oplus & \alpha(\mathbf{n})
\end{array}
$$

In particular, $\Delta$ is injective and its image has a complement,

$$
C:=\operatorname{Alt}^{2}(T M) \oplus\left(\mathcal{H}^{*} \oplus \mathcal{H}\right)_{\text {sym }} \oplus 0 \oplus 0,
$$

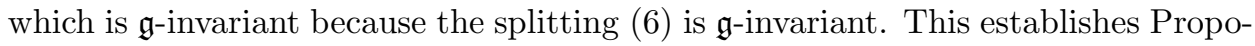
sition 10.3 (1). Also, we obtain $\mathfrak{g}$-invariant isomorphisms,

$$
H(\mathfrak{g}) \cong C \cong \operatorname{Alt}^{2}(T M) \oplus\left(\mathcal{H}^{*} \oplus \mathcal{H}\right)_{\mathrm{sym}} .
$$

This proves the first part of Proposition 10.3 (5).

Now (7) shows that tor $\bar{\nabla} \subset C$ if and only if $\mathbf{b}=0$ and $f=0$. These are true if and only if $\nabla \sigma \mid \mathcal{H}=0$ (by (4)) and $\nabla \mathbf{n} \subset\left(\mathcal{H}^{*} \otimes \mathcal{H}\right)_{\text {sym }}$. This finishes the proof of Proposition 10.3(3).

With $\nabla$ fixed à la Proposition 10.3(3), we have $(\nabla \mathbf{n})_{\text {sym }}=\nabla \mathbf{n}$, and (7) gives

$$
\text { tor } \bar{\nabla}=d \theta \oplus \nabla \mathbf{n} \oplus 0 \oplus 0 .
$$

Under our identifications, the canonical projection $\operatorname{Alt}^{2}(T M) \otimes T M \rightarrow H(\mathfrak{g})$ is just the map

$$
\begin{aligned}
& \operatorname{Alt}^{2}(T M) \oplus\left(\mathcal{H}^{*} \otimes \mathcal{H}\right)_{\mathrm{sym}} \oplus \mathcal{H} \oplus(\mathbb{R} \times M) \rightarrow \mathrm{Alt}^{2}(T M) \oplus\left(\mathcal{H}^{*} \otimes \mathcal{H}\right)_{\mathrm{sym}}, \\
& \lambda \oplus \mu \oplus U \oplus s \mapsto \lambda \oplus \mu .
\end{aligned}
$$

The corresponding map of section spaces sends tor $\bar{\nabla}$ to the intrinsic torsion $\tau$, so that

$$
\tau=d \theta \oplus \nabla \mathbf{n} .
$$

This finishes the proof of 10.3(5) and the Proposition.

The definitions of $\phi_{1}, \phi_{2}, \phi_{3}, \phi_{4}$. The morphism $\phi_{1}$ is the composite morphism

$$
\operatorname{Alt}^{2}(T M) \otimes T M \rightarrow \operatorname{Alt}^{2}(T M) \otimes\langle\mathbf{n}\rangle \cong \operatorname{Alt}^{2}(T M),
$$

where the first arrow is the identity on $\operatorname{Alt}^{2}(T M)$ tensored with the orthogonal projection $T M \rightarrow\langle\mathbf{n}\rangle$. The morphism $\phi_{2}$ is the composite

$$
\operatorname{Alt}^{2}(T M) \otimes T M \rightarrow T^{*} M \otimes T M \rightarrow \mathcal{H}^{*} \otimes \mathcal{H} \rightarrow\left(\mathcal{H}^{*} \otimes \mathcal{H}\right)_{\text {sym }},
$$


where the first arrow is contraction $\rho \mapsto \rho(\mathbf{n}, \cdot)$, the second arrow is obtained by tensoring the restriction $T^{*} M \rightarrow \mathcal{H}^{*}$ with orthogonal projection $T M \rightarrow \mathcal{H}$, and the third arrow is symmetrization. The morphism $\phi_{3}$ is the composite

$$
\operatorname{Alt}^{2}(T M) \otimes T M \rightarrow \operatorname{Alt}^{2}(\mathcal{H}) \otimes \mathcal{H}=\langle d A\rangle \otimes \mathcal{H} \cong \mathcal{H},
$$

where the first arrow is the restriction $\operatorname{Alt}^{2}(T M) \rightarrow \mathrm{Alt}^{2}(\mathcal{H})$ tensored with orthogonal projection $T M \rightarrow \mathcal{H}$. The morphism $\phi_{4}$ is the composite

$$
\operatorname{Alt}^{2}(T M) \otimes T M \rightarrow \operatorname{Alt}^{2}(\mathcal{H}) \otimes\langle\mathbf{n}\rangle=\langle d A\rangle \otimes\langle\mathbf{n}\rangle \cong \mathbb{R} \times M,
$$

where the first arrow is restriction tensored with orthogonal projection.

Relationship with the Levi-Cevita connection. Although we have no need to do so, it is not difficult to express the generator $\nabla$ fixed in Proposition 10.3 (3) in terms of the Levi-Cevita connection $\nabla^{\mathrm{L}-\mathrm{C}}$ associated with $\sigma$ :

$$
\nabla_{U} V=\nabla_{U}^{\mathrm{L}-\mathrm{C}} V-\epsilon(V) U
$$

where $\epsilon \subset T^{*} M \otimes\left(T^{*} M \otimes T M\right)_{\text {alt }}$ is defined by

$$
\begin{aligned}
& \epsilon(\mathbf{n})=\frac{1}{2}\left(\nabla^{\mathrm{L}-\mathrm{C}} \mathbf{n}\right)_{\mathrm{alt}} \\
& \epsilon(U)=\left(\theta \otimes \nabla_{U}^{\mathrm{L}-\mathrm{C}} \mathbf{n}\right)_{\text {alt }} \quad \text { for } U \subset \mathcal{H}, \\
& \text { or } \epsilon(U) V=\left(J \nabla_{U}^{\mathrm{L}-\mathrm{C}} \mathbf{n}\right) \times V \quad \text { for } U \subset \mathcal{H} \text { and } V \subset T M .
\end{aligned}
$$

Here $\times$ denotes cross product and $\left(T^{*} M \otimes T M\right)_{\text {alt }} \subset T^{*} M \otimes T M$ denotes the $\mathfrak{g}$-subrepresentation of skew-symmetric elements.

B.4. On $J^{2} \mathfrak{t}$ as a subbundle of $\left.J^{1}\left(J^{1} \mathfrak{t}\right)\right)$. Here we prove Proposition 8.2 and Lemma 8.1 which describe properties of the second jet bundle $J^{2} \mathfrak{t}$ of an arbitrary vector bundle $t$.

Evidently the formula for $\omega_{1}$ in Proposition 8.2 defines, at the very least, a linear map of section spaces, $\Gamma\left(J^{1}\left(J^{1} \mathfrak{t}\right)\right) \rightarrow \Gamma\left(T^{*} M \otimes J^{1} \mathfrak{t}\right)$. Employing 8.2(1), one shows that $\omega_{1}(f \xi)=f \omega_{1} \xi$ for any smooth function $f$. This implies that the map of section spaces drops to a well defined morphism of vector bundles. A similar argument applies to $\omega_{2}$.

It is not difficult to show that $J^{2} \mathfrak{t} \subset J_{+}^{2} \mathfrak{t}$ and, moreover, that $J^{2} \mathfrak{t} \subset \operatorname{ker} \omega_{2}$. To finish the proof of the proposition it suffices to show that $J^{2} t$ and $\operatorname{ker} \omega_{2}$ have the same rank.

Noting that $J^{1}\left(J^{1} \mathfrak{t}\right)$ sits in an exact sequence,

$$
0 \rightarrow T^{*} M \otimes J^{1} \mathfrak{t} \rightarrow J^{1}\left(J^{1} \mathfrak{t}\right) \rightarrow J^{1} \mathfrak{t} \rightarrow 0,
$$

we apply Lemma B.1 to the morphism $\omega_{1}: J^{1}\left(J^{1} \mathfrak{t}\right) \rightarrow T^{*} M \otimes \mathfrak{t}$ and derive an exact sequence,

$$
0 \rightarrow T^{*} M \otimes T^{*} M \otimes \mathfrak{t} \rightarrow J_{+}^{2} \mathfrak{t} \rightarrow J^{1} \mathfrak{t} \rightarrow 0 .
$$

Next, applying Lemma B.1 to the morphism $\omega_{2}: J_{+}^{2} \mathfrak{t} \rightarrow \operatorname{Alt}^{2}(T M) \otimes \mathfrak{t}$, we derive an exact sequence

$$
0 \rightarrow \operatorname{Sym}^{2}(T M) \otimes \mathfrak{t} \rightarrow \operatorname{ker} \omega_{2} \rightarrow J^{1} \mathfrak{t} \rightarrow 0
$$

Since $J^{2} \mathfrak{t}$ itself occurs in a natural exact sequence

$$
0 \rightarrow \operatorname{Sym}^{2}(T M) \otimes \mathfrak{t} \rightarrow J^{2} \mathfrak{t} \rightarrow J^{1} \mathfrak{t} \rightarrow 0
$$

the bundles $\operatorname{ker} \omega_{2}$ and $J^{2} \mathfrak{t}$ have the same rank. 
Recalling that $X \subset J^{1} \mathfrak{t}$ is holonomic if and only if $\mathcal{D} X=0$, it is not hard to see that $X$ is holonomic if and only if $J^{1} X \subset \operatorname{ker} \omega_{2}$. Lemma 8.1 is then a corollary of Proposition 8.2 .

\section{REFERENCES}

[1] A. D. Blaom. Geometric structures as deformed infinitesimal symmetries. Trans. Amer. Math. Soc., 358:3651-3671, 2006. MR.2218993 (2007g:58023)

[2] R. L. Bryant, S. S. Chern, R. B. Gardner, H. L. Goldschmidt, and P. A. Griffiths. Exterior Differential Systems, volume 18 of Mathematical Sciences Research Institute Publications. Springer-Verlag, New York, 1991. MR 1083148 (92h:58007)

[3] A. Cannas da Silva and A. Weinstein. Geometric Models for Noncommutative Algebras, volume 10 of Berkeley Mathematics Lecture Notes. American Mathematical Society, Providence, RI, 1999. MR1747916 (2001m:58013)

[4] A. Čap and A. R. Gover. Tractor calculi for parabolic geometries. Trans. Amer. Math. Soc., 354(4):1511-1548, 2002. MR1873017 (2003j:53033)

[5] M. Crainic and R. L. Fernandes. Secondary characteristic classes of Lie algebroids. In Quantum Field Theory and Noncommutative Geometry, volume 662 of Lecture Notes in Phys., pages 157-176. Springer, Berlin, 2005. MR2179182(2007b:53047)

[6] Michael Crampin. Cartan connections and Lie algebroids. SIGMA Symmetry Integrability Geom. Methods Appl., 5:Paper 061, 13, 2009. MR2529186

[7] R. L. Fernandes. Lie algebroids, holonomy and characteristic classes. Adv. Math., 170(1):119179, 2002. MR1929305(2004b:58023)

[8] R. B. Gardner. The Method of Equivalence and its Applications. SIAM, Philadelphia, 1989. MR.1062197 (91j:58007)

[9] W. K. Hughen. The sub-Riemannian geometry of three-manifolds. PhD thesis, Duke University, 1995.

[10] T. A. Ivey and J. M. Landsberg. Cartan for Beginners: Differential Geometry via Moving Frames and Exterior Differential Systems, volume 61 of Graduate Studies in Mathematics. American Mathematical Society, 2003. MR2003610 (2004g:53002)

[11] S. Kobayashi. Transformation Groups in Differential Geometry. Springer-Verlag, New York, 1972. MR0355886 (50:8360)

[12] K. C. H. Mackenzie. General Theory of Lie Groupoids and Lie Algebroids, volume 213 of London Mathematical Society Lecture Note Series. Cambridge University Press, Cambridge, 2005. MR2157566 (2006k:58035)

[13] J. E. Marsden and T. S. Ratiu. Introduction to Mechanics and Symmetry, volume 17 of Texts in Applied Mathematics. Springer, 1994. MR1304682 (95i:58073)

[14] R. Montgomery. A tour of subriemannian geometries, their geodesics and applications, volume 91 of Mathematical Surveys and Monographs. American Mathematical Society, 2002. MR $1867362(2002 \mathrm{~m}: 53045)$

[15] P. J. Olver. Equivalence, Invariants, and Symmetry. Cambridge University Press, Cambridge, 1995. MR1337276 (96i:58005)

[16] R. W. Sharpe. Differential Geometry: Cartan's Generalization of Klein's Erlangen Program, volume 166 of Graduate Texts in Mathematics. Springer, 1997. MR1453120 (98m:53033)

[17] S. Sternberg. Lectures on Differential Geometry. AMS Chelsea Publishing, Providence, 1964. MR0193578 (33:1797)

[18] O. Stormark. Lie's Structural Approach to PDE Systems, volume 80 of Encyclopedia of Mathematics and its Applications. Cambridge University Press, Cambridge, 2000. MR 1771254 (2003e:35015)

[19] X. Tang. Deformation Quantization of Pseudo Symplectic (Poisson) Groupoids. Geom. Funct. Anal., 16(3); 731-766, 2006. MR22238946(2007b:53187)

22 Ridge Road, Waiheke Island, New Zealand

E-mail address: anthony.blaom@gmail.com 